\title{
The metabolic syndrome and cardiovascular disease: the CODAM study
}

Citation for published version (APA):

Jacobs, M. (2011). The metabolic syndrome and cardiovascular disease: the CODAM study. [Doctoral Thesis, Maastricht University]. Maastricht University. https://doi.org/10.26481/dis.20110929mj

Document status and date:

Published: 01/01/2011

DOI:

10.26481/dis.20110929mj

Document Version:

Publisher's PDF, also known as Version of record

\section{Please check the document version of this publication:}

- A submitted manuscript is the version of the article upon submission and before peer-review. There can be important differences between the submitted version and the official published version of record.

People interested in the research are advised to contact the author for the final version of the publication, or visit the DOI to the publisher's website.

- The final author version and the galley proof are versions of the publication after peer review.

- The final published version features the final layout of the paper including the volume, issue and page numbers.

Link to publication

\footnotetext{
General rights rights.

- You may freely distribute the URL identifying the publication in the public portal. please follow below link for the End User Agreement:

www.umlib.nl/taverne-license

Take down policy

If you believe that this document breaches copyright please contact us at:

repository@maastrichtuniversity.nl

providing details and we will investigate your claim.
}

Copyright and moral rights for the publications made accessible in the public portal are retained by the authors and/or other copyright owners and it is a condition of accessing publications that users recognise and abide by the legal requirements associated with these

- Users may download and print one copy of any publication from the public portal for the purpose of private study or research.

- You may not further distribute the material or use it for any profit-making activity or commercial gain

If the publication is distributed under the terms of Article $25 \mathrm{fa}$ of the Dutch Copyright Act, indicated by the "Taverne" license above, 
The Metabolic Syndrome and Cardiovascular Disease: The CODAM study 
(C) Marjon Jacobs, 2011

Cover photograph and design by Egon Scheepers

Printed by GVO drukkers \& vormgevers B.V | Ponsen \& Looijen

ISBN: 978-90-6464-497-9 


\title{
The Metabolic Syndrome and Cardiovascular Disease: The CODAM study
}

\author{
PROEFSCHRIFT \\ ter verkrijging van de graad van doctor aan de Universiteit Maastricht, \\ op gezag van de Rector Magnificus, Prof. Mr. G.P.M.F. Mols, \\ volgens het besluit van het College van Decanen, \\ in het openbaar te verdedigen \\ op donderdag 29 september 2011 om 12.00 uur
}

door

Marjon Jacobs 


\section{Promotor}

Prof. Dr. C.D.A. Stehouwer

\section{Copromotores:}

Dr. M.M.J. van Greevenbroek

Dr. C.J.H. van der Kallen

Beoordelingscommissie

Prof. Dr. M.H. Prins (voorzitter)

Prof. Dr. H. ten Cate

Dr. E. Corpeleijn, Universitair Medisch Centrum Groningen, Nederland

Prof. Dr. J.M. Dekker, VU Medisch Centrum Amsterdam, Nederland

Prof. Dr. Ir. A. Hoeks

Esaote Benelux B.V. was gratefully acknowledged for the financial support 


\section{Contents}

Chapter $1 \quad$ General introduction

Chapter 2 Low-grade inflammation can partly explain the association between the metabolic syndrome and either coronary artery disease or severity of peripheral arterial disease: The CODAM study

Chapter 3 The association between the metabolic syndrome and peripheral, but not coronary, artery disease is partly mediated by endothelial dysfunction: The CODAM study

Chapter 4 Human plasma complement C3 is independently associated with coronary heart disease, but only in heavy smokers (the CODAM study)

Chapter 5 The association between the metabolic syndrome and alanine amino transferase is mediated by insulin resistance via related metabolic intermediates (the CODAM study)

Chapter 6 The cross-sectional association between insulin resistance and circulation complement C3 is partly explained by plasma alanine aminotransferase, independent of central obesity and general inflammation (the CODAM study)

Chapter $7 \quad$ Summary and discussion

Samenvatting

Dankwoord

Curriculum Vitae

List of publications

Abbreviations 

Chapter 1

General introduction 



\subsection{Introduction}

Obesity is a chronic disorder affecting over a billion adults world-wide. The prevalence of obesity will probably have doubled by the year 2030 and it is predicted that the obesity epidemic will become a major health problem of the 21 st century [1]. With the increasing number of patients suffering from obesity, the prevalence of complications resulting from the excess of adipose tissue is also growing [2]. Overweight and obesity are associated with high morbidity and mortality, particularly with an increased risk of cardiovascular disease (CVD) and type 2 diabetes mellitus (T2DM) [3-5]. An important common denominator connecting these complications is the metabolic syndrome.

\subsection{The metabolic syndrome}

\subsubsection{Historic overview}

The metabolic syndrome defines a clustering of risk factors that is driven by obesity and the term "metabolic syndrome" dates back to at least the late 1950s, but came into common usage in the late 1970s and described various associations of risk factors with diabetes that had been noted as early as the 1920s [6, 7]. In 1947 Vague observed that upper body obesity appeared to be associated with diabetes and atherosclerosis [8]. Reaven was the first to implicate insulin resistance as the main mechanism underlying the aberrant metabolic changes in the metabolic syndrome [9]. These changes include hyperinsulinemia, hypertension, increased plasma triglyceride concentration, and decreased high-density lipoprotein (HDL) cholesterol concentration, all of which have been shown to be associated with increased risk for CVD as well as T2DM. Since the first description of the metabolic syndrome, initially known as the insulin resistance syndrome and syndrome $\mathrm{X}$, several clinical definitions have been proposed by organisations and study groups (including the World Health Organisation (WHO) [10], International Diabetes Federation (IDF) [11], European Group for study of Insulin Resistance (EGIR) [12] and American Heart Association - National Cholesterol Education Program Adult Treatment Panel III (AHA/NCEP-ATP III) [13]). These different definitions generally included similar components but different emphasis was laid on individual components and different cut-off values were used. Some required presence of specific components like insulin resistance (WHO) or abdominal obesity (IDF) while others (EGIR, AHA / NCEP-ATP III) stated that the presence of 3 out of 5 components means that a patient has the metabolic syndrome. Most of the discussion about the definition of the metabolic syndrome focussed on how 'obesity' should be defined and whether e.g. insulin resistance and microalbuminuria needed to be included. 


\subsubsection{Definition}

The use of different definitions for one and the same syndrome complicated comparability of studies and led to confusion. The definitions of the metabolic syndrome that have been most widely used are from the IDF and the AHA - national heart lung and blood institute (NHLBI). In 2005 they made an attempt to reconcile their clinical definitions but some differences still remained [11, 14]. Recently, the IDF and AHA/NHLBI re-evaluated their definitions and came to the agreement that abdominal obesity should not be a prerequisite for diagnosis but is one of the five criteria and agreed on the definition as presented in Table 1.1 [15]. Further evidence is still needed to determine definitive sex- and ethnicity-specific cut-off values for waist circumference particularly. For this, more longitudinal data on how waist relates to risk of CVD and T2DM are needed in different ethnic populations. Different thresholds have already been defined for various populations (for example: European [16], United states [17], Japanese [18], Chinese [19]). However the question remains whether the same criteria should be applied to someone from a particular ethnic group, regardless of the country they live in [15].

Table 1.1: Criteria for Clinical Diagnosis of the metabolic syndrome[15]

\begin{tabular}{ll}
\hline Measure & Categorical cut-off value \\
\hline Elevated waist circumference & Population- and country-specific definitions \\
& $\geq 102 \mathrm{~cm}$ in males $\#$ \\
& $\geq 88 \mathrm{~cm}$ in females ${ }^{\#}$ \\
\hline Elevated triglycerides & $\geq 1.7 \mathrm{mmol} / \mathrm{L}$ or \\
& drug treatment for elevated triglycerides* \\
\hline Reduced HDL-cholesterol & $<1.03 \mathrm{mmol} / \mathrm{L}$ in males \\
& $<1.3 \mathrm{mmol} / \mathrm{L}$ in females or \\
& drug treatment for reduced HDL-cholesterol* \\
& $\geq 130 \mathrm{mmHg}$ systolic blood pressure and/or \\
& $\geq 85 \mathrm{mmHg}$ diastolic blood pressure or \\
Elevated blood pressure & antihypertensive drug treatment in a patient with a history of hypertension \\
\hline Elevated fasting glucose & $\geq 5.6 \mathrm{mmol} / \mathrm{L}$ or \\
& drug treatment for elevated blood glucose level \\
\hline
\end{tabular}

*the most commonly used drugs for elevated triglycerides and reduced HDL-cholesterol are fibrates and nicotinic acid, subjects taking one of these drugs are presumed to have high triglycerides and/or low HDL-cholesterol; \#these are the values as defined for populations in Europe [16], United States [17], Canada [20] 


\subsubsection{Prevalence of the metabolic syndrome}

In a conglomerate of studies from Sweden, Spain, Netherlands, Italy, France, England and Denmark (in total $>15,000$ subjects), the prevalence of the metabolic syndrome, according to the WHO definition, varied from $13 \%$ in non-diabetic male subjects aged $<40$ yrs, up to $33 \%$ in non-diabetic male subjects older than 55 years of age. For women the prevalence of the metabolic syndrome overall varied from $4 \%$ up to $21 \%$ for the different age-categories [21]. The prevalence of the metabolic syndrome is thus highly age-dependent and, as described in paragraph 2.2, varies between different ethnic populations. It also depends on which definition is used and whether ethnicity specific cut-off values were used. There may be a difference in the prevalence of up to $18 \%$ between the definitions by NCEP-ATP III, IDF, WHO and AHA-NHLBI [22, 23]. But regardless of the exact definition used, the prevalence of the metabolic syndrome is rapidly increasing in developed and in developing countries, worldwide.

\subsubsection{Is the metabolic syndrome a distinct clinical entity?}

There remains debate on the validity of the metabolic syndrome as a distinct entity. It has been argued that the metabolic syndrome falls short of the mark as a predictive tool for CVD risk since there is conflicting evidence on whether the metabolic syndrome provides more information than its components or whether it adds any additional risk to the Framingham Risk Score. Some reported that the metabolic syndrome was associated with CVD risk even after adjustment for conventional risk factors or for the Framingham Risk Score, arguing in favour of its use and relevance [24-26] while others found no evidence for the value of the metabolic syndrome above its components or existing risk models as a predictive tool for CVD and have questioned its utility and recommend against it's use [27-29]. Since the prevalence of (visceral) obesity increased dramatically in the last decades, obesity became important for the prediction of CVD. However, it is not included in the Framingham Risk Score so reconsideration of the Framingham Risk Score would be needed to investigate whether other risk factors like visceral obesity should be included into the score if the metabolic syndrome will not be accepted as a global risk scoring algorithm [30, 31]. Whatever the uncertainties of the definition and aetiology, the metabolic syndrome is a useful measure for early identification of subjects with higher risk of CVD and T2DM.

\subsection{Main metabolic disturbances in the metabolic syndrome}

A disturbed adipose tissue metabolism appears to underlie the metabolic syndrome. This is in the majority of subjects mainly due to excess accumulation of fat (i.e. in obese subjects with the metabolic syndrome) but in a minority of subjects, for instance those who are (genetically) predisposed to a disturbed adipose tissue 
metabolism, it may already be present at lower fat accumulation (i.e. non-obese subjects with the metabolic syndrome). Especially visceral adipose tissue has been identified as an important component of the metabolic syndrome. Metabolically compromised visceral fat lies at the basis of metabolic disturbances in glucose- and lipid metabolism as well as hypertension, possibly via induction of a low-grade inflammatory response and insulin resistance [30]. However, it is not exactly clear in which order these processes occur and several vicious cycles appear to be present (Figure 1.1). The question that remains is how visceral abdominal obesity can lead to inflammation and insulin resistance which will be discussed below.

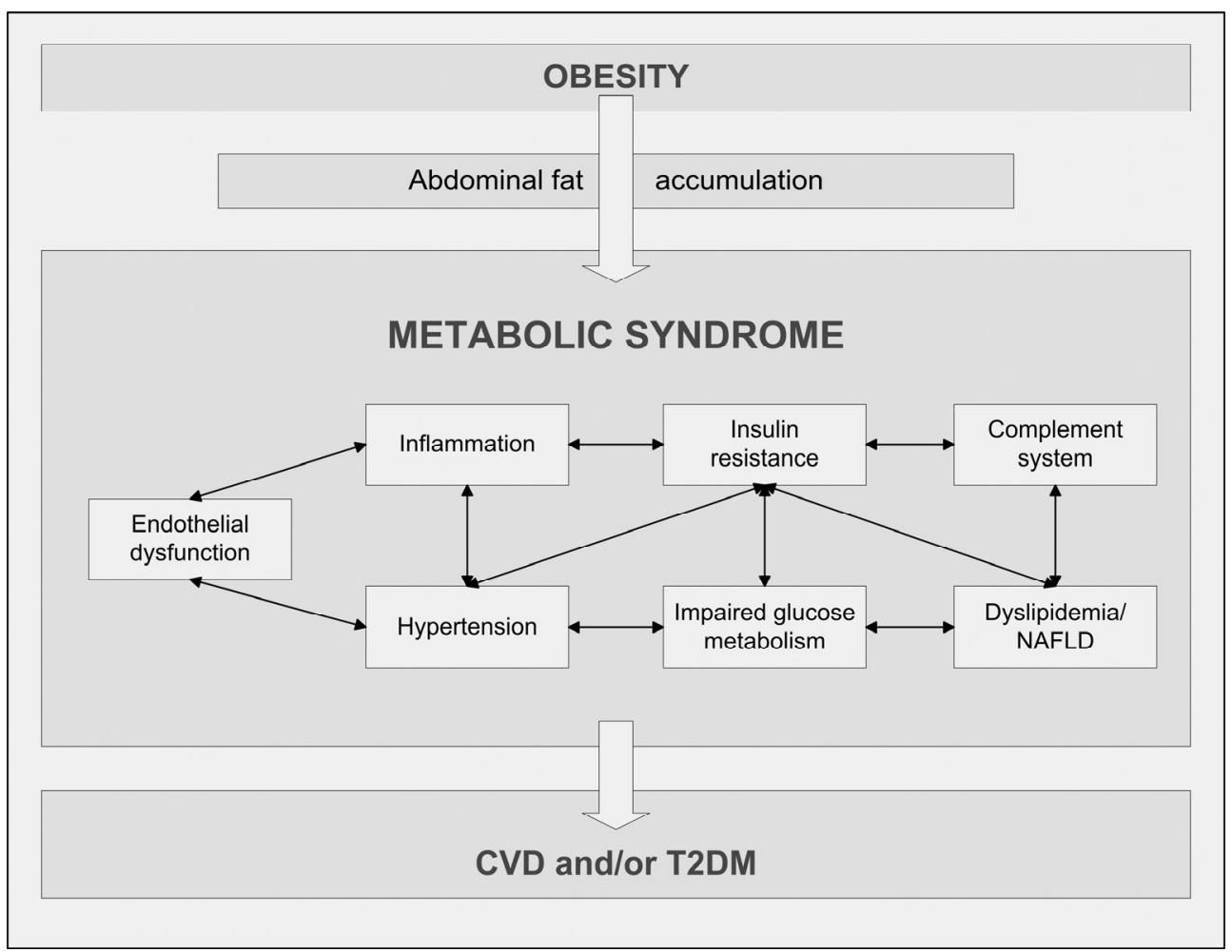

Figure 1.1: The association of obesity with the components of the metabolic syndrome, insulin resistance, inflammation, and endothelial dysfunction which increase the risk of cardiovascular disease and type 2 diabetes

\subsubsection{Inflammation in the metabolic syndrome}

A majority of the subjects with the metabolic syndrome have moderate to substantial abdominal obesity associated with dysregulated visceral adipose tissue metabolism, which is in part a consequence of visceral adipose cell enlargement and the associated 
infiltration of macrophages, leading to adipose-tissue inflammation [30, 32]. Obesity is thus associated with an increased production of inflammatory cytokines, which particularly but not exclusively originate from visceral adipose tissue. These inflammatory cytokines can induce or aggravate insulin resistance in visceral adipose tissue, as well as in other tissues including muscle and liver. Even though considerable insight has been acquired in the mechanisms by which many inflammatory factors mediate insulin resistance, it is still unclear whether inflammation is a primary event that links visceral obesity and insulin resistance, or is rather secondary to hyperglycaemia or hyperlipidaemia.

Tumour necrosis factor $\alpha(\mathrm{TNF} \alpha)$ is one of the first molecular links between obesity and inflammation that was discovered [33, 34]. TNF $\alpha$ activates the intracellular $\mathrm{IKK} \beta / \mathrm{NF} \varkappa \mathrm{B}$ signalling pathway leading to decreased insulin signal transduction via the insulin receptor substrate (IRS). The concept of adipose tissue as a site for the production of cytokines and other bioactive substances quickly extended beyond $\mathrm{TNF} \alpha$ to include interleukin 6 (IL6), IL1 $\beta$, monocyte chemoattractant protein-1 (MCP-1), plasminogen activator inhibitor-1 (PAI-1), angiotensinogen, serum amyloid A (SAA), leptin, resistin, adiponectin and others [35-37]. Many of the proinflammatory cytokines can induce insulin resistance by activating JNK and IKK $\beta / N F \varkappa B$ pathways $[38,39]$. Some adipose tissue-derived factors, e.g. adiponectin and leptin, are true adipokines in the sense that they are produced exclusively by adipocytes, while most of the previously-mentioned cytokines are also expressed in activated macrophages and/or other cell types in the adipose tissue [36]. In contrast to most adipose-derived factors, adiponectin has anti-inflammatory and anti-atherogenic properties [35] and its secretion is reduced in obesity, insulin resistance and T2DM [40]. In the liver, pro-inflammatory gene expression (C-reactive protein (CRP) and others) also increases with increasing adiposity [41]. This suggests that proinflammatory cytokines secreted by visceral adipose tissue induce an inflammatory response in the liver and / or that fat accumulation in the liver, which is a highly prevalent finding in the metabolic syndrome (paragraph 1.3.1), induces an inflammatory response, similar to that in the visceral adipose tissue [36].

The effects of visceral adipose tissue-derived cytokines may be local (paracrine), leading to enhanced insulin resistance in visceral adipose tissue, or systemic (endocrine), thus inducing insulin resistance and other inflammatory responses in other tissues such as muscle and liver. Besides insulin resistance inflammatory cytokines may also induce endothelial dysfunction. TNF $\alpha$ and IL6 can lead to increased expression of adhesion molecules such as intercellular adhesion molecule 1 (ICAM-1) and vascular cell adhesion molecule 1 (VCAM-1) which enhance monocyte adhesion to the endothelium. At the same time MCP-1 does not only increase the migration of monocytes but also favors its transformation into foam cells [42], which 
causes endothelial injury, reflected by higher concentrations of von Willebrand factor (vWf) in plasma [43].

\subsubsection{Complement in the metabolic syndrome}

A specific inflammation- and insulin resistance-associated factor with potential relevance in the metabolic syndrome is complement C3, the most abundant complement protein in the circulation. The complement system is part of the innate immune system and can be activated via three pathways; the classical, the lectin and the alternative pathway [44, 45]. These three pathways all lead to activation of C3, which is mainly produced by the liver but is also expressed by adipose tissue and vascular cells [46, 47] and is strongly associated with obesity and insulin resistance [48, 49] and also with the metabolic syndrome, cardiovascular risk factors and CVD [50, 51].

Complement activation is increasingly recognized as a metabolic regulator in e.g. adipose tissue homeostasis and insulin resistance [52]. It can induce (systemic) inflammation and may specifically be involved in adipose tissue inflammation and insulin resistance $[44,45,52]$.

\subsubsection{Non-esterified fatty acids, non-alcoholic fatty liver disease and dyslipidaemia in the metabolic syndrome}

Loss of insulin sensitivity in adipose tissue can lead to an increased release of nonesterified fatty acids (NEFA) due to decreased inhibition of hormone sensitive lipase by insulin $[53,54]$. Especially visceral adipose tissue drains into the portal circulation and NEFA derived from this particular fat depot will thus be directed towards the liver $[55,56]$, which has several consequences. Firstly, it leads to increased hepatic triglyceride synthesis and increased hepatic concentration of intermediates of NEFA / triglyceride metabolism (e.g. diacylglycerol) that can induce (or worsen) hepatic insulin resistance. Secondly, NEFA stimulate hepatic triglyceride production and this, in combination with insufficient elimination of triglycerides from the liver, contributes to the development of non-alcoholic fatty liver disease (NAFLD) [53, 54]. The combination of hepatic insulin resistance and increased amounts of hepatic triglycerides in NAFLD contributes to an increased production and secretion of type 1 very-low-density lipoproteins (VLDL-1, i.e. the largest and most triglycerideenriched VLDL) rather than VLDL-2 [57]. The most important components of dyslipidemia in the metabolic syndrome include increased plasma triglycerides levels, increased small dense low-density lipoprotein (LDL) cholesterol and decreased levels of HDL cholesterol [58]. This particular type of dyslipidaemia is also known as the atherogenic lipoprotein profile. This atherogenic lipoprotein profile is particularly generated in case of NAFLD, insulin resistance and excess VLDL-1 production. 


\subsection{The metabolic syndrome and cardiovascular disease}

The metabolic syndrome was originally defined as a tool to identify subjects with an elevated risk of CVD. The metabolic syndrome is associated with incident and prevalent CVD in various studies [59]. Inflammation, insulin resistance, dyslipidaemia and complement $\mathrm{C} 3$, as described in the previous paragraphs in relation to the metabolic syndrome, are all also associated with a higher risk of CVD [51, 60-63].

Inflammatory cytokines enhance the attachment to and migration of monocytes into the vessel wall and their conversion to macrophages [64]. This process is integral to the development of atherosclerosis. Macrophages and smooth muscle cells in the arterial wall will accumulate cholesterol-rich lipoproteins (LDL and small dense LDL), which results in the formation of lipid-laden foam cells and fatty streaks. Ultimately, these early lesions will evolve into advanced atherosclerotic plaques. Especially TNF $\alpha$ is involved in this process. It activates the transcription factor NF- $x \mathrm{~B}$, which orchestrates a series of inflammatory changes in the vascular tissue, including expression of adhesion molecules, like ICAM-1 and VCAM-1 [65]. The cytokine IL-6 stimulates hepatic production of CRP, which comprises an important marker of vascular inflammation and is a predictor of atherosclerosis [66-68].

Insulin resistance leads to generation of the atherogenic lipoprotein profile which can in turn accelerate the atherosclerotic process. Firstly, because specific properties of the small dense LDL make them even more atherogenic than normal LDL. On the one hand because of their size, they penetrate the arterial wall more easily, and on the other hand since they are more easily oxidized, small dense LDL forms a better substrate for uptake in macrophages. Secondly, because the lower levels of HDL concomitantly compromise reverse cholesterol transport. Insulin resistance may also lead to hyperinsulinaemia, (relative) $\beta$-cell exhaustin and subsequently to hyperglycaemia which might additionally accelerate the atherosclerotic process. Several mechanisms via which this might occur have been described. One of the mechanisms is that non-enzymatic glycosylation of (lipo-)proteins in the arterial wall changes the normal function of proteins that might lead to interference with receptor recognition and changing enzymatic activity. Another proposed mechanism is the activation of protein kinase $\mathrm{C}$ (PKC) after exposure to high glucose, resulting in an increase of transforming growth factor- $\beta$ expression. This factor plays an important role in regulating extracellular matrix production by activating gene expression of proteoglycans and collagen [69]. These describe hyperglycemia-related phenomena that may be most relevant in subjects with metabolic syndrome-associated T2DM, rather than in the metabolic syndrome in general.

Of note, CVD has various aspects with different aetiology including among others coronary heart disease (CHD) and peripheral arterial disease (PAD). CHD occurs in the coronary vascular bed while PAD and stroke occur in the peripheral and cerebral vascular bed, respectively. CHD is the most frequent vascular disease associated with 
the metabolic syndrome and occurs when the elastic smooth vessel wall of coronary arteries develop plaques, including fatty deposits and inflammatory cells. This leads to obstruction of the arteries causing ischaemia. The plaques might eventually rupture which causes total occlusion of the coronary artery and acute coronary artery disease. The vessels most affected by PAD are large arteries, the aorta and arteries of the lower extremities. More than $90 \%$ of the atherosclerotic disease results in arterial problems in the lower extremities [70]. Also in these vessels atherosclerosis plays an important role in the hardening and narrowing of the arteries. The epidemiology, prognosis, pathophysiology, treatment, and prevention of PAD in relation to the metabolic syndrome have been less studied than the association between the metabolic syndrome and CHD. Since CHD and PAD occur in different vascular beds they might be affected in a different way by the metabolic syndrome. In addition, the exact pathways how the metabolic syndrome leads to CVD are currently unknown.

\subsection{Study population \& Outline of this thesis}

The association between the metabolic syndrome and CVD and possible mechanisms were described in the previous paragraphs. In this thesis, we focused on the specific processes (inflammation, endothelial dysfunction, insulin resistance, dyslipidaemia and the complement system) that may play a role in linking the metabolic syndrome to CVD, CHD and PAD.

\subsubsection{Study population}

All studies described in this thesis were performed in the baseline measurements of the Cohort on Diabetes and Atherosclerosis Maastricht (CODAM).

The CODAM study (started in 1999) consists of subjects with an increased risk of T2DM and CVD [71-73]. In short, participants of a large ongoing cohort of the National Institute for Public Health and the Environment who were Caucasian, older than 40 years of age and had one or more risk factors (Table 1.2) were invited to participate in a screening for the CODAM study. During the screening phase all participants $(n=2715)$ underwent an oral glucose tolerance test (OGTT, with capillary glucose measurements at $\mathrm{t}=0$ and $\mathrm{t}=120$ minutes). According to the $1999 \mathrm{WHO}$ criteria [74], $226(8.3 \%)$ were newly diagnosed with T2DM, $385(14.2 \%)$ had impaired glucose tolerance (IGT), 215 (7.9\%) had impaired fasting glucose (IFG) and 1889 $(69.6 \%)$ had normal glucose tolerance (NGT). All those screened with T2DM, a part of those screened with IGT/IFG and a random selection with NGT, were invited to participate in CODAM. To increase the power of the participants with T2DM 49 participants who were known to have T2DM of recent onset were also invited. Eventually a total of 574 subjects were included in the CODAM study. Based on a second OGTT (with venous glucose measurements at $\mathrm{t}=0,30,60$ and 120 minutes), 
301 had NGT, 127 had impaired glucose metabolism (IGM; IGT and IFG combined) and 146 had T2DM (of whom 80 were newly diagnosed by screening, 20 had T2DM of recent onset, i.e. diabetes more than 1 year but less than 4 years and 46 had T2DM for 4 years or longer.

Table 1.2: Inclusion criteria CODAM study

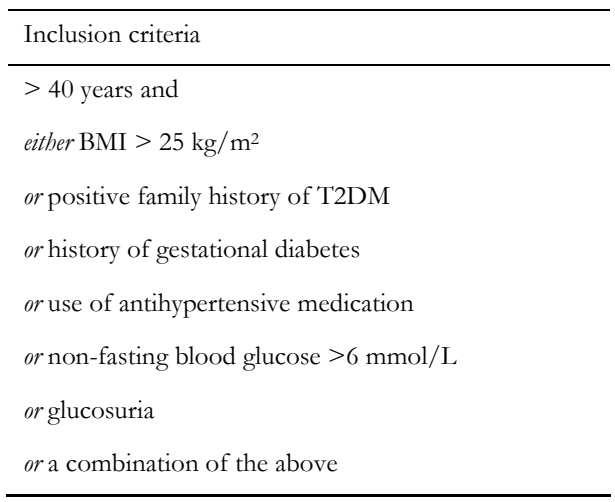

All participants visited the laboratory twice. During the first visit, the participants handed in questionnaires on lifestyle factors and health status. Body weight and blood pressure were measured, and the 2nd OGTT was done. Blood samples were taken to determine various blood parameters including blood lipids, insulin, markers of inflammation and endothelial dysfunction. During the OGT'T several questionnaires were filled in (about smoking habits, physical activity, WHO Rose angina questionnaire, health related quality of live, depression, medication, and food frequency questionnaire). During the second visit, blood pressure was measured again, carotid artery wall ultrasound measurements were taken as a surrogate marker of atherosclerosis, and ankle arm index (AAIx) was measured as a marker of peripheral arterial disease. Next, body height, body composition (skin fold thickness) and body fat distribution (waist and hip circumference, saggital and transversal diameter) were measured and a 12-lead rest electrocardiogram (ECG) was recorded.

In the course of this $\mathrm{PhD}$ project, a 7 years follow-up examination has been completed but during preparation of this thesis, the data for longitudinal investigations were not yet available.

\subsubsection{Outline}

The central aim of this thesis was to identify pathways that can (partly) explain the association between the metabolic syndrome and cardiovascular disease. The analyses were done in a population with an elevated risk of T2DM (the CODAM study). 
We investigated how different aspects of the metabolic syndrome are related to CVD and CVD risk associates. In Chapter $2 \& 3$ we investigated the association between the metabolic syndrome and two different aspects of CVD, i.e. coronary artery disease and peripheral arterial disease. In Chapter 2 we determined whether plasma markers of low-grade inflammation could explain, at least partly, the association between the metabolic syndrome and CVD. This was expanded in Chapter 3 by also investigating if endothelial dysfunction could also explain part of the association and whether it was additional to low-grade inflammation. In Chapter 4 we investigated whether complement $\mathrm{C} 3$ was associated with CHD.

In Chapter 5 we focussed on the association between the metabolic syndrome and structural and metabolic intermediates of CVD. We report on the association of the metabolic syndrome with alanine-amino transferase as a marker of NAFLD which, in turn, is a risk factor for CVD. We determined if the association was explained by possible links between the metabolic syndrome and CVD, i.e. insulin resistance, lowgrade inflammation, endothelial dysfunction and NEFAs. In Chapter 6 we report on potential mechanisms relating human plasma complement C3 to insulin resistance. Finally, Chapter 7 summarizes and discusses the main findings of the studies included in this thesis and their implications in public health.

\subsection{References}

1 Zimmet P, Alberti KG, Shaw J. Global and societal implications of the diabetes epidemic. Nature 2001;414(6865):782-787.

2 Ezzati M, Lopez AD, Rodgers A, et al. Comparative quantification of health risks: global and regional burden of disease attribution to selected major risk factors. Geneva; 2004.

3 Casanueva FF, Moreno B, Rodriguez-Azeredo R, et al. Relationship of abdominal obesity with cardiovascular disease, diabetes and hyperlipidaemia in Spain. Clin Endocrinol (Oxf) 2009;

4 Caterson ID, Hubbard V, Bray GA, et al. Prevention Conference VII: Obesity, a worldwide epidemic related to heart disease and stroke: Group III: worldwide comorbidities of obesity. Circulation 2004;110(18):e476-483.

5 Yusuf S, Hawken S, Ounpuu S, et al. Obesity and the risk of myocardial infarction in 27,000 participants from 52 countries: a case-control study. Lancet 2005;366(9497):1640-1649.

6 Joslin EP. The prevention of diabetes mellitus. JAMA 1921;76(2):79-84.

$7 \quad$ Kylin E. Studies of the hypertension-hyperglycemia-hyperuricemia syndrome. Zentralbl Inn Med 1923;44(105-127.

8 Vague J. La diffférenciacion sexuelle, facteur déterminant des formes de l'obésité. Presse Med 1947;55(30):339.

9 Reaven GM. Banting lecture 1988. Role of insulin resistance in human disease. Diabetes 1988;37(12):1595-1607. 
Alberti KG, Zimmet PZ. Definition, diagnosis and classification of diabetes mellitus and its complications. Part 1: diagnosis and classification of diabetes mellitus provisional report of a WHO consultation. Diabet Med 1998;15(7):539-553.

1 Alberti KG, Zimmet P, Shaw J. The metabolic syndrome--a new worldwide definition. Lancet 2005;366(9491):1059-1062.

2 Balkau B, Charles MA. Comment on the provisional report from the WHO consultation. European Group for the Study of Insulin Resistance (EGIR). Diabet Med 1999;16(5):442-443.

Executive Summary of The Third Report of The National Cholesterol Education Program (NCEP) Expert Panel on Detection, Evaluation, And Treatment of High Blood Cholesterol In Adults (Adult Treatment Panel III). Jama 2001;285(19):2486-2497.

Grundy SM, Cleeman JI, Daniels SR, et al. Diagnosis and management of the metabolic syndrome: an American Heart Association/National Heart, Lung, and Blood Institute Scientific Statement. Circulation 2005;112(17):2735-2752.

5 Alberti KG, Eckel RH, Grundy SM, et al. Harmonizing the metabolic syndrome: a joint interim statement of the International Diabetes Federation Task Force on Epidemiology and Prevention; National Heart, Lung, and Blood Institute; American Heart Association; World Heart Federation; International Atherosclerosis Society; and International Association for the Study of Obesity. Circulation 2009;120(16):1640-1645.

6 Graham I, Atar D, Borch-Johnsen K, et al. European guidelines on cardiovascular disease prevention in clinical practice: executive summary. Atherosclerosis 2007;194(1):1-45.

Clinical Guidelines on the Identification, Evaluation, and Treatment of Overweight and Obesity in Adults--The Evidence Report. National Institutes of Health. Obes Res 1998;6 Suppl 2(51S-209S.

Oka R, Kobayashi J, Yagi K, et al. Reassessment of the cutoff values of waist circumference and visceral fat area for identifying Japanese subjects at risk for the metabolic syndrome. Diabetes Res Clin Pract 2008;79(3):474-481.

New criteria for 'obesity disease' in Japan. Circ J 2002;66(11):987-992.

Khan NA, McAlister FA, Rabkin SW, et al. The 2006 Canadian Hypertension Education Program recommendations for the management of hypertension: Part II - Therapy. Can J Cardiol 2006;22(7):583-593.

1 Balkau B, Charles MA, Drivsholm T, et al. Frequency of the WHO metabolic syndrome in European cohorts, and an alternative definition of an insulin resistance syndrome. Diabetes Metab 2002;28(5):364-376.

2 Ghosh A, Liu T, Khoury MJ, et al. Family history of diabetes and prevalence of the metabolic syndrome in U.S. adults without diabetes: 6-year results from the National Health and Nutrition Examination Survey (1999-2004). Public Health Genomics 13(6):353-359.

3 Welin L, Adlerberth A, Caidahl K, et al. Prevalence of cardiovascular risk factors and the metabolic syndrome in middle-aged men and women in Gothenburg, Sweden. BMC Public Health $2008 ; 8(403$. 

events in the Scandinavian Simvastatin Survival Study (4S) and the Air Force/Texas Coronary Atherosclerosis Prevention Study (AFCAPS/TexCAPS). Am J Cardiol 2004;93(2):136-141. Malik S, Wong ND, Franklin SS, et al. Impact of the metabolic syndrome on mortality from coronary heart disease, cardiovascular disease, and all causes in United States adults. Circulation 2004;110(10):1245-1250.

26 Sattar N, Gaw A, Scherbakova O, et al. Metabolic syndrome with and without C-reactive protein as a predictor of coronary heart disease and diabetes in the West of Scotland Coronary Prevention Study. Circulation 2003;108(4):414-419.

Bruno G, Merletti F, Biggeri A, et al. Metabolic syndrome as a predictor of all-cause and cardiovascular mortality in type 2 diabetes: the Casale Monferrato Study. Diabetes Care 2004;27(11):2689-2694.

Kahn R, Buse J, Ferrannini E, et al. The metabolic syndrome: time for a critical appraisal: joint statement from the American Diabetes Association and the European Association for the Study of Diabetes. Diabetes Care 2005;28(9):2289-2304.

Stern MP, Williams K, Gonzalez-Villalpando C, et al. Does the metabolic syndrome improve identification of individuals at risk of type 2 diabetes and/or cardiovascular disease? Diabetes Care 2004;27(11):2676-2681.

Arsenault BJ, Pibarot P, Despres JP. The quest for the optimal assessment of global cardiovascular risk: are traditional risk factors and metabolic syndrome partners in crime? Cardiology 2009;113(1):35-49. Lau DC. Metabolic syndrome: perception or reality? Curr Atheroscler Rep 2009;11(4):264-271. Gustafson B, Gogg S, Hedjazifar S, et al. Inflammation and impaired adipogenesis in hypertrophic obesity in man. Am J Physiol Endocrinol Metab 2009; Hotamisligil GS, Arner P, Caro JF, et al. Increased adipose tissue expression of tumor necrosis factor-alpha in human obesity and insulin resistance. J Clin Invest 1995;95(5):2409-2415. Kern PA, Saghizadeh M, Ong JM, et al. The expression of tumor necrosis factor in human adipose tissue. Regulation by obesity, weight loss, and relationship to lipoprotein lipase. J Clin Invest 1995;95(5):2111-2119. Gustafson B, Hammarstedt A, Andersson CX, et al. Inflamed adipose tissue: a culprit underlying the metabolic syndrome and atherosclerosis. Arterioscler Thromb Vasc Biol 2007;27(11):2276-2283.

36 Shoelson SE, Lee J, Goldfine AB. Inflammation and insulin resistance. J Clin Invest 2006;116(7):1793-1801.

37 Wellen KE, Hotamisligil GS. Inflammation, stress, and diabetes. J Clin Invest 2005;115(5):1111-1119.

38 Hirosumi J, Tuncman G, Chang L, et al. A central role for JNK in obesity and insulin resistance. Nature 2002;420(6913):333-336.

39 Werner ED, Lee J, Hansen L, et al. Insulin resistance due to phosphorylation of insulin receptor substrate-1 at serine 302. J Biol Chem 2004;279(34):35298-35305. 

Jansson PA, Pellme F, Hammarstedt A, et al. A novel cellular marker of insulin resistance and early atherosclerosis in humans is related to impaired fat cell differentiation and low adiponectin. Faseb J 2003;17(11):1434-1440.

Cai D, Yuan M, Frantz DF, et al. Local and systemic insulin resistance resulting from hepatic activation of IKK-beta and NF-kappaB. Nat Med 2005;11(2):183-190.

2 Wajchenberg BL, Nery M, Cunha MR, et al. Adipose tissue at the crossroads in the development of the metabolic syndrome, inflammation and atherosclerosis. Arq Bras Endocrinol Metabol 2009;53(2):145-150.

Goldberg RB. Cytokine and cytokine-like inflammation markers, endothelial dysfunction, and imbalanced coagulation in development of diabetes and its complications. J Clin Endocrinol Metab 2009;94(9):3171-3182.

Walport MJ. Complement. First of two parts. N Engl J Med 2001;344(14):1058-1066.

Walport MJ. Complement. Second of two parts. N Engl J Med 2001;344(15):1140-1144.

Gabrielsson BG, Johansson JM, Lonn M, et al. High expression of complement components in omental adipose tissue in obese men. Obes Res 2003;11(6):699-708.

7 Hoie EB, McGuire TR, Leuschen PM, et al. Pentoxifylline inhibits tumor necrosis factor-alpha induced synthesis of complement component C3 in human endothelial cells. Biol Pharm Bull 2004;27(10):1670-1673.

Muscari A, Antonelli S, Bianchi G, et al. Serum C3 is a stronger inflammatory marker of insulin resistance than C-reactive protein, leukocyte count, and erythrocyte sedimentation rate: comparison study in an elderly population. Diabetes Care 2007;30(9):2362-2368.

Weyer C, Tataranni PA, Pratley RE. Insulin action and insulinemia are closely related to the fasting complement C3, but not acylation stimulating protein concentration. Diabetes Care 2000;23(6):779-785.

Engstrom G, Hedblad B, Janzon L, et al. Complement C3 and C4 in plasma and incidence of myocardial infarction and stroke: a population-based cohort study. Eur J Cardiovasc Prev Rehabil 2007;14(3):392-397.

Muscari A, Bozzoli C, Puddu GM, et al. Association of serum C3 levels with the risk of myocardial infarction. Am J Med 1995;98(4):357-364.

2 Mamane Y, Chung Chan C, Lavallee G, et al. The C3a anaphylatoxin receptor is a key mediator of insulin resistance and functions by modulating adipose tissue macrophage infiltration and activation. Diabetes 2009;58(9):2006-2017.

3 Griffin ME, Marcucci MJ, Cline GW, et al. Free fatty acid-induced insulin resistance is associated with activation of protein kinase $\mathrm{C}$ theta and alterations in the insulin signaling cascade. Diabetes 1999;48(6):1270-1274.

4 Kim JK, Gimeno RE, Higashimori T, et al. Inactivation of fatty acid transport protein 1 prevents fat-induced insulin resistance in skeletal muscle. J Clin Invest 2004;113(5):756-763.

5 Donnelly KL, Smith CI, Schwarzenberg SJ, et al. Sources of fatty acids stored in liver and secreted via lipoproteins in patients with nonalcoholic fatty liver disease. J Clin Invest 2005;115(5):1343-1351. 
Puri P, Baillie RA, Wiest MM, et al. A lipidomic analysis of nonalcoholic fatty liver disease. Hepatology 2007;46(4):1081-1090.

57 Lewis GF. Fatty acid regulation of very low density lipoprotein production. Curr Opin Lipidol 1997;8(3):146-153.

Grundy SM. Hypertriglyceridemia, atherogenic dyslipidemia, and the metabolic syndrome. Am J Cardiol 1998;81(4A):18B-25B.

Mottillo S, Filion KB, Genest J, et al. The metabolic syndrome and cardiovascular risk a systematic review and meta-analysis. J Am Coll Cardiol 2010;56(14):1113-1132.

Jager A, van Hinsbergh VW, Kostense PJ, et al. von Willebrand factor, C-reactive protein, and 5-year mortality in diabetic and nondiabetic subjects: the Hoorn Study. Arterioscler Thromb Vasc Biol 1999;19(12):3071-3078.

1 Langenberg C, Bergstrom J, Scheidt-Nave C, et al. Cardiovascular death and the metabolic syndrome: role of adiposity-signaling hormones and inflammatory markers. Diabetes Care 2006;29(6):1363-1369.

Schindhelm RK, Dekker JM, Nijpels G, et al. Alanine aminotransferase predicts coronary heart disease events: a 10-year follow-up of the Hoorn Study. Atherosclerosis 2007;191(2):391-396.

Szeplaki G, Prohaszka Z, Duba J, et al. Association of high serum concentration of the third component of complement (C3) with pre-existing severe coronary artery disease and new vascular events in women. Atherosclerosis 2004;177(2):383-389.

Libby P. Changing concepts of atherogenesis. J Intern Med 2000;247(3):349-358.

Eck SL, Perkins ND, Carr DP, et al. Inhibition of phorbol ester-induced cellular adhesion by competitive binding of NF-kappa B in vivo. Mol Cell Biol 1993;13(10):6530-6536.

66 Ridker PM, Hennekens CH, Buring JE, et al. C-reactive protein and other markers of inflammation in the prediction of cardiovascular disease in women. $\mathrm{N}$ Engl J Med 2000;342(12):836-843.

67 Ridker PM, Stampfer MJ, Rifai N. Novel risk factors for systemic atherosclerosis: a comparison of C-reactive protein, fibrinogen, homocysteine, lipoprotein(a), and standard cholesterol screening as predictors of peripheral arterial disease. Jama 2001;285(19):2481-2485.

68 van der Meer IM, Iglesias del Sol A, Hak AE, et al. Risk factors for progression of atherosclerosis measured at multiple sites in the arterial tree: the Rotterdam Study. Stroke 2003;34(10):2374-2379.

Aronson D, Rayfield EJ. How hyperglycemia promotes atherosclerosis: molecular mechanisms. Cardiovasc Diabetol 2002;1(1).

Cimminiello C. PAD. Epidemiology and pathophysiology. Thromb Res 2002;106(6):V295-301.

1 Corpeleijn E, van der Kallen CJ, Kruijshoop M, et al. Direct association of a promoter polymorphism in the CD36/FAT fatty acid transporter gene with Type 2 diabetes mellitus and insulin resistance. Diabet Med 2006;23(8):907-911.

Kruijshoop M, Feskens EJ, Blaak EE, et al. Validation of capillary glucose measurements to detect glucose intolerance or type 2 diabetes mellitus in the general population. Clin Chim Acta 2004;341(1-2):33-40. 
73 van Greevenbroek MM, Vermeulen VM, Feskens EJ, et al. Genetic variation in thioredoxin interacting protein (TXNIP) is associated with hypertriglyceridaemia and blood pressure in diabetes mellitus. Diabet Med 2007;24(5):498-504.

74 WHO. Definition, diagnosis and classification of diabetes mellitus. Report of a WHO Consultation. Part 1: Diagnosis and Classification of Diabetes Mellitus. Geneva, Switzerland: $\mathrm{WHO} / \mathrm{NCD} / \mathrm{NCS} / 9921999$. 



\section{Chapter 2}

Low-grade inflammation can partly explain the association between the metabolic syndrome and either coronary artery disease or severity of peripheral arterial disease: The CODAM study

Marjon Jacobs, Marleen M van Greevenbroek, Carla J van der Kallen, Isabel Ferreira, Ellen E Blaak, Edith J Feskens, Eugene H Jansen, Casper G Schalkwijk, Coen D Stehouwer 


\begin{abstract}
Background: Low-grade inflammation has been hypothesized to underlie the coronary artery disease (CAD) risk associated with the metabolic syndrome, but the evidence is not conclusive. For peripheral arterial disease (PAD; as measured by the ankle-arm index), this association has not been studied before. The aim was to study whether the association between the metabolic syndrome and CAD or the severity of PAD can be explained by low-grade inflammation. Methods: The CODAM (Cohort study Diabetes and Atherosclerosis Maastricht) population includes 574 subjects, with an increased risk of type 2 diabetes, of whom 560 were included in the analyses ( 343 males; age: $59.5 \pm 7.0$ years). The inflammation markers that were measured were C-reactive protein, interleukin 6, soluble vascular cell adhesion molecule-1, soluble intercellular adhesion molecule- 1 and serum amyloid A. All analyses were adjusted for age, sex and smoking. Results: Logistic regression showed that the metabolic syndrome was significantly associated with CAD (odds ratio $[\mathrm{OR}]=1.86,95 \% \mathrm{CI}: 1.21 ; 2.84, \mathrm{p}=0.004$ ). Further adjustment for inflammatory status, as captured in a combination of the inflammation markers (using an averaged Z-score), resulted in significant associations of both the metabolic syndrome and inflammatory status with $\mathrm{CAD}\left(\mathrm{OR}_{\text {metabolic syndrome }}(95 \% \mathrm{CI})=1.58(1.01 ; 2.46)\right.$, $\left.\mathrm{p}=0.044 ; \mathrm{OR}_{\text {inflammation }}(95 \% \mathrm{CI})=1.59(1.14 ; 2.21), \mathrm{p}=0.007\right)$. Linear regression analysis showed similar results for the ankle-arm index. Conclusions: The association between the metabolic syndrome on the one hand and prevalence of CAD or the severity of PAD on the other can be partly but not completely, 26\% and 29\% respectively, explained by low-grade inflammation.
\end{abstract}




\subsection{Introduction}

The metabolic syndrome affects at least one quarter of the population in developed countries and is associated with an increased risk of developing type 2 diabetes and cardiovascular disease [1-3]. The main components of the metabolic syndrome are abdominal obesity, hypertension, dyslipidemia and a disturbed glucose metabolism [4]. The underlying pathogenesis of the relation between the metabolic syndrome and coronary artery disease is unknown but it has been hypothesized that low-grade inflammation might be involved [5-12]. The relation between the metabolic syndrome and peripheral arterial disease has not been extensively addressed [13-16], but it is reasonable to hypothesize that similar mechanisms may be operative.

Low-grade inflammation in particular has been hypothesized to not underlie only the pathogenesis of the metabolic syndrome but also its association with cardiovascular diseases $[5,6,8,10-12]$. One important determinant of low-grade inflammation in the metabolic syndrome might be central obesity, because visceral adipose tissue may produce inflammatory mediators, which in turn induce the production of acute phase reactants in hepatocytes and endothelial cells. Indeed, the metabolic syndrome has been associated with markers of inflammatory activity such as C-reactive protein (CRP) [9, 17-25], interleukin 6 (IL6) [26, 27], serum amyloid A (SAA) [28, 29] and soluble adhesion molecules [7, 23, 25, 30].

In view of these considerations, we tested the hypothesis that the association between the metabolic syndrome on the one hand and coronary artery disease (CAD) or severity of peripheral arterial disease (PAD, as measured by the ankle-arm index [AAIx]) on the other can be explained by an increased inflammatory activity. We investigated this, cross-sectionally, in the Cohort study on Diabetes and Atherosclerosis Maastricht (CODAM).

\subsection{Materials and methods}

\subsubsection{Subjects}

The CODAM cohort [31] consists of 574 subjects who were invited from a large population based cohort on the basis of an elevated risk for type 2 diabetes (T2DM) [32]. Inclusion criteria for our cohort were Caucasian ethnicity and age older than 40 years and one or more of the following: body mass index (BMI) above $25 \mathrm{~kg} / \mathrm{m}^{2}$, a positive family history for T2DM, a history of gestational diabetes, the use of antihypertensive medication, a postprandial glucose above $6.0 \mathrm{mmol} / \mathrm{L}$ and/or glucosuria. After a first oral glucose tolerance test [33], 301 subjects had normal glucose tolerance, 127 had impaired glucose metabolism (IGM) and 146 had T2DM. IGM was defined as subjects who had either impaired glucose tolerance or impaired fasting glucose. 
$55 \%$ of the T2DM subjects were not aware of their diabetes status prior to the screening. The study was approved by the local Medical Ethical Committee of Maastricht University. All subjects gave written informed consent.

For the analyses 560 subjects were included. Eight subjects were excluded because of missing data on inflammation, 1 had missing data on the AAIx and another 2 had missing data on smoking. Three subjects had an AAIx $\geq 1.5$ and were excluded because of probable arterial rigidity.

\subsubsection{Measurements}

Waist circumference was measured at the level midway between the lateral lower rib margin and the spina iliaca anterior superior. Blood pressure was measured twice on each visit after 5 minutes of rest with an oscillometric precision blood pressure instrument (Maxi stable 3, Speidel \& Keller, currently: Welch Allyn Inc, Skaneateles Falls, New York, USA) on the right arm in the supine position. Furthermore systolic blood pressures were measured with a standard Doppler device (Mini Dopplex D900, Huntleigh Diagnostics Ltd, currently: E-Medical, Harmelen, the Netherlands) in the brachial arteries and twice in both the tibialis posterior and dorsalis pedis arteries of the lower extremities. The ankle-arm index was calculated for each leg by dividing the highest ankle pressure by the highest brachial pressure. The lowest AAIx of either leg was used in the analyses.

Lipid lowering medication was stopped 14 days before the first visit and all other medication was stopped the day before the visit. Blood samples were obtained by venipuncture to determine glucose, cholesterol, triglycerides and inflammation markers (CRP, IL6, SAA, soluble vascular cell adhesion molecule-1 (sVCAM-1), and soluble intercellular adhesion molecule-1 (sICAM-1)). Glucose was measured in NaF$\mathrm{KO}$ (sodium fluoride - potassium oxalate) plasma by the hexokinase method [31, 34]. High-density lipoprotein cholesterol (HDL-cholesterol) and triglycerides were measured in ethylenediaminetetraacetic acid (EDTA) plasma by enzymatic methods (Roche, Mannheim, Germany) by the Department of Clinical Chemistry of the University Hospital Vrije Universiteit Amsterdam (The Netherlands). CRP was measured in serum with a high-sensitivity kit (Latex, Roche Diagnostics Nederland BV, Almere, The Netherlands). IL6 and sVCAM-1 were both measured in EDTA plasma with a high-sensitivity human Quantikine Enzyme-Linked Immuno Sorbent Assay (ELISA) kit (R\&D systems, Minneapolis, Minnesota, USA). sICAM-1 and SAA were measured in EDTA plasma by ELISA (Biosource, Invitrogen, Carlsbad, California, USA). All inflammation markers were measured at the Laboratory of Toxicology, Genetics and Pathology of the National Public Health Institute, Bilthoven (The Netherlands).

The metabolic syndrome was defined according to the American Heart Association and the National Heart, Lung and Blood Institute [35]. 
Coronary artery disease was defined as self-reported myocardial infarction, bypass surgery of the coronary arteries, balloon dilatation or stent placement (through questionnaires) and/or presence of signs of myocardial infarction (Minnesota codes 11 or 1-2) or ischemia (Minnesota codes $1-3,4-1,4-2,4-3,5-1,5-2,5-3$ or 7-1) on a 12lead ECG. For the major part of the cases ( $>75 \%$ of the subjects with self-reported coronary heart disease), the self-reports could be confirmed using available hospital registries.

In the CODAM study, only 28 out of 574 subjects had AAIx $<0.9$ (the traditional criterion for the diagnosis of PAD) and thus we lacked power to address the question on PAD as a categorical variable. Therefore we used AAIx as continuous variable for the severity of PAD. This is reasonable since formation of atherosclerosis is a continuous process and every decrease of the AAIx below 1.5 is a reflection of atherosclerosis $[36,37]$.

\subsubsection{Statistical analysis}

Variables having a skewed distribution were log-transformed prior to further analyses. Firstly, logistic and linear regression analyses were used to analyse the relation between the metabolic syndrome on the one hand and CAD or the AAIx, respectively, on the other (model 1, Table 4). All analyses were adjusted for age, sex and smoking (packyears). Next, the association between the metabolic syndrome and markers of inflammation was investigated. Finally, the extent to which the association between the metabolic syndrome and CAD or the AAIx could be explained by inflammation was determined (in which case the regression coefficient of the metabolic syndrome was expected to be attenuated). P-values smaller than 0.05 were considered significant. Because the inflammation markers were measured only once, the associations between each inflammation markers with the study outcomes will tend to be underestimated. Therefore, a more robust measure reflective of subjects' levels of low-grade inflammation was calculated on the basis of the values of the several markers of inflammation investigated. Specifically, we first standardized each inflammation marker (i.e. CRP, IL6, sVCAM-1, sICAM-1 and SAA) to comparable units, by converting them into z-scores [i.e. (the subjects' observed value - mean value in the cohort)/cohort standard deviation], and then averaged these $z$-scores into a total inflammation score [38].

All statistical analyses were performed using the SPSS package version 13.0 (SPSS, Chicago, IL, USA).

\subsection{Results}

Table 2.1 shows the basic characteristics of the subjects according to the absence and presence of the metabolic syndrome. In total 560 subjects were included (343 males; 
age: $59.5 \pm 7.0$ years), of whom 54\% had the metabolic syndrome. There were significantly more subjects with impaired glucose metabolism in the group subjects with the metabolic syndrome vs. without $(27.9 \%$ vs. $15.3 \%, \mathrm{p}<0.001)$ and also more diabetics $(39.7 \%$ vs. $7.1 \%, \mathrm{p}<0.001)$. Also the prevalence of CAD was significantly higher in the group of subjects with the metabolic syndrome $(28.9 \%$ vs. $16.9 \%$, $\mathrm{p}=0.001)$ and they had a lower AAIx (1.07 vs. 1.11, $\mathrm{p}=0.001)$. 
Table 2.1: Characteristics of the CODAM cohort according to the absence or presence of the metabolic syndrome (MetS)

without MetS with MetS p-value

$$
(\mathrm{n}=255) \quad(\mathrm{n}=305)
$$

\begin{tabular}{|c|c|c|c|}
\hline Male / Female & $149 / 106$ & 194/111 & 0.211 \\
\hline Age (years) & $58.8 \pm 7.4$ & $60.2 \pm 6.6$ & 0.026 \\
\hline Smoking (pack-years) ${ }^{*}$ & $9.5(0.0-26.4)$ & $16.0(0.0-34.0)$ & 0.088 \\
\hline Waist $(\mathrm{cm})$ & $92.1 \pm 11.0$ & $104.6 \pm 10.6$ & $<0.001$ \\
\hline HDL cholesterol (mmol/L) & $1.39 \pm 0.34$ & $1.02 \pm 0.25$ & $<0.001$ \\
\hline Triglycerides $(\mathrm{mmol} / \mathrm{L})^{*}$ & $1.0(0.8-1.4)$ & $1.8(1.4-2.2)$ & $<0.001$ \\
\hline Systolic blood pressure (mmHg) & $134 \pm 19$ & $145 \pm 17$ & $<0.001$ \\
\hline Diastolic blood pressure $(\mathrm{mmHg})$ & $79 \pm 9$ & $84 \pm 9$ & $<0.001$ \\
\hline Fasting plasma glucose $(\mathrm{mmol} / \mathrm{L})$ & $5.4 \pm 0.77$ & $6.6 \pm 1.71$ & $<0.001$ \\
\hline Use of antihypertensive medication (\%) & 23.9 & 47.5 & $<0.001$ \\
\hline Use of lipid-lowering medication ( $\%)$ & 11.8 & 24.3 & $<0.001$ \\
\hline Impaired glucose metabolism (\%) & 15.3 & 27.9 & $<0.001$ \\
\hline Type 2 Diabetes (\%) & 7.1 & 39.7 & $<0.001$ \\
\hline Coronary Artery Disease (\%) & 16.9 & 28.9 & 0.001 \\
\hline Ankle-arm index & $1.11 \pm 0.11$ & $1.07 \pm 0.13$ & 0.001 \\
\hline C-reactive protein $(\mu \mathrm{g} / \mathrm{mL})^{*}$ & $2.0(1.1-3.5)$ & $3.1(1.8-5.4)$ & $<0.001$ \\
\hline Interleukin $6(\mathrm{pg} / \mathrm{mL})^{*}$ & $1.2(0.79-1.8)$ & $1.6(1.1-2.4)$ & $<0.001$ \\
\hline Soluble vascular cell adhesion molecule $1(\mathrm{ng} / \mathrm{mL})^{*}$ & $441(376-512)$ & $483(396-576)$ & $<0.001$ \\
\hline Soluble intercellular adhesion molecule $1(\mathrm{ng} / \mathrm{mL})^{*}$ & $311(272-364)$ & $354(306-419)$ & $<0.001$ \\
\hline Serum amyloid $\mathrm{A}(\mu \mathrm{g} / \mathrm{mL})^{*}$ & $6.0(3.7-13.5)$ & $7.9(4.6-14.5)$ & 0.013 \\
\hline
\end{tabular}

Data presented as number, mean $\pm \mathrm{SD}$, median (interquartile range) or percentage;

Probabilities for normal distributed continuous variables are from student t-tests and for categorical variables from Chi-square tests;

*: data were log-transformed prior to analyses 
The metabolic syndrome was a significant determinant of CAD (odds ratio (95\% confidence interval $)=1.86(1.21 ; 2.84) ; \mathrm{p}=0.004$, after adjustment for age, sex and smoking) and also of the AAIx (linear regression coefficient $\beta$ (95\% confidence interval $=-0.035(-0.055 ;-0.016) ; \mathrm{p}<0.001$, after adjustment for age, sex and smoking).

We subsequently determined whether the metabolic syndrome was associated with the individual inflammation markers and with the inflammation score (Table 2.2). Analyses showed that the metabolic syndrome was associated with all five individual inflammation markers and also with the inflammation score. Furthermore we determined whether inflammation was associated with CAD or AAIx after adjustment for age, sex, smoking and the metabolic syndrome (Table 2.3). Analyses showed that IL6 and the inflammation score were associated with CAD whereas CRP, IL6 and the inflammation score were significantly, inversely associated with the AAIx.

Table 2.2: Association between the metabolic syndrome and markers of inflammation

\begin{tabular}{lccc}
\hline Markers of inflammation & $\beta$ & $95 \% \mathrm{CI}$ & p-value \\
\hline CRP & 0.506 & $0.344-0.668$ & $<0.001$ \\
IL6 & 0.379 & $0.216-0.542$ & $<0.001$ \\
sVCAM-1 & 0.238 & $0.084-0.391$ & 0.002 \\
sICAM-1 & 0.549 & $0.388-0.710$ & $<0.001$ \\
SAA & 0.202 & $0.040-0.364$ & 0.014 \\
Total inflammation score & 0.375 & $0.271-0.479$ & $<0.001$ \\
\hline
\end{tabular}

$\beta$ : indicates differences in inflammatory markers (expressed in SD) between subjects with vs. without the metabolic syndrome; All analyses were adjusted for age, sex and smoking 
Table 2.3: Association between inflammation and coronary artery disease (CAD) or ankle-arm index (AAIx)

\begin{tabular}{|c|c|c|c|c|c|c|}
\hline \multirow[b]{3}{*}{ Main determinant } & \multicolumn{6}{|c|}{ Main outcomes } \\
\hline & \multicolumn{3}{|c|}{ CAD } & \multicolumn{3}{|c|}{ AAIx } \\
\hline & OR & $95 \% \mathrm{CI}$ & p-value & $\beta$ & $95 \% \mathrm{CI}$ & $\mathrm{p}$-value \\
\hline CRP & 1.17 & $0.94-1.45$ & 0.155 & -0.017 & $-0.027 ;-0.007$ & 0.001 \\
\hline IL6 & 1.47 & $1.18-1.81$ & $<0.001$ & -0.019 & $-0.029 ;-0.009$ & $<0.001$ \\
\hline sVCAM-1 & 1.01 & $0.81-1.26$ & 0.928 & -0.002 & $-0.013 ; 0.008$ & 0.666 \\
\hline sICAM-1 & 1.22 & $0.99-1.50$ & 0.065 & -0.010 & $-0.020 ; 0.001$ & 0.066 \\
\hline SAA & 1.23 & $0.995-1.52$ & 0.056 & -0.007 & $-0.017 ; 0.003$ & 0.164 \\
\hline$Z_{\text {Inflammation }}$ & 1.59 & $1.14-2.21$ & 0.007 & -0.027 & $-0.043 ;-0.011$ & 0.001 \\
\hline
\end{tabular}

OR: indicates association of inflammatory markers or inflammation score per one SD change with CAD; $\beta$ : indicates difference in AAIx per one SD change in inflammatory markers or inflammation score; All analyses were adjusted for age, sex, smoking and the metabolic syndrome

Finally, we determined whether the association between the metabolic syndrome on the one hand and CAD or the AAIx on the other could be explained by inflammatory activity Table 2.4). When the overall score for the inflammatory status $\left(Z_{\text {inflammation }}\right)$ was included in the original logistic regression model (model 7, Table 2.4), the association of the metabolic syndrome with CAD remained significant (OR $(95 \% \mathrm{CI})$ $=1.58(1.01 ; 2.46), \mathrm{p}=0.044)$. Note that the association of the metabolic syndrome with CAD decreased by about $26 \%$ (judged on the basis of changes in the magnitude of the regression coefficients) compared to model 1. Similarly, when the inflammation score was added to the linear regression model (model 7, Table 2.4), again the metabolic syndrome remained significantly associated with AAIx ( $\beta_{\text {metabolic syndrome }}$ $(95 \% \mathrm{CI})=-0.025(-0.046 ;-0.005), \mathrm{p}=0.015)$, with a $29 \%$ reduction of the strength of the association between the metabolic syndrome and the severity of PAD. This implies that the association between the metabolic syndrome and CAD and the AAIx, respectively, can be partly but not completely explained by low-grade inflammation. 
Table 2.4: Association between the metabolic syndrome and coronary artery disease (CAD) or ankle-arm index (AAIx) and the role of inflammation

\begin{tabular}{|c|c|c|c|c|c|c|c|}
\hline \multirow[b]{3}{*}{ Model } & \multirow[b]{3}{*}{ Adjustments } & \multicolumn{6}{|c|}{ Main outcomes } \\
\hline & & \multicolumn{3}{|c|}{ CAD } & \multicolumn{3}{|c|}{ AAIx } \\
\hline & & OR & $95 \% \mathrm{CI}$ & $\mathrm{p}$-value & $\beta$ & $95 \% \mathrm{CI}$ & p-value \\
\hline 1 & age, sex, smoking & 1.86 & $1.21-2.84$ & 0.004 & -0.035 & $-0.055 ;-0.016$ & $<0.001$ \\
\hline 2 & model $1+$ CRP & 1.73 & $1.12-2.67$ & 0.014 & -0.027 & $-0.047 ;-0.007$ & 0.010 \\
\hline 3 & model 1 + IL6 & 1.65 & $1.07-2.55$ & 0.024 & -0.028 & $-0.048 ;-0.008$ & 0.005 \\
\hline 4 & model 1 + sVCAM-1 & 1.85 & $1.21-2.84$ & 0.005 & -0.035 & $-0.055 ;-0.015$ & 0.001 \\
\hline 5 & model $1+$ sICAM-1 & 1.67 & $1.07-2.59$ & 0.023 & -0.030 & $-0.051 ;-0.010$ & 0.004 \\
\hline 6 & model $1+$ SAA & 1.79 & $1.17-2.75$ & 0.008 & -0.034 & $-0.054 ;-0.014$ & 0.001 \\
\hline 7 & model $1+$ inflammation score & 1.58 & $1.01-2.46$ & 0.044 & -0.025 & $-0.046 ;-0.005$ & 0.015 \\
\hline
\end{tabular}

OR: indicates association of the metabolic syndrome with CAD; $\beta$ : indicates difference in ankle arm index (AAIx) between subjects with vs. without the metabolic syndrome

\subsubsection{Additional analyses}

The definition of the metabolic syndrome as used in the analyses already includes use of antihypertensive, lipid-lowering and blood glucose-lowering medication. Therefore they were not added as variables to the model. For the AAIx also an upper limit of 1.4 or even 1.3 is used instead of 1.5. When 1.4 was used in our cohort, 559 subjects were included in the analyses instead of 550 subjects and 541 were included if a limit of 1.3 was used. For both upper limits the results did not change (data not shown).

\subsection{Discussion}

The main finding of this study was that the association between the metabolic syndrome on the one hand and CAD or the severity of PAD (as measured by the AAIx) on the other can be partly, but not completely, explained by inflammatory activity.

An important asset of our study was the use of a robust measure of low-grade inflammation by combining inflammation markers (CRP, IL6, sVCAM-1, sICAM-1 and SAA) while most other studies reported on associations with single inflammation markers, primarily CRP $[5,6,10,12,13,39-41]$. In general, our data are consistent with previous studies with respect to the association of both the metabolic syndrome $[8,11]$ and low-grade inflammation $[5,6,8,10-12,30,42-46]$ with CAD or the metabolic syndrome [13-16] and low-grade inflammation [13, 39-41, 47, 48] with PAD. CRP has generally been reported to remain associated with CAD even when the 
metabolic syndrome was included in the analyses [5, 6, 10, 12], and a few studies have described similar results for IL6, sometimes in combination with CRP [8, 46] with similar results. An inflammation score has not been tested before.

It is noteworthy that very few of these previous studies have actually addressed the hypothesis, and the extent to which, the association between the metabolic syndrome and CAD could be explained by low-grade inflammation, in which case the addition of inflammation markers to the model would result in an attenuation of the strength of the association between the metabolic syndrome and CAD. In our analyses, the general inflammation score was independently associated with both CAD and the metabolic syndrome and explained their relationship to some extent but not completely. In addition, no study has addressed an association of the metabolic syndrome and an inflammation score with PAD before. Most studies addressed either the association of the metabolic syndrome [13-16] with AAIx or the association of low-grade inflammation [13, 39-41, 47, 48] with AAIx. Because CAD and PAD can be viewed as different manifestations of a single pathophysiological entity, i.e. atherosclerotic disease, and indeed are closely related in middle-aged and elderly populations [49], it is reasonable to test the hypothesis that associations of PAD with the metabolic syndrome and inflammation may be similar to those with CAD. Indeed, our results support this hypothesis. Our findings thus show that the metabolic syndrome is a risk factor for both CAD and PAD, and that inflammation explains some of this risk, but other mechanisms linking the metabolic syndrome to CAD and PAD are likely to be at least as important as inflammatory activity.

Some limitations of our study are its cross-sectional design, which precludes conclusions on causality. Thus it cannot be excluded that part of the inflammatory response was caused by, rather than was a cause of, CAD or PAD. Prospective studies are needed to further evaluate this. Additionally, we studied middle-aged Caucasian individuals, and we therefore do not know whether our results can be extrapolated to other ethnicities or age groups. Furthermore, we studied five markers of inflammation but cannot exclude that additional inflammatory markers, which may reflect other components of the complex inflammatory response, might to some extent explain the association between the metabolic syndrome on the one hand and CAD or the severity of PAD on the other hand.

In conclusion, both the metabolic syndrome and low-grade inflammation were associated with both CAD and the severity of PAD (as measured by the AAIx), respectively. The association between the metabolic syndrome and prevalence of CAD or the severity of PAD was explained partly but not completely by low-grade inflammation because a reduction in the regression coefficients for CAD and AAIx, respectively was found, while the metabolic syndrome did remain independently associated with the outcome. 


\subsection{References}

1 Alexander CM, Landsman PB, Teutsch SM and Haffner SM. NCEP-defined metabolic syndrome, diabetes, and prevalence of coronary heart disease among NHANES III participants age 50 years and older. Diabetes 2003;52:1210-4.

2 Fonseca VA. The metabolic syndrome, hyperlipidemia, and insulin resistance. Clin Cornerstone 2005;7:61-72.

3 Lakka HM, Laaksonen DE, Lakka TA, Niskanen LK, Kumpusalo E, Tuomilehto J et al. The metabolic syndrome and total and cardiovascular disease mortality in middle-aged men. Jama 2002;288:2709-16.

4 Third Report of the National Cholesterol Education Program (NCEP) Expert Panel on Detection, Evaluation, and Treatment of High Blood Cholesterol in Adults (Adult Treatment Panel III) final report. Circulation 2002;106:3143-421.

5 Dai DF, Lin JW, Kao JH, Hsu CN, Chiang FT, Lin JL et al. The effects of metabolic syndrome versus infectious burden on inflammation, severity of coronary atherosclerosis, and major adverse cardiovascular events. J Clin Endocrinol Metab 2007;92:2532-7.

6 Haffner SM. The metabolic syndrome: inflammation, diabetes mellitus, and cardiovascular disease. Am J Cardiol 2006;97:3A-11A.

$7 \quad$ Koh KK, Han SH and Quon MJ. Inflammatory markers and the metabolic syndrome: insights from therapeutic interventions. J Am Coll Cardiol 2005;46:1978-85.

8 Langenberg C, Bergstrom J, Scheidt-Nave C, Pfeilschifter J and Barrett-Connor E. Cardiovascular death and the metabolic syndrome: role of adiposity-signaling hormones and inflammatory markers. Diabetes Care 2006;29:1363-9.

9 Leinonen E, Hurt-Camejo E, Wiklund O, Hulten LM, Hiukka A and Taskinen MR. Insulin resistance and adiposity correlate with acute-phase reaction and soluble cell adhesion molecules in type 2 diabetes. Atherosclerosis 2003;166:387-94.

10 Malik S, Wong ND, Franklin S, Pio J, Fairchild C and Chen R. Cardiovascular disease in U.S. patients with metabolic syndrome, diabetes, and elevated C-reactive protein. Diabetes Care 2005;28:690-3.

11 Pischon T, Hu FB, Rexrode KM, Girman CJ, Manson JE and Rimm EB. Inflammation, the metabolic syndrome, and risk of coronary heart disease in women and men. Atherosclerosis 2008;197:392-9.

12 Rutter MK, Meigs JB, Sullivan LM, D'Agostino RB, Sr. and Wilson PW. C-reactive protein, the metabolic syndrome, and prediction of cardiovascular events in the Framingham Offspring Study. Circulation 2004;110:380-5.

13 Brevetti G, Schiano V, Sirico G, Giugliano G, Laurenzano E and Chiariello M. Metabolic syndrome in peripheral arterial disease: relationship with severity of peripheral circulatory insufficiency, inflammatory status, and cardiovascular comorbidity. J Vasc Surg 2006;44:101-7; discussion 7. 

syndrome is associated with advanced vascular damage in patients with coronary heart disease, stroke, peripheral arterial disease or abdominal aortic aneurysm. Eur Heart J 2004;25:342-8. Taniguchi A, Fukushima M, Kuroe A, Sakaguchi K, Hashimoto H, Yoshioka I et al. Metabolic syndrome, insulin resistance, and atherosclerosis in Japanese type 2 diabetic patients. Metabolism 2007;56:1099-103.

16 Wild SH, Byrne CD, Smith FB, Lee AJ and Fowkes FG. Low ankle-brachial pressure index predicts increased risk of cardiovascular disease independent of the metabolic syndrome and conventional cardiovascular risk factors in the Edinburgh Artery Study. Diabetes Care 2006;29:637-42.

17 Anty R, Bekri S, Luciani N, Saint-Paul MC, Dahman M, Iannelli A et al. The inflammatory Creactive protein is increased in both liver and adipose tissue in severely obese patients independently from metabolic syndrome, Type 2 diabetes, and NASH. Am J Gastroenterol 2006;101:1824-33.

18 Calabro P, Chang DW, Willerson JT and Yeh ET. Release of C-reactive protein in response to inflammatory cytokines by human adipocytes: linking obesity to vascular inflammation. J Am Coll Cardiol 2005;46:1112-3.

19 Florez H, Castillo-Florez S, Mendez A, Casanova-Romero P, Larreal-Urdaneta C, Lee D et al. C-reactive protein is elevated in obese patients with the metabolic syndrome. Diabetes Res Clin Pract 2006;71:92-100.

20 Gonzalez AS, Guerrero DB, Soto MB, Diaz SP, Martinez-Olmos M and Vidal O. Metabolic syndrome, insulin resistance and the inflammation markers C-reactive protein and ferritin. Eur J Clin Nutr 2006;60:802-9.

21 Ouchi N, Kihara S, Funahashi T, Nakamura T, Nishida M, Kumada M et al. Reciprocal association of C-reactive protein with adiponectin in blood stream and adipose tissue. Circulation 2003;107:671-4.

22 Van Guilder GP, Hoetzer GL, Greiner JJ, Stauffer BL and Desouza CA. Influence of metabolic syndrome on biomarkers of oxidative stress and inflammation in obese adults. Obesity (Silver Spring) 2006;14:2127-31.

23 Ingelsson E, Hulthe J and Lind L. Inflammatory markers in relation to insulin resistance and the metabolic syndrome. Eur J Clin Invest 2008;38:502-9.

24 Matsuo Y, Hashizume T, Shioji S and Akasaka T. Metabolic Syndrome is Strongly Associated With Chronic Subclinical Inflammation in Patients Achieving Optimal Low-Density Lipoprotein-Cholesterol Levels in Secondary Prevention of Cardiovascular Disease. Circ J 2008;72:2046-50.

25 Kressel G, Trunz B, Bub A, Hulsmann O, Wolters M, Lichtinghagen R et al. Systemic and vascular markers of inflammation in relation to metabolic syndrome and insulin resistance in adults with elevated atherosclerosis risk. Atherosclerosis 2009;202:263-71.

26 Bataille $\mathrm{R}$ and Klein B. C-reactive protein levels as a direct indicator of interleukin-6 levels in humans in vivo. Arthritis Rheum 1992;35:982-4. 
Heinrich PC, Castell JV and Andus T. Interleukin-6 and the acute phase response. Biochem J 1990;265:621-36.

Castell JV, Gomez-Lechon MJ, David M, Andus T, Geiger T, Trullenque R et al. Interleukin-6 is the major regulator of acute phase protein synthesis in adult human hepatocytes. FEBS Lett 1989;242:237-9.

Castell JV, Gomez-Lechon MJ, David M, Hirano T, Kishimoto T and Heinrich PC. Recombinant human interleukin-6 (IL-6/BSF-2/HSF) regulates the synthesis of acute phase proteins in human hepatocytes. FEBS Lett 1988;232:347-50.

Haught WH, Mansour M, Rothlein R, Kishimoto TK, Mainolfi EA, Hendricks JB et al. Alterations in circulating intercellular adhesion molecule-1 and L-selectin: further evidence for chronic inflammation in ischemic heart disease. Am Heart J 1996;132:1-8.

1 Kruijshoop M, Feskens EJ, Blaak EE and de Bruin TW. Validation of capillary glucose measurements to detect glucose intolerance or type 2 diabetes mellitus in the general population. Clin Chim Acta 2004;341:33-40.

van Dam RM, Boer JM, Feskens EJ and Seidell JC. Parental history of diabetes modifies the association between abdominal adiposity and hyperglycemia. Diabetes Care 2001;24:1454-9.

WHO. Definition, diagnosis and classification of diabetes mellitus. Report of a WHO Consultation. Part 1: Diagnosis and Classification of Diabetes Mellitus. Geneva, Switzerland: WHO/NCD/NCS/99.2. 1999.

4 Mensink M, Corpeleijn E, Feskens EJ, Kruijshoop M, Saris WH, de Bruin TW et al. Study on lifestyle-intervention and impaired glucose tolerance Maastricht (SLIM): design and screening results. Diabetes Res Clin Pract 2003;61:49-58.

5 Grundy SM, Cleeman JI, Daniels SR, Donato KA, Eckel RH, Franklin BA et al. Diagnosis and management of the metabolic syndrome: an American Heart Association/National Heart, Lung, and Blood Institute Scientific Statement. Circulation 2005;112:2735-52.

Aboyans V, Criqui MH, Denenberg JO, Knoke JD, Ridker PM and Fronek A. Risk factors for progression of peripheral arterial disease in large and small vessels. Circulation 2006;113:26239.

McDermott MM, Green D, Greenland P, Liu K, Criqui MH, Chan C et al. Relation of levels of hemostatic factors and inflammatory markers to the ankle brachial index. Am J Cardiol 2003;92:194-9.

Schram MT, Chaturvedi N, Schalkwijk C, Giorgino F, Ebeling P, Fuller JH et al. Vascular risk factors and markers of endothelial function as determinants of inflammatory markers in type 1 diabetes: the EURODIAB Prospective Complications Study. Diabetes Care 2003;26:2165-73.

Chang ST, Chen CL, Chu CM, Lin PC, Chung CM, Hsu JT et al. Ankle-arm index is a useful test for clinical practice in outpatients with suspected coronary artery disease. Circ J 2006;70:686-90.

Shankar A, Li J, Nieto FJ, Klein BE and Klein R. Association between C-reactive protein level and peripheral arterial disease among US adults without cardiovascular disease, diabetes, or hypertension. Am Heart J 2007;154:495-501. 
Vu JD, Vu JB, Pio JR, Malik S, Franklin SS, Chen RS et al. Impact of C-reactive protein on the likelihood of peripheral arterial disease in United States adults with the metabolic syndrome, diabetes mellitus, and preexisting cardiovascular disease. Am J Cardiol 2005;96:655-8. De Backer J, Mak R, De Bacquer D, Van Renterghem L, Verbraekel E, Kornitzer M et al. Parameters of inflammation and infection in a community based case-control study of coronary heart disease. Atherosclerosis 2002;160:457-63. Mojiminiyi OA, Abdella N, Moussa MA, Akanji AO, Al Mohammedi H and Zaki M. Association of $\mathrm{C}$-reactive protein with coronary heart disease risk factors in patients with type 2 diabetes mellitus. Diabetes Res Clin Pract 2002;58:37-44.

Ridker PM, Hennekens CH, Roitman-Johnson B, Stampfer MJ and Allen J. Plasma concentration of soluble intercellular adhesion molecule 1 and risks of future myocardial infarction in apparently healthy men. Lancet 1998;351:88-92.

Schumacher A, Seljeflot I, Sommervoll L, Christensen B, Otterstad JE and Arnesen H. Increased levels of markers of vascular inflammation in patients with coronary heart disease. Scand J Clin Lab Invest 2002;62:59-68. Wannamethee SG, Whincup PH, Rumley A and Lowe GD. Inter-relationships of interleukin-6, cardiovascular risk factors and the metabolic syndrome among older men. J Thromb Haemost 2007;5:1637-43.

47 Tzoulaki I, Murray GD, Lee AJ, Rumley A, Lowe GD and Fowkes FG. C-reactive protein, interleukin-6, and soluble adhesion molecules as predictors of progressive peripheral atherosclerosis in the general population: Edinburgh Artery Study. Circulation 2005;112:976-83. van der Meer IM, de Maat MP, Bots ML, Breteler MM, Meijer J, Kiliaan AJ et al. Inflammatory mediators and cell adhesion molecules as indicators of severity of atherosclerosis: the Rotterdam Study. Arterioscler Thromb Vasc Biol 2002;22:838-42. prevalence of peripheral arterial disease in a defined population. Circulation 1985;71:510-5 



\section{Chapter 3}

\section{The association between the metabolic syndrome and peripheral, but not coronary, artery disease is partly mediated by endothelial dysfunction: The CODAM study}




\begin{abstract}
Objective: The metabolic syndrome is associated with coronary artery disease (CAD) and with peripheral artery disease (PAD) but the underlying mechanisms explaining these associations have not yet been completely clarified. The aim was to investigate: 1) whether endothelial dysfunction can explain the association between the metabolic syndrome and CAD and/or the severity of PAD, as measured by the ankle-arm index (AAIx); and 2) whether any such mediation is independent of that from low-grade inflammation. Methods: We studied 539 subjects $(232$ men) aged $59.4 \pm 6.9$ years, with an increased risk of type 2 diabetes and cardiovascular diseases. Endothelial dysfunction and inflammation scores were calculated from 3 markers of endothelial dysfunction (soluble E-selectin, soluble vascular cell adhesion molecule- 1 and von Willebrand factor) and 6 of inflammation (C-reactive protein, interleukin 6, soluble intercellular adhesion molecule-1, serum amyloid A, ceruloplasmin, haptoglobin). The association between the metabolic syndrome and CAD and/or PAD, and the mediating role of endothelial dysfunction herein was examined with logistic and linear regression analyses, all adjusted for age, sex and smoking. Results: Subjects with the metabolic syndrome $(\mathrm{n}=289 ; 54 \%)$ had higher prevalence of $\mathrm{CAD}(\mathrm{OR}(95 \% \mathrm{CI})=1.75(1.14 ; 2.69))$ and lower AAIx $(\beta(95 \% \mathrm{CI})=-0.036(-0.056 ;-0.016))$. Endothelial dysfunction explained $6 \%$ of the association between the metabolic syndrome and CAD, and $19 \%$ of the association with AAIx, whereas low-grade inflammation explained $26 \%$ and $28 \%$ of these associations, respectively. Together, the two scores explained $24 \%$ and $36 \%$ of the association between the metabolic syndrome and CAD and AAIx, respectively. Conclusion: Endothelial dysfunction explains part of association between the metabolic syndrome and the severity of PAD but is not involved in the association between the metabolic syndrome and CAD. This indicates that the pathophysiologies of coronary and peripheral artery disease are essentially distinct.
\end{abstract}




\subsection{Introduction}

The metabolic syndrome affects at least one quarter of the population of developed countries and its prevalence is rapidly increasing. The metabolic syndrome is associated with an increased risk of type 2 diabetes mellitus (T2DM) as well as cardiovascular diseases (CVD) [1, 2], but the underlying mechanisms explaining these associations have not yet been completely clarified.

The metabolic syndrome is associated with endothelial dysfunction and low-grade inflammation [3-5], which are key features in the pathophysiology of atherosclerosis and atherotrombosis [6-8]. Therefore, it is plausible to hypothesize that endothelial dysfunction and low-grade inflammation may explain, at least in part, the association between the metabolic syndrome and CVD. Indeed, several studies have supported the concept that low-grade inflammation mediates a part of the association between the metabolic syndrome and coronary artery disease (CAD) and peripheral arterial disease (PAD) [9-11]. Using an inflammation score that comprised 5 markers of inflammation, we recently showed that approximately $28 \%$ of the association between the metabolic syndrome and CAD or the severity of PAD was explained by low-grade inflammation [12]. However a great deal of these associations remained unexplained thus suggesting that other mechanisms must play a role as well. Endothelial dysfunction, which is associated with increased cardiovascular risk [13, 14] and is elevated in the metabolic syndrome [3,5], is a good candidate in this respect. However, endothelial dysfunction and low-grade inflammation are closely related [15] and it is therefore not clear to what extent the two mechanisms represent distinct or overlapping pathways linking the metabolic syndrome to increased risk of CAD or PAD severity.

In view of these considerations, we have therefore investigated first: the extent to which the association between the metabolic syndrome and CAD or the severity of PAD could be explained by endothelial dysfunction; and second: whether any such mediating role of endothelial dysfunction added to, or overlapped with, that already shown for low-grade inflammation. We addressed these questions, cross-sectionally, in the Cohort study on Diabetes and Atherosclerosis Maastricht (CODAM).

\subsection{Materials and methods}

\subsubsection{Subjects and study design}

The CODAM study is an ongoing prospective cohort study in the Netherlands that was originally designed to study the effects of obesity, glucose tolerance, lipid metabolism, lifestyle and genetics on cardiovascular complications, and that has been described in detail elsewhere [16]. Briefly, between 1999 and 2001 subjects were 
selected from a large population-based cohort on the basis of an elevated risk for type 2 diabetes (T2DM) [17]. Inclusion criteria for our cohort were Caucasian ethnicity, age older than 40 years and one or more of the following: body mass index above 25 $\mathrm{kg} / \mathrm{m}^{2}$, a positive family history for T2DM, a history of gestational diabetes, use of anti-hypertensive medication, a postprandial glucose above $6.0 \mathrm{mmol} / \mathrm{L}$ and/or glucosuria. In total 574 subjects were included and were extensively characterized with regard to their metabolic, cardiovascular and lifestyle risk profiles during two visits to the University's research unit [16]. The study was approved by the Medical Ethical Committee of the Maastricht University and all subjects gave written informed consent.

The present study reports on cross-sectional analyses concerning 539 subjects (232 men); those who had missing data on one or more of the markers of low-grade inflammation or endothelial dysfunction considered $(n=30)$, in whom lifetime smoking exposure could not be ascertained $(n=2)$, and who had an ankle-arm index $(A A I x) \geq 1.5$, indicating probable arterial calcification $(n=3)$ were excluded from the analyses.

\subsubsection{Metabolic syndrome}

The metabolic syndrome was defined according to the updated definition of the American Heart Association and the National Heart, Lung and Blood Institute 2005 [18], as shown in Table 3.1. Waist circumference was measured at the level midway between the lateral lower rib margin and the spina iliaca anterior superior [19]. Blood pressure was measured twice on each visit after 5 minutes of rest with an oscillometric precision blood pressure instrument (Maxi stable 3, Speidel \& Keller, currently: Welch Allyn Inc, Skaneateles Falls, New York, USA) on the right arm in the supine position. Smoking habits throughout life as well as the current use of medication was assessed by questionnaires. Subjects were asked to stop their lipid-lowering medication 14 days before the first visit and all other medication the day before the visit. Blood samples were obtained by venipuncture to determine glucose, cholesterol, and triglycerides as described in detail [12]. 
Table 3.1: Cut-off values of the components of the metabolic syndrome [18]

\begin{tabular}{ll}
\hline Component* & Categorical cut off value \\
\hline Elevated waist circumference & $\geq 102 \mathrm{~cm}$ in men \\
& $\geq 88 \mathrm{~cm}$ in women \\
& $\geq 1.7 \mathrm{mmol} / \mathrm{L}$, or on drug treatment for elevated triglycerides \\
Elevated triglycerides & $\leq 1.03 \mathrm{mmol} / \mathrm{L}$ in men \\
Reduced HDL-cholesterol & $\leq 1.3 \mathrm{mmol} / \mathrm{L}$ in women, or on drug treatment for reduced HDL-cholesterol \\
& $\geq 130 \mathrm{mmHg}$ systolic blood pressure \\
Elevated blood pressure & $\geq 85 \mathrm{mmHg}$ diastolic blood pressure, or on drug treatment for elevated blood \\
& pressure \\
& $\geq 100 \mathrm{mg} / \mathrm{dL}$ (5.6 mmol/L), or on drug treatment for elevated fasting blood \\
Elevated fasting glucose & glucose
\end{tabular}

* any 3 out of 5 constitute diagnosis of the metabolic syndrome

\subsubsection{Coronary artery disease and peripheral arterial disease}

CAD was defined as self-reported myocardial infarction, bypass surgery of the coronary arteries, balloon dilatation or stent placement (through questionnaires) and/or the presence of signs of myocardial infarction (Minnesota codes 1-1 or 1-2) or ischemia (Minnesota codes 1-3, 4-1, 4-2, 4-3, 5-1, 5-2, 5-3 or 7-1) on a 12-lead electrocardiogram [12].

PAD was defined as an AAIx $<0.9$ or a non-traumatic amputation. Because only 28 out of 539 subjects had PAD we lacked power to address the association between the metabolic syndrome and PAD as a dichotomous variable. Therefore we used AAIx as a continuous variable as a marker of the severity of PAD. This is reasonable since atherosclerosis is a continuous process and every decrease of the AAIx below 1.5 is a reflection of atherosclerosis $[20,21]$. Systolic blood pressures were measured with a standard Doppler device (Mini Dopplex D900, Huntleigh Diagnostics Ltd, currently: E-Medical, Harmelen, the Netherlands) in the brachial arteries and twice in both the tibialis posterior and dorsalis pedis arteries of the lower extremities. The AAIx was calculated for each leg by dividing the highest ankle pressure by the highest brachial pressure [22]. The lowest AAIx of either leg was used in the analyses.

\subsubsection{Markers on low-grade inflammation and endothelial dysfunction}

We measured six markers of low-grade inflammation, i.e. C-reactive protein (CRP), interleukin 6 (IL6), serum amyloid A (SAA), soluble intercellular adhesion molecule-1 (sICAM-1), ceruloplasmin and haptoglobin. These markers have been implicated in 
various aspects of the general inflammatory response [23, 24]. In addition, we measured 3 markers of endothelial dysfunction, i.e. soluble E-selectin (sE-selectin), soluble vascular cell adhesion molecule-1 (sVCAM-1), and von Willebrand factor (vWF). These circulating biomarkers have been associated with endothelial dysfunction in various studies [25-27]. Details describing the measurement of CRP, IL6, SAA, sICAM-1 and sVCAM-1 have been published before [12]. Haptoglobin and ceruloplasmin were measured in serum using Tina-quant haptoglobin and immunoturbidimetric assays, respectively (Roche Diagnostics Netherlands BV, Almere the Netherlands) on an automatic analyser. Soluble E-selectin was measured in EDTA plasma with an Elipair ELISA (Diaclone, Tepnel, Besancon Cedex, France). vWF was measured in citrate plasma as previously described [28].

\subsubsection{Statistical analysis}

Variables with a skewed distribution, i.e. triglycerides, CRP, IL6, sICAM-1, SAA, sEselectin, sVCAM-1 and vWF were log-transformed prior to further analyses. Comparisons of characteristics between subjects with vs. those without the metabolic syndrome were performed by means of Student's t-tests for continuous variables and by Chi-square tests for categorical variables.

We computed a general inflammation score by averaging the z-scores [i.e. (the subjects' observed value - the mean value in the cohort) / standard deviation of the cohorts' mean] of the six markers of low-grade inflammation considered (i.e. CRP, IL6, SAA, sICAM-1, ceruloplasmin and haptoglobin) [29]. Likewise, an endothelial dysfunction score was calculated by averaging the z-scores of sE-selectin, sVCAM-1 and vWF. These composite measures of inflammation and endothelial dysfunction were calculated to overcome the problems of multiple testing and misclassification, the later often leading to an underestimation of the strength of the associations that could occur when analysing each marker separately. Moreover, not one biomarker can be appointed that solely represents either endothelial dysfunction or a general inflammatory response. Our current approach aims to minimize that particular limitation of the use of biomarkers as surrogates of biological processes.

Linear regression analyses were used to determine first, the association of the metabolic syndrome with CAD and AAIx and also the association of the metabolic syndrome with endothelial dysfunction and with low-grade inflammation. Next, we determined whether endothelial dysfunction and low-grade inflammation were associated with either CAD or AAIx, using logistic and linear regression analyses, respectively. Finally, we examined the extent to which the associations between the metabolic syndrome and CAD or the AAIx were explained by endothelial dysfunction (in which case the magnitude of the logistic or linear regression coefficient reflecting the association between the metabolic syndrome and study outcome was expected to be attenuated after adjustment for endothelial dysfunction), and the extent to which 
any such mediation was additional to that from low-grade inflammation (by further adjusting the associations mentioned above for low-grade inflammation). All analyses were adjusted for age, sex and lifetime smoking (in pack-years). Since the definition of the metabolic syndrome already includes the use of anti-hypertensive, lipid-lowering and blood glucose-lowering medication, these were not added to the model as potential confounding variables.

All statistical analyses were performed using the SPSS package version 15.0 (SPSS, Chicago, IL, USA) and statistically significance was set at $\mathrm{p}<0.05$.

\subsection{Results}

Table 3.2 shows the general characteristics of the study population according to the presence $(n=289,54 \%$ ) of the metabolic syndrome. As compared with individuals without, those with the metabolic syndrome more often had CAD $(29.1 \%$ vs. $17.2 \%)$ and had significantly lower AAIx $(1.08 \pm 0.13$ vs. $1.11 \pm 0.11)$. Levels of all markers of low-grade inflammation and endothelial dysfunction were significantly higher in the subjects with the metabolic syndrome, except for ceruloplasmin. 
Table 3.2: Characteristics of the CODAM study population according to the absence or presence of the metabolic syndrome (MetS)

\begin{tabular}{|c|c|c|c|}
\hline & $\begin{array}{l}\text { without MetS } \\
\qquad(\mathrm{n}=250)\end{array}$ & $\begin{array}{l}\text { with MetS } \\
(\mathrm{n}=289)\end{array}$ & p-value \\
\hline Men / Women (n) & $146 / 104$ & $186 / 103$ & 0.156 \\
\hline Age (years) & $58.8 \pm 7.3$ & $60.2 \pm 6.6$ & 0.022 \\
\hline \multicolumn{4}{|l|}{ Smoking status } \\
\hline Never / ex- / current smokers (\%) & $35.2 / 46.4 / 18.4$ & $23.5 / 55.0 / 21.4$ & 0.012 \\
\hline Lifetime smoking (pack-years) $^{*}$ & $20.3(10.0-35.7)$ & $25.0(12.6-39.8)$ & 0.052 \\
\hline Waist (cm) & $92.8 \pm 9.9$ & $104.7 \pm 10.7$ & $<0.001$ \\
\hline Body Mass Index & $26.5 \pm 3.4$ & $30.2 \pm 4.2$ & $<0.001$ \\
\hline HDL cholesterol (mmol/L) & $1.40 \pm 0.34$ & $1.02 \pm 0.25$ & $<0.001$ \\
\hline Triglycerides (mmol/L) & $1.0(0.8-1.4)$ & $1.8(1.4-2.2)$ & $<0.001$ \\
\hline Systolic blood pressure $(\mathrm{mmHg})$ & $134.3 \pm 19.2$ & $144.7 \pm 16.8$ & $<0.001$ \\
\hline Diastolic blood pressure (mmHg) & $79.0 \pm 8.6$ & $84.0 \pm 8.9$ & $<0.001$ \\
\hline Fasting plasma glucose $(\mathrm{mmol} / \mathrm{L})$ & $5.4 \pm 0.8$ & $6.6 \pm 1.7$ & $<0.001$ \\
\hline Use of antihypertensive medication (\%) & 23.6 & 48.4 & $<0.001$ \\
\hline Use of lipid-lowering medication (\%) & 12.0 & 25.3 & $<0.001$ \\
\hline \multicolumn{4}{|l|}{ Glucose metabolism status } \\
\hline Normal / impaired / type 2 diabetes (\%) & $77.2 / 15.6 / 7.2$ & $31.5 / 29.1 / 39.4$ & $<0.001$ \\
\hline Coronary Artery Disease (\%) & 17.2 & 29.1 & 0.001 \\
\hline Ankle-arm index & $1.11 \pm 0.11$ & $1.08 \pm 0.13$ & 0.001 \\
\hline C-reactive protein $(\mu \mathrm{g} / \mathrm{mL})$ & $2.0(1.1-3.5)$ & $3.1(1.8-5.4)$ & $<0.001$ \\
\hline Interleukin $6(\mathrm{pg} / \mathrm{mL})$ & $1.14(0.79-1.79)$ & $1.66(1.10-2.35)$ & $<0.001$ \\
\hline Soluble intercellular adhesion molecule-1 (ng/mL) & $310(272-363)$ & $354(306-413)$ & $<0.001$ \\
\hline Serum amyloid $\mathrm{A}(\mu \mathrm{g} / \mathrm{mL})$ & $6.1(3.7-13.4)$ & $7.8(4.5-14.5)$ & 0.018 \\
\hline Ceruloplasmin $(\mathrm{g} / \mathrm{L})$ & $0.27 \pm 0.05$ & $0.26 \pm 0.06$ & 0.303 \\
\hline Haptoglobin $(\mathrm{g} / \mathrm{L})$ & $1.17 \pm 0.48$ & $1.43 \pm 0.51$ & $<0.001$ \\
\hline Inflammation score & $-0.19 \pm 0.63$ & $0.16 \pm 0.67$ & $<0.001$ \\
\hline Soluble E-selectin (ng/mL) & $66(47-88)$ & $94(70-123)$ & $<0.001$ \\
\hline Soluble vascular cell adhesion molecule-1 $(\mathrm{ng} / \mathrm{mL})$ & $441(377-511)$ & $479(393-576)$ & 0.001 \\
\hline Von Willebrand factor $(\%)$ & $110(86-154)$ & $124(92-167)$ & 0.038 \\
\hline Endothelial dysfunction score & $-0.22 \pm 0.56$ & $0.19 \pm 0.63$ & $<0.001$ \\
\hline
\end{tabular}

Data presented as number, mean $\pm \mathrm{SD}$, median (inter-quartile range) or percentage;

* estimated among ever-smokers. 
The metabolic syndrome was associated with CAD (odds ratio [OR] $(95 \% \mathrm{CI})=1.79$ $(1.17 ; 2.76), \mathrm{p}=0.008)$ and with AAIx (linear regression coefficient $\beta(95 \% \mathrm{CI})=$ -0.036 (-0.056; -0.016), $\mathrm{p}=0.001)$ (Table 3.4, model 2). The metabolic syndrome was associated with endothelial dysfunction as well as with low-grade inflammation, after adjustment for age, sex and smoking $(\beta(95 \% \mathrm{CI})=0.365(0.266 ; 0.464)$, and $\beta(95 \%$ $\mathrm{CI})=0.352(0.245 ; 0.459)$, respectively; both $\mathrm{p}<0.001)$.

Inflammation but not endothelial dysfunction was significantly associated with CAD while both endothelial dysfunction and inflammation were significantly inversely associated with the AAIx. These associations were still significant after adjustments for other risk factors and the metabolic syndrome (Table 3.3).

The magnitude of the associations between the metabolic syndrome and CAD and the AAIx were only slightly $(6 \%)$ and modestly $(19 \%)$ attenuated, respectively, when adjusted for endothelial dysfunction (Table 3.4, model 3 vs. model 2), but were attenuated by $24 \%$ and $28 \%$, respectively, when adjusted for low-grade inflammation instead (Table 3.4, model 4 vs. model 2). Together, endothelial dysfunction and lowgrade inflammation explained $23 \%$ and $36 \%$ of the association between the metabolic syndrome and CAD and AAIx, respectively (Table 3.4, model 5 vs. model 2). After considering low-grade inflammation, endothelial dysfunction thus explained an additional $8 \%$ but none of the strength of the association between the metabolic syndrome and the AAIx and CAD, respectively. 


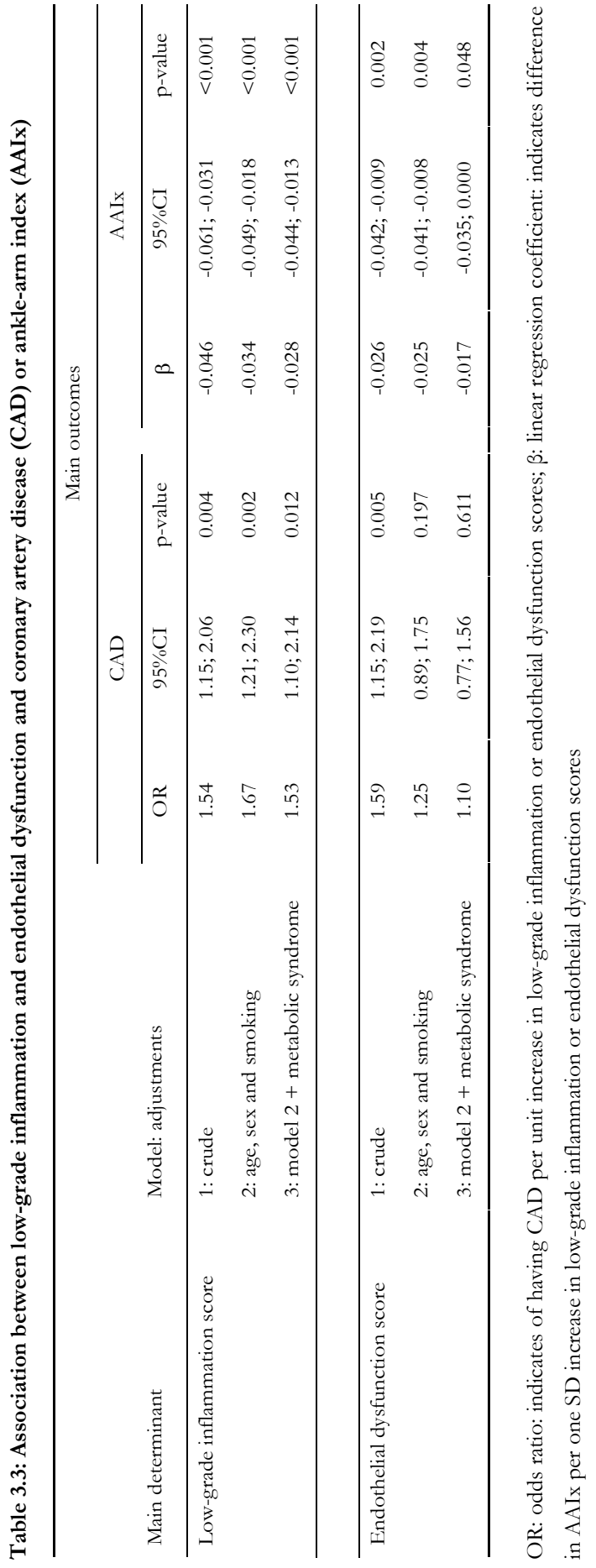




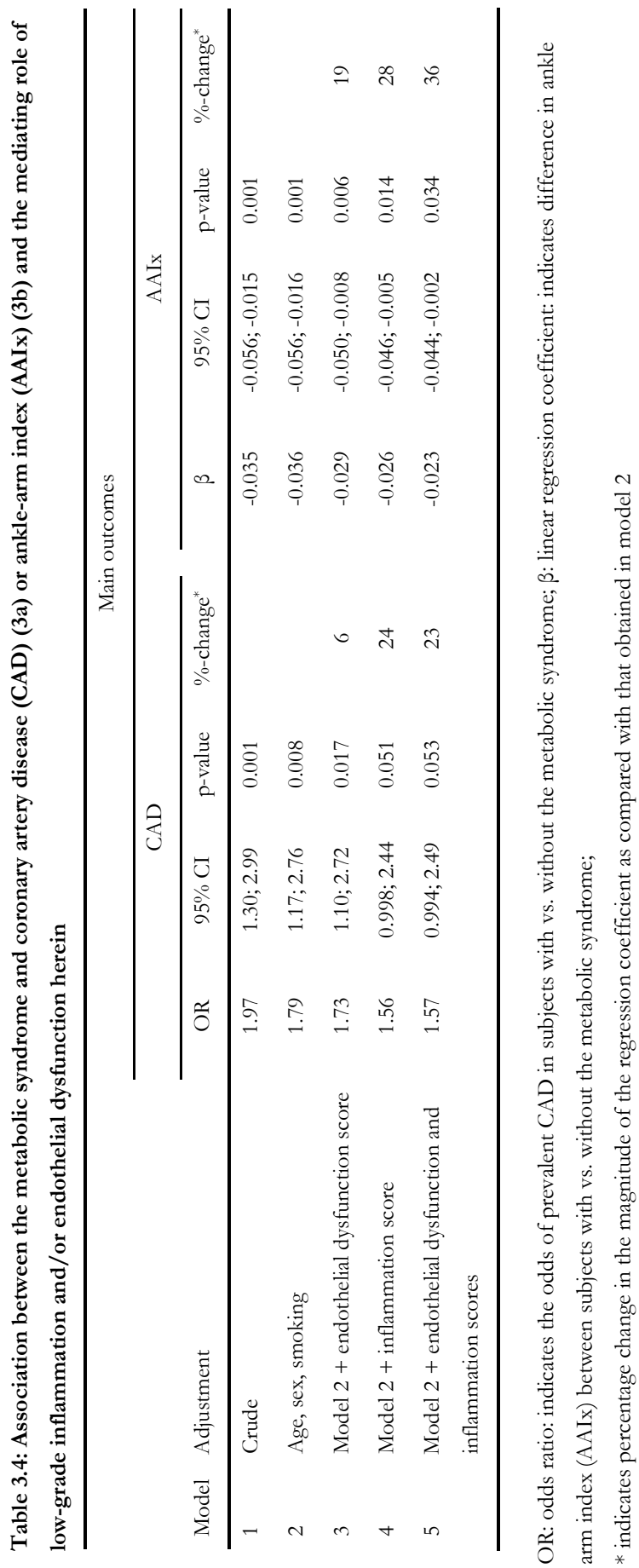




\subsubsection{Additional analyses}

Since the use of composite biomarkers is not a standard approach, we repeated our analyses using the individual markers of inflammation and endothelial dysfunction. The results of the combination of the separate markers was generally comparable to what was observed using the composite biomarkers; in addition to the 6 markers of inflammation, the 3 markers of endothelial dysfunction together mediated only a very limited part (i.e. $2.9 \%$ ) of the association between the metabolic syndrome and CAD, while mediation of the association between the metabolic syndrome and PAD was more pronounced (11.1\%). However, as mentioned before, this approach is less robust because of the problem of multiple testing ( 9 instead of 2 additional variables needed to be included in the analyses).

Classification of biomarkers as markers of inflammation or endothelial dysfunction may to some extent appear somewhat arbitrary. With respect to the markers we used in our current study, there is not much discussion in the literature in the classification of E-selectin and vWF as markers of endothelial dysfunction rather than inflammation [26] or the classification of CRP, IL6, SAA, ceruloplasmin and haptoglobin as markers of general inflammation rather than endothelial dysfunction. The classification of the cellular adhesion molecules may be more unequivocal. In our cohort, sICAM-1 was more strongly correlated to the markers of low-grade inflammation while sVCAM-1 was more strongly correlated with the markers of endothelial dysfunction (data not shown). Of note, when we repeated the analyses described herein, excluding sVCAM1 and sICAM-1 from the endothelial dysfunction and the low-grade inflammation scores, respectively, the results did not materially change: in addition to inflammation, endothelial dysfunction mediated only a very limited part (i.e. 1.5\%) of the association between the metabolic syndrome and CAD, while the additional mediation by endothelial dysfunction of the association between the metabolic syndrome and PAD was more pronounced $(16.7 \%)$.

As could be expected given the definition of the metabolic syndrome, the prevalence of T2DM differed between the subjects with and without the metabolic syndrome. However, when we repeated our final analyses with inclusion of T2DM as a covariate, mediation by endothelial dysfunction and low grade inflammation did not materially change from what was presented above (data not shown).

\subsection{Discussion}

We showed that endothelial dysfunction explained 19\% (8\% when additionally adjusted for low-grade inflammation) of the association between the metabolic syndrome and the severity of PAD, as measured by the AAIx. Part of this mediation was thus additional to low-grade inflammation. In contrast, endothelial dysfunction did not explain any part of the association between the metabolic syndrome and CAD. 
To our knowledge, the mediating role of endothelial dysfunction in addition to that of low-grade inflammation in the association between the metabolic syndrome and CAD or severity of PAD has not previously been described. Several studies have assessed the association of low-grade inflammation and/or endothelial dysfunction with either the metabolic syndrome $[3,5,9-11,30,31]$ or with CVD $[10,11,13,30,32,33]$, but these studies did not address their mediating role in the relation between the latter two. De Jager et al [32] did perform similar analyses as we present here, but their study investigated T2DM and did not focus on different aspects of macrovascular diseases. They showed that inflammation alone explained about $25 \%$ of the increase in cardiovascular mortalitiy risk conferred by T2DM while endothelial dysfunction and low-grade inflammation together explained about 43\% [32]. Wild et al recently described a mediating role of inflammation and hemostatic factors on the association between the metabolic syndrome and PAD[34]; and although the association between the metabolic syndrome and PAD was not significant in their analyses, the hazard ratio was attenuated by almost $10 \%$ when both inflammatory markers and hemostatic factors were included in the model.

In our current analyses, endothelial dysfunction was significantly associated with CAD in crude analyses, but the strength of this association was attenuated and statistical significance was lost after adjustment for age, sex and lifetime smoking. Previously published studies mostly used $\mathrm{vWF}$ as a marker of endothelial dysfunction. In line with our findings, several prospective studies reported significant associations between vWF and fatal and non-fatal coronary events [13, 35, 36] which were generally attenuated after adjustment for conventional risk factors. Only a limited number of mostly small studies investigated sE-selectin and/or sVCAM-1 as additional markers of endothelial dysfunction, but their associations with coronary events were less clear [37-39].

In contrast to the association with CAD, the association we observed between endothelial dysfunction and the severity of PAD remained significant after adjustment for age, sex and lifetime smoking. In the literature, only limited data are available on the association between endothelial dysfunction and the severity of PAD, and again most studies used vWF as marker of endothelial dysfunction. Various studies described an association between $\mathrm{vWF}$ and PAD (mostly defined as AAIx <0.9) [14, $33,40]$, while the association of $\mathrm{vWF}$ with the AAIx, as a measure of the severity of PAD, is less consistent [41-43]. Of note, our current data also show that the 28 subjects with PAD indeed had higher vWF levels than those without (data not shown).

An important strength of our study was the use of 6 biomarkers of low-grade inflammation (CRP, IL6, sICAM-1, SAA, ceruloplasmin and haptoglobin) and 3 for endothelial dysfunction (sE-selectin, sVCAM-1 and vWF), combined into two robust measures. Our current analyses showed that plasma levels of the markers for 
endothelial dysfunction were higher in subjects with CAD and with more severe PAD (crude associations, data not shown), similar to what we have shown before for markers of low-grade inflammation [12]. An important advantage of the use of $\mathrm{z}^{-}$ scores is that it is more robust than the separate markers and it limits the biological variation in the data. A potential disadvantage of the use of these biomarkers is that they provide an indirect measure of endothelial dysfunction in contrast to a functional test such as endothelium-dependent flow-mediated vasodilatation [44]. However, if biomarkers are a less powerful measure of endothelial dysfunction, the associations we found would underestimate the actual associations. So it might be that using a functional measure for endothelial dysfunction would result in stronger mediation by endothelial dysfunction. Additional limitations of our study include its cross-sectional design, which prohibits conclusions about causality. In other words, it cannot be excluded that part of the endothelial dysfunction was caused by, rather than was a cause of, PAD. Prospective studies are needed to further evaluate this. Since our study included a high-risk population, the extrapolation of our findings to other groups need to be made with caution. Lastly, we included only middle-aged Caucasian individuals, which prevents extrapolation of our results to populations in other age categories or with different ethnicities.

In conclusion, we showed that endothelial dysfunction explained part of the association between the metabolic syndrome and the severity of PAD in addition to low-grade inflammation, while it did not explain any of the association between the metabolic syndrome and CAD. This implies that inflammation and endothelial dysfunction do, at least not entirely, represent the same pathway in the relation between the metabolic syndrome and PAD, and that the pathogenesis of coronary and peripheral artery disease differ in this respect. Since inflammation and endothelial dysfunction explain only part of the association between the metabolic syndrome and cardiovascular disease other possible mechanisms/pathways, such as effects of inflammatory adipokines, coagulation and fibrinolysis or other hemodynamic mechanisms need to be investigated. Whether improvement of endothelial dysfunction can prevent metabolic-syndrome-associated peripheral arterial disease needs further investigation.

\subsection{References}

1 Alexander CM, Landsman PB, Teutsch SM and Haffner SM. NCEP-defined metabolic syndrome, diabetes, and prevalence of coronary heart disease among NHANES III participants age 50 years and older. Diabetes 2003;52:1210-4. metabolic syndrome and total and cardiovascular disease mortality in middle-aged men. Jama 2002;288:2709-16. 
Alberti KG, Zimmet P and Shaw J. The metabolic syndrome--a new worldwide definition. Lancet 2005;366:1059-62.

Kressel G, Trunz B, Bub A, Hulsmann O, Wolters M, Lichtinghagen R et al. Systemic and vascular markers of inflammation in relation to metabolic syndrome and insulin resistance in adults with elevated atherosclerosis risk. Atherosclerosis 2009;202:263-71.

Sjogren P, Basta G, de Caterina R, Rosell M, Basu S, Silveira A et al. Markers of endothelial activity are related to components of the metabolic syndrome, but not to circulating concentrations of the advanced glycation end-product $\mathrm{N}$ epsilon-carboxymethyl-lysine in healthy Swedish men. Atherosclerosis 2007;195:e168-75.

Alexander RW. Inflammation and coronary artery disease. N Engl J Med 1994;331:468-9.

Rizvi AA. Cytokine biomarkers, endothelial inflammation, and atherosclerosis in the metabolic syndrome: emerging concepts. Am J Med Sci 2009;338:310-8.

Ross R. The pathogenesis of atherosclerosis: a perspective for the 1990s. Nature 1993;362:8019. Brevetti G, Schiano V, Sirico G, Giugliano G, Laurenzano E and Chiariello M. Metabolic syndrome in peripheral arterial disease: relationship with severity of peripheral circulatory insufficiency, inflammatory status, and cardiovascular comorbidity. J Vasc Surg 2006;44:101-7; discussion 7.

Pischon T, Hu FB, Rexrode KM, Girman CJ, Manson JE and Rimm EB. Inflammation, the metabolic syndrome, and risk of coronary heart disease in women and men. Atherosclerosis 2008;197:392-9.

1 Rutter MK, Meigs JB, Sullivan LM, D'Agostino RB, Sr. and Wilson PW. C-reactive protein, the metabolic syndrome, and prediction of cardiovascular events in the Framingham Offspring Study. Circulation 2004;110:380-5.

2 Jacobs M, van Greevenbroek MM, van der Kallen CJ, Ferreira I, Blaak EE, Feskens EJ et al. Low-grade inflammation can partly explain the association between the metabolic syndrome and either coronary artery disease or severity of peripheral arterial disease: the CODAM study. Eur J Clin Invest 2009;39:437-44.

3 Danesh J, Wheeler JG, Hirschfield GM, Eda S, Eiriksdottir G, Rumley A et al. C-reactive protein and other circulating markers of inflammation in the prediction of coronary heart disease. N Engl J Med 2004;350:1387-97.

4 Wattanakit K, Folsom AR, Selvin E, Weatherley BD, Pankow JS, Brancati FL et al. Risk factors for peripheral arterial disease incidence in persons with diabetes: the Atherosclerosis Risk in Communities (ARIC) Study. Atherosclerosis 2005;180:389-97.

5 Ruotsalainen E, Vauhkonen I, Salmenniemi U, Pihlajamaki J, Punnonen K, Kainulainen S et al. Markers of endothelial dysfunction and low-grade inflammation are associated in the offspring of type 2 diabetic subjects. Atherosclerosis 2008;197:271-7.

Kruijshoop M, Feskens EJ, Blaak EE and de Bruin TW. Validation of capillary glucose measurements to detect glucose intolerance or type 2 diabetes mellitus in the general population. Clin Chim Acta 2004;341:33-40. 

association between abdominal adiposity and hyperglycemia. Diabetes Care 2001;24:1454-9. Grundy SM, Cleeman JI, Daniels SR, Donato KA, Eckel RH, Franklin BA et al. Diagnosis and management of the metabolic syndrome: an American Heart Association/National Heart, Lung, and Blood Institute Scientific Statement. Circulation 2005;112:2735-52. Wang Z, Ma J and Si D. Optimal cut-off values and population means of waist circumference in different populations. Nutr Res Rev 2010 july 20th :1-9 (doi:10.1017/S0954422410000120). Aboyans V, Criqui MH, Denenberg JO, Knoke JD, Ridker PM and Fronek A. Risk factors for progression of peripheral arterial disease in large and small vessels. Circulation 2006;113:26239.

McDermott MM, Green D, Greenland P, Liu K, Criqui MH, Chan C et al. Relation of levels of hemostatic factors and inflammatory markers to the ankle brachial index. Am J Cardiol 2003;92:194-9.

2 Atsma F, Bartelink ML, Grobbee DE and van der Schouw YT. Best reproducibility of the ankle-arm index was calculated using Doppler and dividing highest ankle pressure by highest arm pressure. J Clin Epidemiol 2005;58:1282-8. Gabay C and Kushner I. Acute-phase proteins and other systemic responses to inflammation. N Engl J Med 1999;340:448-54. Shoelson SE, Lee J and Goldfine AB. Inflammation and insulin resistance. J Clin Invest 2006;116:1793-801.

Al-Isa AN, Thalib L and Akanji AO. Circulating markers of inflammation and endothelial dysfunction in Arab adolescent subjects: Reference ranges and associations with age, gender, body mass and insulin sensitivity. Atherosclerosis 2009.

Freestone B, Chong AY, Nuttall S and Lip GY. Impaired flow mediated dilatation as evidence of endothelial dysfunction in chronic atrial fibrillation: relationship to plasma von Willebrand factor and soluble E-selectin levels. Thromb Res 2008;122:85-90. Stehouwer CD. Is measurement of endothelial dysfunction clinically useful? Eur J Clin Invest 1999;29:459-61.

28 Stam F, van Guldener C, Schalkwijk CG, ter Wee PM, Donker AJ and Stehouwer CD. Impaired renal function is associated with markers of endothelial dysfunction and increased inflammatory activity. Nephrol Dial Transplant 2003;18:892-8.

29 Schram MT, Chaturvedi N, Schalkwijk C, Giorgino F, Ebeling P, Fuller JH et al. Vascular risk factors and markers of endothelial function as determinants of inflammatory markers in type 1 diabetes: the EURODIAB Prospective Complications Study. Diabetes Care 2003;26:2165-73. Langenberg C, Bergstrom J, Scheidt-Nave C, Pfeilschifter J and Barrett-Connor E. Cardiovascular death and the metabolic syndrome: role of adiposity-signaling hormones and inflammatory markers. Diabetes Care 2006;29:1363-9.

31 Olijhoek JK, van der Graaf Y, Banga JD, Algra A, Rabelink TJ and Visseren FL. The metabolic syndrome is associated with advanced vascular damage in patients with coronary heart disease, stroke, peripheral arterial disease or abdominal aortic aneurysm. Eur Heart J 2004;25:342-8. 
de Jager J, Dekker JM, Kooy A, Kostense PJ, Nijpels G, Heine RJ et al. Endothelial dysfunction and low-grade inflammation explain much of the excess cardiovascular mortality in individuals with type 2 diabetes: the Hoorn Study. Arterioscler Thromb Vasc Biol 2006;26:1086-93.

Reich LM, Heiss G, Boland LL, Hirsch AT, Wu K and Folsom AR. Ankle-brachial index and hemostatic markers in the Atherosclerosis Risk in Communities (ARIC) study cohort. Vasc Med 2007;12:267-73.

Wild SH, Byrne CD, Tzoulaki I, Lee AJ, Rumley A, Lowe GD et al. Metabolic syndrome, haemostatic and inflammatory markers, cerebrovascular and peripheral arterial disease: The Edinburgh Artery Study. Atherosclerosis 2009;203:604-9. Morange PE, Bickel C, Nicaud V, Schnabel R, Rupprecht HJ, Peetz D et al. Haemostatic factors and the risk of cardiovascular death in patients with coronary artery disease: the AtheroGene study. Arterioscler Thromb Vasc Biol 2006;26:2793-9. Whincup PH, Danesh J, Walker M, Lennon L, Thomson A, Appleby P et al. von Willebrand factor and coronary heart disease: prospective study and meta-analysis. Eur Heart J 2002;23:1764-70.

Anderson R, Dart AM, Starr J, Shaw J and Chin-Dusting JP. Plasma C-reactive protein, but not protein S, VCAM-1, von Willebrand factor or P-selectin, is associated with endothelium dysfunction in coronary artery disease. Atherosclerosis 2004;172:345-51.

Ruef J, Marz W and Winkelmann BR. Markers for endothelial dysfunction, but not markers for oxidative stress correlate with classical risk factors and the severity of coronary artery disease. (A subgroup analysis from the Ludwigshafen Risk and Cardiovascular Health Study). Scand Cardiovasc J 2006;40:274-9.

39 Shai I, Pischon T, Hu FB, Ascherio A, Rifai N and Rimm EB. Soluble intercellular adhesion molecules, soluble vascular cell adhesion molecules, and risk of coronary heart disease. Obesity (Silver Spring) 2006;14:2099-106.

40 Makin AJ, Chung NA, Silverman SH and Lip GY. Thrombogenesis and endothelial damage/dysfunction in peripheral artery disease. Relationship to ethnicity and disease severity. Thromb Res 2003;111:221-6.

41 Blann AD, Farrell A, Picton A and McCollum CN. Relationship between endothelial cell markers and arterial stenosis in peripheral and carotid artery disease. Thromb Res 2000;97:20916.

42 Khaleghi M, Singletary LA, Kondragunta V, Bailey KR, Turner ST, Mosley TH, Jr. et al. Haemostatic markers are associated with measures of vascular disease in adults with hypertension. J Hum Hypertens 2009.

43 Tzoulaki I, Murray GD, Price JF, Smith FB, Lee AJ, Rumley A et al. Hemostatic factors, inflammatory markers, and progressive peripheral atherosclerosis: the Edinburgh Artery Study. Am J Epidemiol 2006;163:334-41. 
44 Henry RM, Ferreira I, Dekker JM, Nijpels G, Scheffer PG and Stehouwer CD. The metabolic syndrome in elderly individuals is associated with greater muscular, but not elastic arterial stiffness, independent of low-grade inflammation, endothelial dysfunction or insulin resistanceThe Hoorn Study. J Hum Hypertens 2009. 


\section{Chapter 4}

\section{Human plasma complement C3 is independently associated with coronary heart disease, but only in heavy smokers (the CODAM study)}




\begin{abstract}
Background: Complement C3 is an emerging risk factor for coronary heart disease (CHD) and is particularly increased in the metabolic syndrome. A direct effect of smoking on structure and function of complement C3 has been suggested. Hypothesis: Smoking behaviour may affect the cardiovascular risk that is associated with plasma complement C3. Methods: The association between plasma C3 and CHD was studied in the CODAM (Cohort on Diabetes and Atherosclerosis Maastricht) study population ( $\mathrm{n}=562,61 \%$ male) with examination of effect modification by smoking. Results: The overall prevalence of CHD was $23.3 \%$. Higher plasma C3 levels were associated with a higher CHD prevalence, and there was a significant interaction with heavy smoking $(p=0.01)$. In never $\&$ light smokers, the univariate $O R$ for CHD per 1 s.d. (0.33 g/L) increase in C3 was 1.09 (95\% confidence interval (CI) 0.85-1.41) $(\mathrm{p}=0.505)$ whereas in heavy smokers it was $2.05(1.43-2.93)(\mathrm{p}<0.001)$. The association in the group of heavy smokers remained significant (OR 2.38 (1.54-3.68), $\mathrm{p}<0.001$ ) after adjustment for traditional risk factors for cardiovascular disease and also after further adjustment for other cardiometabolic risk factors, i.e. the metabolic syndrome, CRP and insulin resistance (HOMA2IR) (OR C3 between 2.16 and 2.29, all $\mathrm{p} \leq 0.001$ ). In conclusion: Human plasma complement $\mathrm{C} 3$ is associated with prevalent CHD, but only in heavy smokers, and this association is independent of important metabolic cardiovascular risk factors.
\end{abstract}




\subsection{Introduction}

Coronary heart disease (CHD) is increasingly perceived as a disease of the immune system because of the low-grade inflammation that is seen in individuals with established cardiovascular disease and in those at increased risk of CHD including individuals with the metabolic syndrome or type 2 diabetes (T2DM). The complement system is the major effector of the humeral arm of the immune system. Its third component (C3) plays a pivotal role in the complement cascade and is the most abundant complement protein in the circulation. Complement $\mathrm{C} 3$ is an emerging risk factor for CHD [1] and higher complement C3 levels predicted a higher risk of new CHD events in women who had received aorto-coronary bypass graft surgery [2]. Complement C3 is particularly increased in disorders that are characterized by insulin resistance such as T2DM and the metabolic syndrome [3-7]. The association between C3 and CHD has been reported to be independent of smoking, hypertension, cholesterol and diabetes [8-10], although recent data from a large prospective study suggested that it may not be independent of these CVD risk factors [11]. The exact nature of the relation between high plasma complement $\mathrm{C} 3$ levels and the prevalence of CHD remains to be clarified.

We hypothesized that another strong risk factor for cardiovascular disease, i.e. smoking, may affect the cardiovascular risk associated with high complement C3 levels. It has been shown that, at least in vitro, cigarette smoke can activate the alternative pathway of complement by modifying complement C3 [12-15]. In addition, tobacco glycoprotein can activate the classical complement pathway, possibly via direct interaction with complement component C1Q [16]. These effects of cigarette smoke could, theoretically, result in the generation of biologically active complement cleavage products and thus enhance the effect of complement on the risk of cardiovascular disease.

In view of these considerations, we hypothesized that the expected association between complement C3 and CHD would not be the same in individuals with different smoking behaviour, i.e. that there would be effect modification of smoking and plasma complement $\mathrm{C} 3$ on the prevalence of CHD. We also evaluated whether this relation was confounded and/or mediated by known cardiometabolic risk factors such as the metabolic syndrome, inflammation and insulin resistance.

\subsection{Methods}

\subsubsection{Study population}

The CODAM cohort $(n=574)$ has been described in detail previously $[17 ; 18]$. In short, participants were selected from a large population-based cohort [19]. Inclusion 
criteria were Caucasian descent and age $>40$ years and, in addition, at least one of the following criteria: body mass index $(B M I)>25 \mathrm{~kg} / \mathrm{m}^{2}$; a positive family history of T2DM; a history of gestational diabetes and/or glucosuria; and use of antihypertensive medication. Combinations of these separate inclusion criteria were also allowed. Subjects were excluded if they had missing data on one or more of the biochemical and clinical markers that were used in the analyses. The present study population therefore consisted of 562 individuals. The study was approved by the local Medical Ethical Committee of the Maastricht University and the University Hospital. All individuals gave written informed consent.

\subsubsection{Individual characterization}

Individuals were asked to stop their lipid-lowering medication 14 days prior to blood withdrawal. According the results of a standard $75 \mathrm{~g}$ oral glucose tolerance test, 141 subjects had T2DM [20]. Of these T2DM subjects, 54\% were not aware of their diabetes status prior to the screening. BMI $\left(\mathrm{kg} / \mathrm{m}^{2}\right)$ was calculated from weight $(\mathrm{kg})$ without shoes and wearing light indoor clothing and height $(\mathrm{m})$. Waist was measured at the level midway between the lateral lower rib margin and the spina iliaca anterior superior. Blood pressure was measured twice on each visit after 5 minutes of rest with an oscillometric precision blood pressure instrument (Maxi stable 3, Speidel \& Keller) on the right arm in the supine position. Presence of the metabolic syndrome was determined according to the National Cholesterol Education Program (NCEP) (Adult Treatment Panel (ATP) III definition as modified by the American Heart Association (AHA) / National Heart, Lung and Blood Institute (NHLBI) [21].

The main outcome of this study, coronary heart disease (CHD), was defined as the occurrence of previous myocardial infarction, bypass of the coronary arteries, percutaneous coronary intervention (ascertained by questionnaire) and/or the presence of signs of myocardial infarction (Minnesota codes 1-1 or 1-2) or ischemia (Minnesota codes 1-3, 4-1, 4-2, 4-3, 5-1, 5-2, 5-3 or 7-1) on a 12-lead ECG. For the major part of the cases $(>75 \%$ of individuals with $\mathrm{CHD})$, the self-reports could be confirmed using available hospital registries.

Smoking habits were determined with the help of an extensive questionnaire about current tobacco smoking, smoking in the past, and quitting attempts. Individuals were categorized into three groups: never, light and heavy smokers. Light smokers were defined as former or current smokers who smoked/smoke less than or equal to the median pack-years of cigars or cigarettes (one pack-year is $20 \mathrm{~g}$ of tobacco per day, smoked over a course of 1 year). Current or previous tobacco consumption by the former \& current light smokers was 12.5 (7.0-17.8) (median (interquartile range)) pack-years. Heavy smokers are former \& current smokers who smoked/smoke more than the median amount of pack-years (tobacco consumption by heavy smokers was 39.0 (30.2-53.3) pack-years). It could not be assessed with certainty whether former 
smokers have stopped smoking prior to or after their first event, since we did not obtain information on the year of the first cardiovascular event. In our current crosssectional analyses we assumed that a substantial part of the group of former smokers had stopped smoking after the cardiovascular event. For this reason, former and current smokers were collapsed into one group. We verified this assumption by calculating the cross-product of current smoking and plasma C3.

\subsubsection{Biochemical analyses}

After an overnight fast (12-14 hrs), venous blood was collected in pre-cooled EDTA vacutainer tubes. After centrifugation at $3600 \mathrm{~g}$ for $15 \mathrm{~min}$ at $4^{\circ} \mathrm{C}$, plasma aliquots were stored at $-80^{\circ} \mathrm{C}$ until use. All samples were thawed only once prior to plasma measurements. The main predictor in this study, complement C3 was measured in serum by autoanalyzer (Hitachi 912) using a Roche kit assays. The interassay variation of the C3 determination was $2.14 \%$ and the average C3 concentration of the reference serum was exactly as reported by the manufacturer (protein Precinorm; C3, $1.36 \mathrm{~g} / \mathrm{L}$ ). C-reactive protein (CRP) was also measured in serum using the CRP-Latex assay (Roche diagnostics). The following variables were determined in EDTA plasma; HDL-cholesterol using the HDL-C plus assay, cholesterol using the CHOD-PAP assay, triglycerides using the TG GPO-PAP assay. Plasma insulin concentrations were determined with a two-sided immunoradiometric test, using paired monoclonal antibodies (Medgenix Diagnostics). Glucose concentrations were measured in $\mathrm{NaF} / \mathrm{KOx}$ plasma with the hexokinase glucose-6-phosphate dehydrogenase method (HK-G6PD method; ABX Diagnostics Glucose HK 125). Insulin sensitivity (homeostasis model assessment (HOMA)-insulin resistance (IR)) was estimated using the HOMA2 calculator (www.dtu.ox.ac.uk) based on the nonlinear computer model [22].

\subsubsection{Statistical analyses}

Variables with a normal distribution are presented as mean \pm standard deviation (SD). Variables with skewed distribution (i.e. insulin, HOMA2-IR, CRP and triglycerides) were log-transformed prior to further analyses. These variables are presented as median (interquartile range). Comparisons between different groups were assessed by Student's t-test (continuous data) or $\chi^{2}$ tests (frequency data). A two-sided P-value $<0.05$ indicated statistical significance.

In the logistic regression analyses, complement C3 was converted to a Z-score (unit is standard deviation, SD) which allowed us to present the main results of the logistic regression analyses as the change in OR for one SD increase in C3. First, logistic regression analyses were performed to analyse the association between C3 and CHD. Effect modification by smoking was investigated by adding interaction terms (the 
cross-product of smoking and plasma C3) to the logistic regression model. When significant interaction $(\mathrm{p}<0.1)$ was present, we proceeded with stratified analyses with individuals divided into groups according to smoking behaviour. Analyses were performed with adjustments for age, sex, BMI, total cholesterol and T2DM $(\mathrm{Y} / \mathrm{N})$. Next, we investigated whether the observed associations were independent of cardiometabolic risk factors i.e. the metabolic syndrome, low-grade inflammation (as represented by CRP) and insulin resistance (HOMA2-IR). Use of antihypertensive and glucose-lowering medication were not included as covariates since these are included in the definition of the metabolic syndrome.

All analyses were done using the Statistical Package for the Social Sciences (SPSS) version 15.0 for windows (SPSS Inc, Chicago IL, USA).

\subsection{Results}

In the whole population, plasma $\mathrm{C} 3$ concentration was significantly associated with a higher prevalence of CHD: the odds ratio (OR) for CHD per $1 \mathrm{SD}(0.33 \mathrm{~g} / \mathrm{l})$ increase in C3 was 1.39 (95\% CI: 1.14-1.68), $\mathrm{p}=0.001$. This association remained significant (OR C3, 1.50 (1.18-1.90), $\mathrm{p}=0.001$ ) after adjustment for relevant cardiovascular risk factors i.e. sex, age, BMI, cholesterol, T2DM and smoking behaviour (never, light or heavy smoker).

Basic characteristics of the study population stratified by smoking behaviour are presented in Table 4.1. Addition of cross-products of smoking behaviour and plasma C3 to the logistic regression model revealed interaction between C3 and heavy smoking on the risk of prevalent CHD ( $p$ for interaction $=0.01$ ), while there was no indication of interaction between $\mathrm{C} 3$ and light smoking ( $\mathrm{p}$ for interaction $=0.51$ ). Next, subjects were stratified into two groups; a group of heavy smokers and a group of light \& never smokers. Addition of the cross-product of light smoking and plasma C3 to the logistic regression analyses of the light \& never smokers again showed that there was no interaction between C3 and light smoking ( $\mathrm{p}$ for interaction $=0.49$ ). For this reason, further analyses were done in the "never" and "light" smokers combined into one group ( $n=366 ; 71$ CHD cases) and in the group of heavy smokers $(n=196 ; 60$ CHD cases). In never \& light smokers, the univariate OR for CHD per 1 SD increase in C3 was $1.09(0.85-1.41)(\mathrm{p}=0.505)$ whereas in heavy smokers it was $2.05(1.43-2.93)$ $(\mathrm{p}<0.001$, Table 4.2, model 1), and remained significant (OR 2.38 (1.54-3.68), $\mathrm{p}<0.001$, Table 4.2, model 2) after adjustment for traditional risk factors for cardiovascular disease (sex, age, total cholesterol, T2DM). For completion, separate analyses were done to confirm that the association between $\mathrm{C} 3$ and CHD in subjects who never smoked ( $\mathrm{n}=165,25 \mathrm{CHD}$ cases, OR $1.28(0.78 ; 2.09), \mathrm{p}=0.33)$ and in the former \& current light smokers $(n=201,46$ CHD cases, OR $1.04(0.67 ; 1.58), p=0.84)$ was indeed comparable (as already indicated by the non-significant cross-product of 
light smoking and C3 as shown above). To substantiate our decision to combine the former \& current smokers into one group, we also investigated the association in the former and current heavy smokers separately, and the adjusted OR's for CHD per 1 $\mathrm{SD}$ increase in C3 in these analyses were 2.34 (1.32-1.14) (35 CHD cases, $\mathrm{p}=0.004$ ) and 2.53 (1.24-5.13) (25 CHD cases, $\mathrm{p}=0.010$ ), respectively. The complete absence of interaction between current smoking and C3 on the prevalence of CHD ( $p$ for the cross-product $=0.75$ ) indicated that these OR's did not differ significantly. Taken together, a 1 SD increase in plasma C3 was associated with a 2 to 2.5-fold higher prevalence of CHD in heavy smokers, while in the never and light smokers a similar increase in C3 was essentially not associated with a higher prevalence of CHD.

The heavy smokers, especially those with CHD, had besides higher C3 levels also more other cardiometabolic risk factors such as the metabolic syndrome, insulin resistance and low-grade inflammation (Table 4.1) and from various human studies, plasma C3 levels are known to be associated with these particular risk factors [3-7]. We therefore investigated whether the association between plasma complement C3 and CHD in heavy smokers was independent of these cardiometabolic risk factors. When the metabolic syndrome was included in the model, the OR for C3 in the heavy smokers decreased only slightly, from 2.38 (1.54-3.68) to 2.19 (1.40-3.43) (Table 4.2, model $2 \& 3)$. Likewise, after additional adjustments for inflammation and insulin resistance the OR's were 2.29 (1.43-3.65) and 2.16 (1.37-3.40), respectively (Table 4.2, model $4 \& 5$ ). The association between $\mathrm{C} 3$ and CHD in heavy smoking also remained significant when all metabolic cardiovascular risk factors that were addressed were included simultaneously (Table 4.2, model 6). The association between C3 and CHD in the group of never \& light smokers was and remained non-significant and very modest, if present at all (Table 4.2, models 1-6). There are indications in the literature that statin therapy might reduce plasma levels of complement C3 (23-25) and since a substantial number of subjects in our study received statin therapy $(42 \%$ of the subjects with $\mathrm{CHD}, 11 \%$ of those without) this might to some extent have confounded our results. With additional adjustment of the full model in Table 4.2. (model 6) for statin therapy, the OR for C3 remained $1.73(1.03 ; 2.92)(p=0.04)$. 


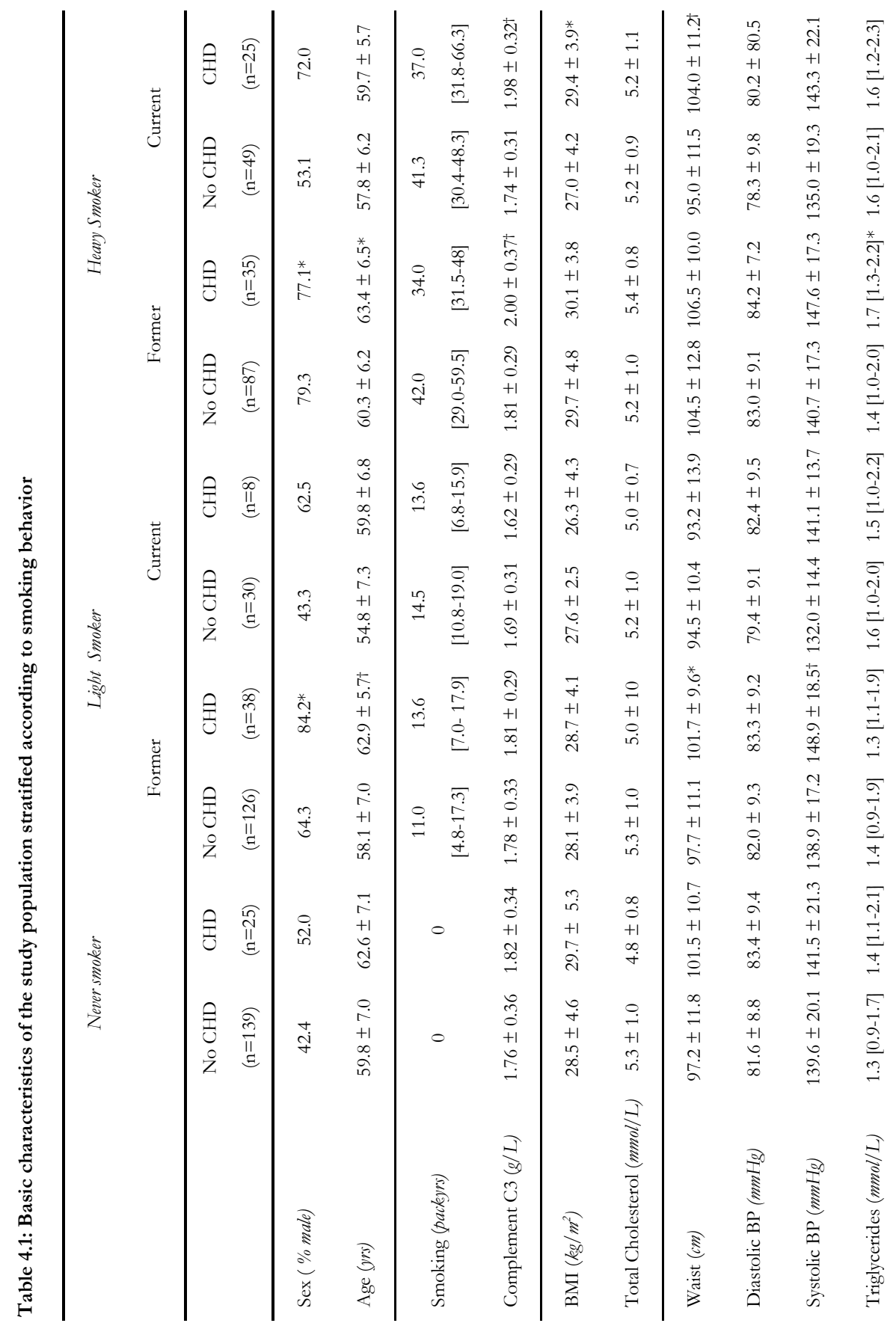




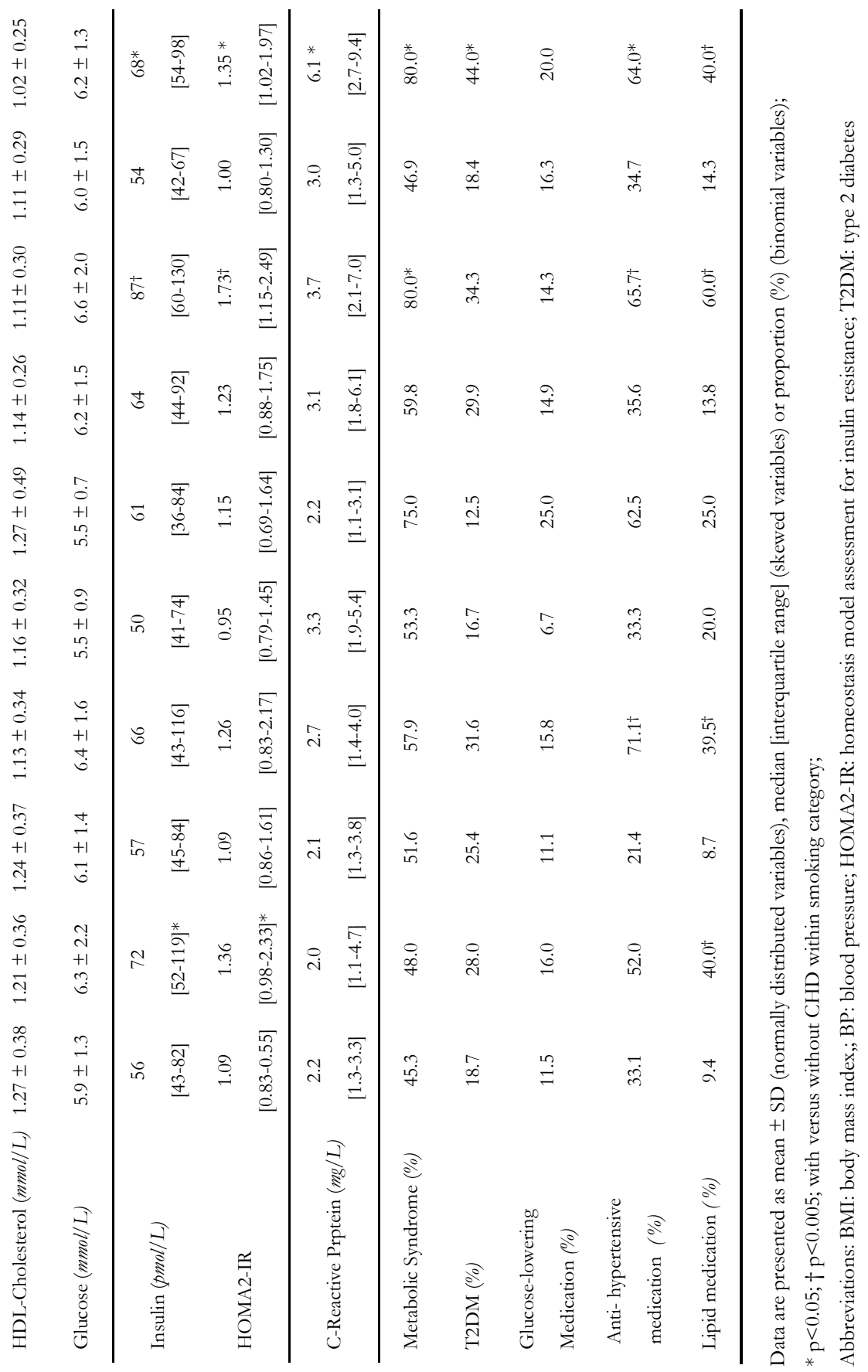




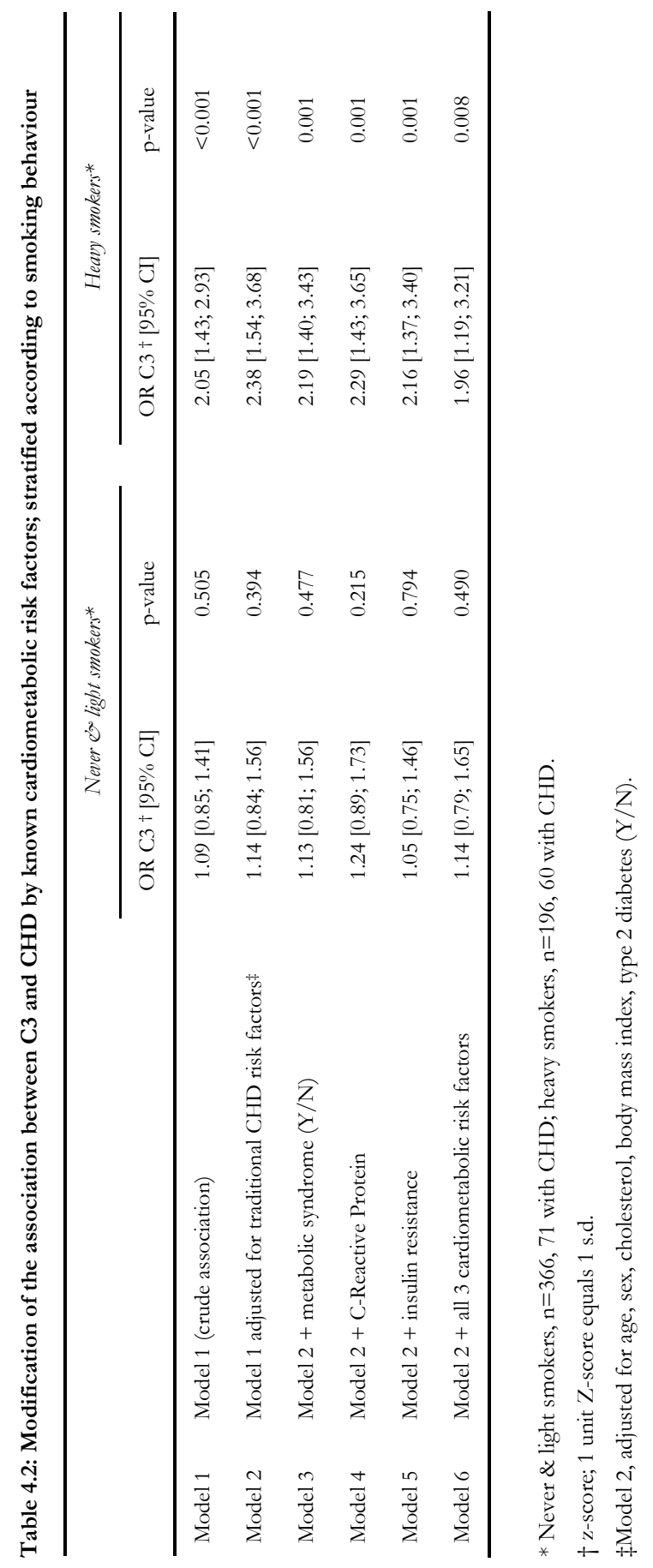




\subsection{Discussion}

The main conclusion of our current study is that human plasma complement C3 was indeed associated with prevalent CHD in our whole study population, but careful examination learned that this relation was only present in the group of heavy smokers. The association between C3 and prevalence of CHD was independent of cardiometabolic risk factors such as insulin resistance, systemic inflammation, and the metabolic syndrome.

Complement $\mathrm{C} 3$ has been established as a cardiovascular risk associate in both crosssectional and in longitudinal studies. It is often regarded as a component of inflammatory activity and is also strongly associated with insulin resistance. Our findings of an overall association between C3 and CHD are in keeping with several studies that reported associations between $\mathrm{C} 3$ and prevalence or incidence of cardiovascular disease, independent of age, sex and a limited number of additional risk factors $[1 ; 2 ; 8 ; 26]$. We have extended those currently available data by showing a strong effect-modification of smoking behaviour on the association between C3 and CHD. The association was, in fact, only present in the group of heavy smokers. This leads us to suggest that the previously-reported associations between C3 and CHD may, in fact, have also been restricted to individuals who were heavy smokers which raises the possibility that in these reports, the actual effect of C3 in specific risk groups may have been diluted. Moreover, part of the variation on the reported associations may be related to the relative number of smokers in the research populations.

Our hypothesis that there may be effect-modification between smoking and C3 on prevalence of CHD was based on several papers that showed a direct effect of cigarette smoke, or constituents of cigarette smoke, on the structure and function of complement components, including C3 [12;13;16]. These effects of cigarette smoke could, theoretically, result in the generation of biologically active complement cleavage products and thus enhance the effect of complement on the risk of cardiovascular disease. Such an activation would not necessarily lead to a measurable consumption of C3 since the plasma concentration of $\mathrm{C} 3$ exceeds that of its activation products by $>1000$-fold [27;28]. In our view, activation of complement C3 (in subjects with high C3 levels) by (heavy) smoking may be one of the mechanisms by which smoking can enhance the inflammatory process in the atherosclerotic plaque and thereby affect plaque composition and vulnerability and increase the risk of CVD. An alternative explanation for our observations could be that smoking would not directly affect (activation of) the complement C3 protein itself, but rather that (heavy) smoking would induce oxidative stress and inflammatory processes in the plaque which might then affect the expression of complement regulators to an extent that it would no longer suffice to control complement activation, especially in those subjects with high complement C3 levels. Since we have used cross-sectional, observational data in our 
current analyses, we can presently not differentiate between these alternative explanations and longitudinal and experimental data are needed for a final conclusion. It remains to be conclusively determined whether $\mathrm{C} 3$ is actually a causal player in the development of CHD, or rather an innocent bystander reflecting a yet unknown metabolic mechanism. Our current data suggest a causal relation in which structural changes in complement $\mathrm{C} 3$, induced by heavy smoking, may accelerate the atherosclerotic process, but only if plasma C3 concentrations are relatively high. Such an effect of cigarette smoke on complement C3 may take place at various distinct locations such as in the circulation, the lungs, or locally in the atherosclerotic plaque. A growing body of evidence suggests a role for C3 activation in plaque formation or progression, which implies that not $\mathrm{C} 3$ by itself, but rather its activation products $\mathrm{C} 3 \mathrm{a}$ and $\mathrm{C} 3 \mathrm{~b}$, which are generated upon cleavage of $\mathrm{C} 3$, may be involved in the atherothrombotic process. Association of complement factor $\mathrm{C} 3 \mathrm{~b}$ with lipid components in the vessel wall [29-31] may induce an atherosclerotic response and where no significant complement activation takes place in normal arteries, complement is extensively activated in atherosclerotic lesions and is especially strong in vulnerable and ruptured plaques [32-35]. Moreover, inhibition of C3 activation reduces vein graft atherosclerosis in apoE3-Leiden transgenic mice [36]. In addition, Kostner et al showed in a small group of patients that C3a may be higher in unstable than in stable angina pectoris [37].

In this study we have identified a subgroup group of heavy smokers, i.e. those with high C3 levels, who have a particularly high risk of CHD. Note that we specifically addressed the question whether smoking would affect (i.e. strengthen) the association between high levels of C3 and CHD. We did not hypothesize or address the question whether (heavy) smoking would somehow lead to higher plasma C3 concentrations. Nevertheless, plasma levels of C3 in the former and current heavy smokers without CHD were similar to those in subjects who never smoked (Table 4.1). Indeed, linear regression analyses with adjustment for age, sex, T2DM, total cholesterol and BMI showed that in these subjects plasma C3 levels in former and current heavy smokers did not differ from those in subjects who never smoked (data not shown), which strongly suggests that the higher C3 levels in the heavy smokers with CHD are not caused by the smoking habit itself. Hence it is rather the combination of the presence of high C3 levels and the habit of heavy smoking that underlies an increased risk of CHD in those subjects.

Potential limitations of our study lie in the fact that the CODAM population consisted of middle-aged Caucasian individuals with an increased T2DM and CHD risk profile and thus did not represent the general population. Also, given the cross-sectional design of our study, we cannot completely rule out the possibility that the association between $\mathrm{C} 3$ and $\mathrm{CHD}$ may in fact be caused by metabolic changes that can be induced in response to a myocardial infarction. However, we consider this highly 
unlikely since in the group consisting of never \& light smokers, plasma C3 concentration did not differ between individuals with CHD and those without (Table 4.1). Moreover, both prospective human data [2] as well as animal studies suggest a causal relation [36]. Smoking cessation is associated with a pronounced increase in body weight and since plasma C3 concentration is associated with obesity one could argue that our current findings may to some extent be related to changes in body weight due to smoking cessation after a cardiovascular event. However, plasma C3 levels are consistently higher in the subjects with CHD, both in the former and in the current smokers, irrespective of the fact that the former smokers were indeed more obese, and in this group obesity did not differ between those with and without CHD (Table 4.1). This strongly suggests that the higher C3 levels in the heavy smokers with CHD are not due to increased body weight after smoking cessation. The plasma levels of complement $\mathrm{C} 3$ in the CODAM population are relatively high compared to some other studies [6;38]. This may be at least partly related to the fact that the CODAM subjects do not comprise a random sample of the general population, but are in fact selected for an increased risk of diabetes and cardiovascular disease. However, it is unlikely that these relatively high levels will have compromised our results since we obtained a low interassay coefficient of variation $(2.14 \%)$, all samples were analyzed in a blinded fashion and all comparisons were made within the CODAM study population.

In conclusion, we showed a cross-sectional association between human plasma complement C3 and prevalent CHD, but only in heavy smokers. This association was independent of systemic inflammation, insulin resistance and the metabolic syndrome. This independent association may be related to a direct effect of smoking on complement C3 or to other, yet to be identified mechanisms that may lead to complement activation in this particular group of subjects. To further pin-point whether this observed interaction between heavy smoking and complement C3 on $\mathrm{CHD}$ is indeed generalizable to other populations, it may be worthwhile to re-analyse the relation between $\mathrm{C} 3$ and CHD in previously-published cohorts with focus on interaction with smoking habits.

\subsection{Reference list}

1. Muscari A, Bozzoli C, Puddu GM et al. Association of serum C3 levels with the risk of myocardial infarction. Am J Med. 1995; 98:357-64.

2. Szeplaki G, Prohaszka Z, Duba J et al. Association of high serum concentration of the third component of complement (C3) with pre-existing severe coronary artery disease and new vascular events in women. Atherosclerosis. 2004; 177:383-9.

3. Engström G, Hedblad B, Eriksson KF, Janzon L, Lindgärde F. Complement C3 is a risk factor for the development of diabetes: a population-based cohort study. Diabetes 2005; 54:570-5. 
4. Hernández-Mijares A, Jarabo-Bueno MM, López-Ruiz A, Solá-Izquierdo E, Morillas-Ariño C, Martínez-Triguero ML. Levels of C3 in patients with severe, morbid and extreme obesity: its relationship to insulin resistance and different cardiovascular risk factors. Int J Obes. 2007; 6:927-32.

5. van Oostrom AJ, Alipour A, Plokker TW, Sniderman AD, Cabezas MC. The metabolic syndrome in relation to complement component 3 and postprandial lipemia in patients from an outpatient lipid clinic and healthy volunteers. Atherosclerosis. 2007; 190:167-73.

6. Muscari A, Antonelli S, Bianchi G et al. Serum C3 is a stronger inflammatory marker of insulin resistance than $\mathrm{C}$-reactive protein, leukocyte count, and erythrocyte sedimentation rate: comparison study in an elderly population. Diabetes Care. 2007; 30:2362-8.

7. Engström G, Hedblad B, Janzon L, Lindgärde F. Weight gain in relation to plasma levels of complement factor 3: results from a population-based cohort study. Diabetologia 2005; 48:2525-31.

8. Ajjan R, Grant PJ, Futers TS et al. Complement C3 and C-reactive protein levels in patients with stable coronary artery disease. Thromb Haemost. 2005;94:1048-53.

9. Onat A, Uzunlar B, Hergenç G et al. Cross-sectional study of complement C3 as a coronary risk factor among men and women. Clin Sci (Lond). 2005; 108:129-35.

10. Carter AM, Prasad UK, Grant PJ. Complement C3 and C-reactive protein in male survivors of myocardial infarction. Atherosclerosis. 2009; 203:538-43.

11. Engström G, Hedblad B, Janzon L, Lindgärde F. Complement C3 and C4 in plasma and incidence of myocardial infarction and stroke: a population-based cohort study. Eur J Cardiovasc Prev Rehabil. 2007; 14:392-7.

12. Kew RR, Ghebrehiwet B, Janoff A. Cigarette smoke can activate the alternative pathway of complement in vitro by modifying the third component of complement. J Clin Invest. 1985; 75:1000-7.

13. Kew RR., Ghebrehiwet B, Janoff A. Characterization of the third component of complement (C3) after activation by cigarette smoke. Clin Immunol Immunopathol. 1987; 44:248-58.

14. Robbins RA, Nelson KJ, Gossman GL, Koyama S, Rennard SI. Complement activation by cigarette smoke. Am J Physiol. 1991; 260:L254-L259.

15. Perricone R, de Carolis C, de Sanctis G, Fontana L. Complement activation by cigarette smoke condensate and tobacco infusion. Arch Environ Health. 1983; 38:176-9.

16. Koethe SM, Nelson KE, Becker CG. Activation of the classical pathway of complement by tobacco glycoprotein (TGP). J Immunol. 1995; 155:826-35.

17. Corpeleijn E, van der Kallen CJ, Kruijshoop M et al. Direct association of a promoter polymorphism in the CD36/FAT fatty acid transporter gene with Type 2 diabetes mellitus and insulin resistance. Diabet Med. 2006; 23:907-11.

18. Kruijshoop M, Feskens EJ, Blaak EE, de Bruin TW. Validation of capillary glucose measurements to detect glucose intolerance or type 2 diabetes mellitus in the general population. Clin Chim Acta.2004; 341:33-40. 
19. van Dam RM, Boer JM, Feskens EJ, Seidell JC. Parental history of diabetes modifies the association between abdominal adiposity and hyperglycemia. Diabetes Care. 2001; 24:1454-9.

20. World Health Organization. Definition, diagnosis and classification of Diabetes Mellitus ans its complication. In: Report af a WHO Consultation. Part 1: Diagnosis and classification of Diabetes Mellitus. WHO Technical Report Series. 1999; 1-59.

21. Grundy SM, Cleeman JI, Daniels SR et al. Diagnosis and management of the metabolic syndrome: an American Heart Association/National Heart, Lung, and Blood Institute Scientific Statement. Circulation 2005; 112:2735-52.

22. Levy J, Matthews DR, Hermans MP. Correct homeostasis model assessment (HOMA) evaluation uses the computer program (Letter). Diabetes Care 1998; 21:2191-2.

23. Muscari A, Bastagi L, Poggiopollini G et al. Short term effect of atorvastatin and vitamin E on serum levels of $\mathrm{C} 3$, a sensitive marker of the risk of myocardial infarction in men. Cardiovasc Drugs Ther. 2001; 15:453-8.

24. Di Garbo V, Bono M, Di Raimondo D, De Simone R, Raneli G, Avellone G. Non lipid, dosedependent effects of pravastatin treatment on hemostatic system and inflammatory response. Eur J Clin Pharmacol. 2000; 56:277-84.

25. Verseyden C, Meijssen S, van Dijk H, Jansen H, Castro Cabezas M. Effects of atorvastatin on fasting and postprandial complement component 3 response in familial combined hyperlipidemia. J Lipid Res. 2003; 44:2100-8.

26. Szeplaki G, Varga L, Laki J et al. Elevated complement C3 is associated with early restenosis after eversion carotid endarterectomy. Thromb Haemost. 2006; 96:529-34.

27. Derzsy Z, Prohászka Z, Rigó J Jr, Füst G, Molvarec A. Activation of the complement system in normal pregnancy and preeclampsia. Mol Immunol. 2010; 47:1500-6.

28. Wamba PC, Mi J, Zhao XY et al. Acylation stimulating protein but not complement C3 associates with metabolic syndrome components in Chinese children and adolescents. Eur J Endocrinol. 2008; 159:781-90.

29. Bhakdi S, Torzewski M, Klouche M, Hemmes M. Complement and atherogenesis: binding of CRP to degraded, nonoxidized LDL enhances complement activation. Arterioscler Thromb Vasc Biol. 1999; 19:2348-54.

30. Bhakdi S, Torzewski M, Paprotka K et al. Possible protective role for C-reactive protein in atherogenesis: complement activation by modified lipoproteins halts before detrimental terminal sequence. Circulation. 2004; 109:1870-6.

31. Seifert PS, Hugo F, Tranum-Jensen J, Zahringer U, Muhly M, Bhakdi S. Isolation and characterization of a complement-activating lipid extracted from human atherosclerotic lesions. J Exp Med. 1990; 172:547-57.

32. Hollander W, Colombo MA, Kirkpatrick B, Paddock J. Soluble proteins in the human atherosclerotic plaque. With spectral reference to immunoglobulins, C3-complement component, alpha 1-antitrypsin and alpha 2-macroglobulin. Atherosclerosis. 1979; 34:391-405. 
33. Niculescu F, Rus HG, Vlaicu R. Immunohistochemical localization of C5b-9, S-protein, C3d and apolipoprotein B in human arterial tissues with atherosclerosis. Atherosclerosis. 1987; 65:111.

34. Yasojima K, McGeer PL, Schwab C, McGeer EG. Generation of C-reactive protein and complement components in atherosclerotic plaques. Am J Pathol. 2001; 158:1039-51.

35. Saito E, Fujioka T, Kanno $\mathrm{H}$ et al. Complement receptors in atherosclerotic lesions. Artery. 1992;19:47-62.

36. Schepers A, de Vries MR, van Leuven CJ et al. Circulation. 2006 Dec 19;114(25):2831-8. Inhibition of complement component C3 reduces vein graft atherosclerosis in apolipoprotein E3-Leiden transgenic mice. Circulation 2006; 114:2831-8.

37. Kostner KM, Fahti RB, Case C, Hobson P, Tate J, Marwick TH. Inflammation, complement activation and endothelial function in stable and unstable coronary artery disease. Clin Chim Acta. 2006; 365:129-34.

38. Onat A, Hergenç G, Can G, Kaya Z, Yüksel H. Serum complement C3: a determinant of cardiometabolic risk, additive to the metabolic syndrome, in middle-aged population. Metabolism 2010; 59:628-34. 


\section{Chapter 5}

The association between the metabolic syndrome and alanine amino transferase is mediated by insulin resistance via related metabolic intermediates (the CODAM study) 


\begin{abstract}
Objective: The metabolic syndrome is associated with non-alcoholic fatty liver disease (NAFLD) as well as with insulin resistance, inflammatory adipokines, endothelial dysfunction and higher plasma levels of non-esterified fatty acids (NEFA), all of which may also affect the development of NAFLD. Therefore we investigated to what extent the association between the metabolic syndrome and alanine aminotransferase (ALT, as a surrogate of NAFLD), can be explained by different metabolic intermediates of the metabolic syndrome. Materials and methods: Cross-sectional analyses in 434 subjects from the Cohort on Diabetes and Atherosclerosis Maastricht study (264 men, mean age 59.5 \pm 7.1 years). We used multiple linear regression analyses to investigate the association between the metabolic syndrome and ALT and the mediation role of potential mediators herein. The mediators considered were insulin resistance (HOMA2IR), an inflammatory adipokine score (based on interleukin 6, serum amyloid A, intercellular adhesion molecule, adiponectin and leptin), an endothelial dysfunction score (based on E-selectin, vascular cell adhesion molecule and von Willebrand factor) and plasma levels of NEFA. All analyses were adjusted for age, sex, smoking, alcohol consumption and use of medication. Results: Subjects with the metabolic syndrome $(53.7 \%)$ had significantly higher levels of ALT ( $\beta=0.67$ SD (95\%CI: 0.49;0.85), $\mathrm{p}<0.001)$. Adjustment for insulin resistance attenuated this difference by $77.3 \%$ (to 0.15 SD (-0.04;0.35)). Attenuation by adipose tissue-associated inflammation, endothelial dysfunction and NEFA was more modest (20.7, 13.1 and $9.5 \%$ respectively). Part of the attenuation by NEFA, but not of the other mediators, was additional to that of insulin resistance. Conclusions: Insulin resistance constitutes a key pathophysiological mechanism in the association between the metabolic syndrome and NAFLD (measured as ALT), which may operate through adipose tissue-associated inflammation and endothelial dysfunction and to a lesser extent through NEFA, which may have an independent role in the development of NAFLD in subjects with the metabolic syndrome.
\end{abstract}




\subsection{Introduction}

The increasing prevalence of the metabolic syndrome draws a heavy burden on public health. Non-alcoholic fatty liver disease (NAFLD) is a common liver disorder that affects up to $20-30 \%$ of the population in the developed world $[1,2]$ and is a very prevalent finding in subjects with the metabolic syndrome [3]. Given the frequency of NAFLD in obesity and type 2 diabetes (T2DM), the prevalence of NAFLD in the metabolic syndrome can be estimated to be at least $70-80 \%[4,5]$.

NAFLD has a wide histological spectrum ranging from simple steatosis and nonalcoholic steatohepatitis (NASH) to more progressive forms of the disease such as fibrosis and eventually cirrhosis. Patients with simple steatosis are, among others, characterized by the accumulation of triglycerides in hepatocytes, while in NASH the accumulation of fat is accompanied by hepatic inflammation [1]. The 'gold standard' to diagnose NAFLD is liver biopsy $[1,6]$. However, this is an invasive procedure with risk of post-interventional bleeding and therefore not acceptable without clinical indication. Alternative methods to detect hepatic fat accumulation are used in epidemiological studies including imaging techniques and measurement of circulating markers such as alanine aminotransferase (ALT) [6-8].

Several unfavourable metabolic syndrome-associated processes may affect the development of NAFLD. Insulin resistance is most likely involved in the initiation of hepatic steatosis, but NAFLD may in turn contribute to further progression of insulin resistance [9]. In adipose tissue, loss of insulin sensitivity can lead to increased release of non-esterified fatty acids (NEFA) in the circulation, which may be incorporated into hepatic triglycerides $[10,11]$. In combination with insufficient elimination of triglycerides, probably caused by hepatic insulin resistance, this can contribute to the development of NAFLD. Inflammation of adipose tissue, as commonly seen in the metabolic syndrome [12], may also contribute to the development of NAFLD. This is firstly because adipose tissue-associated inflammation yields insulin resistant adipocytes by activating JNK and IKK $\beta$ pathways [13, 14], and secondly because inflammatory adipokines contribute to systemic low-grade inflammation that may trigger hepatic inflammation and hepatic insulin resistance and thereby predispose to NAFLD [15]. Another metabolic syndrome-associated process that may contribute to the development of NAFLD is (local) endothelial dysfunction. The liver is a highlyperfused organ and disturbance of liver perfusion may disturb the delicate balance between the supply and removal of nutrients and metabolites [16]. Insulin resistance as well as the concomitant increase in NEFA may disturb microvascular function [17] and thereby possibly contribute to the development and/or progression of NAFLD [18]. The aforementioned pathways are not necessarily fully independent; they may also affect one another. 
Taken together, the evidence so far suggests that insulin resistance, inflammatory adipokines, endothelial dysfunction, and NEFA may each explain, at least in part, the association between the metabolic syndrome and NAFLD. To gain more insight into these issues, we have determined the association between the metabolic syndrome and plasma ALT (as a systemic marker of NAFLD) and investigated the extent to which this association could be explained by insulin resistance, inflammatory adipokines, endothelial dysfunction, and/or NEFA.

\subsection{Materials and methods}

\subsubsection{Subjects and study design}

Cross-sectional analyses were performed on data from the Cohort on Diabetes and Atherosclerosis Maastricht (CODAM) study, which includes 574 subjects with an elevated risk for T2DM and CVD as described in detail elsewhere [19]. In short, all subjects were Caucasian, older than 40 years and met at least one of the following criteria: body mass index above $25 \mathrm{~kg} / \mathrm{m}^{2}$, a positive family history for T2DM, a history of gestational diabetes, use of anti-hypertensive medication, a postprandial glucose above $6.0 \mathrm{mmol} / \mathrm{L}$ and/or glycosuria. The study was approved by the Medical Ethical Committee of the Maastricht University and all subjects gave written informed consent.

Subjects were excluded if they had missing data on one or more of the markers for insulin resistance, inflammatory adipokines, endothelial dysfunction and/or NEFA $(n=44)$. Additionally subjects were excluded if they used insulin therapy $(n=11)$ since in those subjects insulin resistance cannot be estimated by HOMA2IR. Next, those with self-reported liver disease $(n=6)$, or alcohol consumption of more than $20 \mathrm{~g} /$ day (women) or $40 \mathrm{~g} /$ day (men) $(\mathrm{n}=79)$ were excluded, since this is the maximal amount of alcohol consumption that has been accepted in NAFLD [20]. The present study population therefore consisted of a total of 434 individuals.

\subsubsection{Laboratory measurements}

The metabolic syndrome was defined according to the updated definition of the American Heart Association and the National Heart, Lung and Blood Institute, 2005 [21]. According to the results of a standard $75 \mathrm{~g}$ oral glucose tolerance test, 146 subjects had T2DM [22]. Of these T2DM subjects, 55\% were not aware of their diabetes status prior to the screening. Use of medication, smoking behaviour and alcohol consumption were assessed in extensive interview sessions and researchassistant administered questionnaires. Waist circumference was measured at the level midway between the lateral lower rib margin and the spina iliaca anterior superior. Blood pressure was measured twice after 5 minutes of rest with an oscillometric 
precision blood pressure instrument (Maxi stable 3, Speidel \& Keller, currently: Welch Allyn Inc, Skaneateles Falls, New York, USA) on the right arm in the supine position. Subjects were asked to stop their lipid-lowering medication 14 days before the visit and all other medication was stopped the day before the visit ( $>80 \%$ adherence). Blood samples were obtained by venipuncture to determine glucose, cholesterol, triglycerides, interleukin 6 (IL6), serum amyloid A (SAA), soluble intercellular adhesion molecule (sICAM-1) and soluble vascular cell adhesion molecule (sVCAM-1) as described previously [23]. Adiponectin and leptin were measured by ELISA in EDTA plasma (BioVendor Laboratory Medicine, Brno, Czech Republic). sE-selectin was measured in EDTA plasma with a CD62-Elipair ELISA (Diaclone, Tepnel, Besancon Cedex, France). Von Willebrand factor (vWF) was measured in citrate plasma as described previously [24]. NEFA was measured in EDTA plasma using an enzymatic colorimetric method (NEFA-C, Wako Chemicals GmbH, Neuss, Germany) and ALT was measured in EDTA plasma as described before and used as a measure of hepatic fat accumulation [7]. Insulin was measured in EDTA plasma using a 2-sided radioimmunoassay (Medgenix Diagnostics, Brussels, Belgium). Insulin resistance was derived from the homeostasis model assessment (HOMA2IR) and was computed using software downloaded at http://www.dtu.ox.ac.uk.

\subsubsection{Statistical analysis}

Variables with a skewed distribution, i.e. triglycerides, ALT, HOMA2IR, IL6, SAA, sICAM-1, adiponectin, leptin, NEFA, sE-selectin, sVCAM-1 and vWF were logtransformed prior to further analyses. Differences between the subjects with and without the metabolic syndrome were assessed by means of Student's t tests for continuous variables and by Chi-square tests for categorical variables.

Two composite measures (average z-scores) were calculated to reduce the problem of multiple testing. Firstly, an inflammatory adipokines score, which was the average of the z-scores of IL6, SAA, sICAM-1, leptin and the z-score of adiponectin multiplied by -1 . These five markers have each been reported to be secreted by adipose tissue in relevant amounts and have inflammatory (IL6, SAA, sICAM-1, leptin) or antiinflammatory (adiponectin) properties [25-28]. We therefore considered these markers good representatives of adipose tissue-associated inflammation as often seen in the metabolic syndrome. And secondly, an endothelial dysfunction score as the average of the $z$-scores of sE-selectin, sVCAM- 1 and $\mathrm{vWF}$. In order to enable direct comparison of the strengths of all associations, z-scores were also calculated for HOMA2IR, NEFA and ALT.

We used linear regression analyses to investigate the associations, first, between the metabolic syndrome (main independent variable) and ALT (main dependent variable); second, between the metabolic syndrome and the potential mediators, i.e. insulin resistance, inflammatory adipokines, endothelial dysfunction or NEFA; and third, 
between the potential mediators and ALT. Finally we examined the extent to which the association between the metabolic syndrome and ALT was explained, i.e. potentially mediated, by the potential mediators considered. This was done by quantifying, in percentage, the attenuations in the magnitude of the linear regression coefficient reflecting the association between the metabolic syndrome and ALT after adjustment for those mediators. Since there was no interaction between sex and the metabolic syndrome in the association with ALT, analyses were conducted in the whole population. All analyses were adjusted for age, sex, smoking, alcohol consumption, and the use of lipid-lowering and antihypertensive medication.

All statistical analyses were performed using the SPSS package version 15.0 (SPSS, Chicago, IL, USA) and statistical significance was set at $\mathrm{p}<0.05$.

\subsection{Results}

The basic characteristics of the study population are shown in Table 5.1. The prevalence of the metabolic syndrome was $53.7 \%$. Subjects with the metabolic syndrome had higher levels of plasma of ALT, NEFA and inflammatory adipokines, had worse endothelial function and were more insulin resistant compared with those without the metabolic syndrome. 
Table 5.1: Characteristics of the CODAM cohort according to the absence or presence of the metabolic syndrome (MetS)

\begin{tabular}{|c|c|c|c|}
\hline & $\begin{array}{l}\text { Without MetS } \\
\qquad(n=201)\end{array}$ & $\begin{array}{l}\text { With MetS } \\
(\mathrm{n}=233)\end{array}$ & p-value \\
\hline Men/Women (n) & $119 / 82$ & $145 / 88$ & 0.519 \\
\hline Age (years) & $58.7 \pm 7.5$ & $60.2 \pm 6.7$ & 0.034 \\
\hline Waist (cm) & $93.0 \pm 9.8$ & $104.5 \pm 10.8$ & $<0.001$ \\
\hline HDL cholesterol (mmol/L) & $1.36 \pm 0.31$ & $1.01 \pm 0.25$ & $<0.001$ \\
\hline Triglycerides (mmol/L) & $1.1(0.8-1.4)$ & $1.8(1.4-2.2)$ & $<0.001$ \\
\hline Systolic blood pressure (mmHg) & $134 \pm 19$ & $145 \pm 18$ & $<0.001$ \\
\hline Diastolic blood pressure (mmHg) & $79 \pm 9$ & $84 \pm 9$ & $<0.001$ \\
\hline Fasting plasma glucose $(\mathrm{mmol} / \mathrm{L})$ & $5.4 \pm 0.6$ & $6.5 \pm 1.6$ & $<0.001$ \\
\hline Alanine aminotransferase (mmol/L) & $18.5(14.6-24.2)$ & $23.8(19.4-31.4)$ & $<0.001$ \\
\hline Use of anti-hypertensive medication (\%) & 23.9 & 50.2 & $<0.001$ \\
\hline Use of lipid-lowering medication (\%) & 12.9 & 23.2 & 0.006 \\
\hline Type 2 diabetes (\%) & 6.0 & 36.5 & $<0.001$ \\
\hline HOMA2IR & $0.89(0.73-1.09)$ & $1.5(1.2-2.2)$ & $<0.001$ \\
\hline Soluble E-selectin (ng/mL) & $68(48-89)$ & $91(68-120)$ & $<0.001$ \\
\hline Soluble vascular cell adhesion molecule $1(\mathrm{ng} / \mathrm{mL})$ & $447(386-512)$ & $474(387-566)$ & 0.021 \\
\hline Von Willebrand factor ( $\%$ of gold standard) & $116(92-159)$ & $122(93-163)$ & 0.596 \\
\hline Endothelial dysfunction score & $-0.17 \pm 0.58$ & $0.14 \pm 0.62$ & $<0.001$ \\
\hline Interleukin $6(\mathrm{pg} / \mathrm{mL})$ & $1.1(0.8-1.7)$ & $1.6(1.1-2.4)$ & $<0.001$ \\
\hline Serum amyloid $\mathrm{A}(\mu \mathrm{g} / \mathrm{mL})$ & $6.3(4.0-15.0)$ & $8.0(4.6-14.6)$ & 0.320 \\
\hline Soluble intercellular adhesion molecule $1(\mathrm{ng} / \mathrm{mL})$ & $315(272-367)$ & $356(307-411)$ & $<0.001$ \\
\hline Adiponectin $(\mu \mathrm{g} / \mathrm{mL})$ & $8.3(6.4-11.5)$ & $6.4(4.3-8.2)$ & $<0.001$ \\
\hline Leptin (ng/mL) & $7.2(3.7-16.8)$ & $13.0(8.1-24.4)$ & $<0.001$ \\
\hline Inflammatory adipokine score & $-0.24 \pm 0.52$ & $0.21 \pm 0.51$ & $<0.001$ \\
\hline Non-esterified fatty acids (mmol/L) & $0.47(0.36-0.57)$ & $0.52(0.43-0.61)$ & $<0.001$ \\
\hline
\end{tabular}

Data are expressed as $\mathrm{n}$ or frequency $(\%)$, mean \pm standard deviation or median (inter-quartile range);

HDL: high-density lipoprotein, HOMA2IR: homeostasis model assessment of insulin resistance

After adjustment for age, sex, smoking, alcohol consumption, and use of lipidlowering and antihypertensive medication, subjects with the metabolic syndrome had 
significantly higher levels of ALT than those without (linear regression coefficient $\beta$ $(95 \% \mathrm{CI})=0.67 \mathrm{SD}(0.49 ; 0.85), \mathrm{p}<0.001)$. The metabolic syndrome was also positively associated with insulin resistance, inflammatory adipokines, endothelial dysfunction, and NEFA (Table 5.2) and all these four potential mediators were also associated with ALT (Table 5.3, model 1), even after additional adjustment for the metabolic syndrome (Table 5.3, model 2).

Table 5.2: Associations of the metabolic syndrome with insulin resistance, endothelial dysfunction, inflammatory adipokines and non-esterified fatty acids

\begin{tabular}{lcc}
\hline & \multicolumn{2}{c}{ Independent variable: metabolic syndrome } \\
\cline { 2 - 3 } Dependent variables & $\beta(95 \% \mathrm{CI})$ & p-value \\
\hline HOMA2IR & $1.094(0.932 ; 1.255)$ & $<0.001$ \\
Inflammatory adipokines & $0.391(0.291 ; 0.490)$ & $<0.001$ \\
Endothelial dysfunction & $0.212(0.099 ; 0.324)$ & $<0.001$ \\
Non-esterified fatty acids & $0.363(0.169 ; 0.558)$ & $<0.001$ \\
\hline
\end{tabular}

All analyses were adjusted for age, sex, smoking, alcohol consumption, and use of lipid-lowering and antihypertensive medication;

$\beta$ indicates difference in dependent variable (expressed in SD) between subjects with vs. without the metabolic syndrome; HOMA2IR: homeostasis model assessment of insulin resistance

Table 5.3: Associations of insulin resistance, endothelial dysfunction, inflammatory adipokines and nonesterified fatty acids with ALT

\begin{tabular}{|c|c|c|c|c|}
\hline \multirow[b]{3}{*}{ Independent variables } & \multicolumn{4}{|c|}{ Dependent variable: $\log$-ALT } \\
\hline & \multicolumn{2}{|c|}{$\begin{array}{l}\text { Model 1: adjusted for age, sex, } \\
\text { smoking, alcohol consumption, } \\
\text { medication }\end{array}$} & \multicolumn{2}{|c|}{$\begin{array}{l}\text { Model 2: adjusted for age, sex, } \\
\text { smoking, alcohol consumption, } \\
\text { medication, metabolic syndrome }\end{array}$} \\
\hline & $\beta(95 \% \mathrm{CI})$ & p-value & $\beta(95 \% \mathrm{CI})$ & p-value \\
\hline HOMA2IR & $0.514(0.432 ; 0.595)$ & $<0.001$ & $0.472(0.375 ; 0.569)$ & $<0.001$ \\
\hline Inflammatory adipokines & $0.523(0.358 ; 0.688)$ & $<0.001$ & $0.355(0.184 ; 0.525)$ & $<0.001$ \\
\hline Endothelial dysfunction & $0.499(0.347 ; 0.652)$ & $<0.001$ & $0.413(0.265 ; 0.561)$ & $<0.001$ \\
\hline Non-esterified fatty acids & $0.229(0.139 ; 0.319)$ & $<0.001$ & $0.178(0.092 ; 0.265)$ & $<0.001$ \\
\hline
\end{tabular}

$\beta$ indicates difference in $\log$-ALT (expressed in SD (one SD equals $0.16 \log (\mathrm{mmol} / \mathrm{L})$ )) per one SD increase in each independent variable; HOMA2IR: homeostasis model assessment of insulin resistance 
The differences in ALT levels between subjects with and without the metabolic syndrome were attenuated by adjustment for insulin resistance $(77.3 \%$; Table 5.4 , model 2), and to a lesser extent by adjustment for inflammatory adipokines $(20.7 \%$, model 3a), endothelial dysfunction $(13.1 \%$, model $4 \mathrm{a})$, or NEFA $(9.5 \%$, model $5 \mathrm{a})$. Of note, mediation by endothelial dysfunction $(1.8 \%$, model $3 \mathrm{~b})$ and inflammatory adipokines $(-0.4 \%$, model $4 \mathrm{~b})$ did not add to that by insulin resistance. This was different for NEFA, which by itself mediated $9.5 \%$ of the association and added $4.4 \%$ to the mediation of insulin resistance (model 5b).

When the metabolic syndrome, insulin resistance, inflammatory adipokines, endothelial dysfunction, and NEFA were added in a full model including also age, sex, smoking, alcohol consumption, and use of lipid-lowering and antihypertensive medication, insulin resistance $(0.41(0.30 ; 0.51) \mathrm{p}<0.001)$, endothelial dysfunction (0.21 (0.06;0.036) $\mathrm{p}=0.005)$ and NEFA $(0.13(0.05 ; 0.21) \mathrm{p}=0.002)$, but not the metabolic syndrome or inflammatory adipokines, were significantly associated with plasma ALT. 
Table 5.4: Associations of the metabolic syndrome with alanine aminotransferase and the mediation by insulin resistance, endothelial dysfunction, inflammatory adipokines and non-esterified fatty acids

\begin{tabular}{|c|c|c|c|c|c|}
\hline \multirow[b]{2}{*}{ Model } & \multirow[b]{2}{*}{ Adjustments } & \multicolumn{4}{|c|}{ Independent variable: Metabolic syndrome } \\
\hline & & $\beta(95 \% \mathrm{CI})$ & p-value & mediation* $(\%)$ & $\begin{array}{c}\text { additional } \\
\text { mediation }^{\#}(\%)\end{array}$ \\
\hline 1 & $\begin{array}{l}\text { Age, sex, smoking, alcohol } \\
\text { consumption, medication }\end{array}$ & $\begin{array}{c}0.671 \\
(0.490 ; 0.851)\end{array}$ & $<0.001$ & & \\
\hline 2 & $\begin{array}{l}\text { Model } 1+ \\
\text { HOMA2IR }\end{array}$ & $\begin{array}{c}0.152 \\
(-0.043 ; 0.347)\end{array}$ & 0.127 & 77.3 & \\
\hline $3 a$ & $\begin{array}{l}\text { Model } 1+ \\
\text { inflammatory adipokines }\end{array}$ & $\begin{array}{c}0.532 \\
(0.343 ; 0.721)\end{array}$ & $<0.001$ & 20.7 & \\
\hline $3 \mathrm{~b}$ & $\begin{array}{l}\text { Model } 1+\text { HOMA2IR + } \\
\text { inflammatory adipokines }\end{array}$ & $\begin{array}{c}0.140 \\
(-0.056 ; 0.337)\end{array}$ & 0.161 & 79.1 & 1.8 \\
\hline $4 a$ & $\begin{array}{l}\text { Model } 1+ \\
\text { endothelial dysfunction }\end{array}$ & $\begin{array}{c}0.583 \\
(0.406 ; 0.760)\end{array}$ & $<0.001$ & 13.1 & \\
\hline $4 \mathrm{~b}$ & $\begin{array}{l}\text { Model } 1+\text { HOMA2IR + } \\
\text { endothelial dysfunction }\end{array}$ & $\begin{array}{c}0.155 \\
(-0.038 ; 0.348)\end{array}$ & 0.115 & 76.9 & -0.4 \\
\hline $5 \mathrm{a}$ & $\begin{array}{l}\text { Model } 1+ \\
\text { nonesterified fatty acids }\end{array}$ & $\begin{array}{c}0.607 \\
(0.427 ; 0.786)\end{array}$ & $<0.001$ & 9.5 & \\
\hline $5 b$ & $\begin{array}{l}\text { Model } 1+\text { HOMA2IR + } \\
\text { nonesterified fatty acids }\end{array}$ & $\begin{array}{c}0.123 \\
(-0.070 ; 0.317)\end{array}$ & 0.211 & 81.7 & 4.4 \\
\hline
\end{tabular}

$\beta$ indicates difference in alanine aminotransferase (expressed in SD) between subjects with vs. without the metabolic syndrome; HOMA2IR: Homeostasis model assessment of insulin resistance;

* indicates change in the magnitude of the regression coefficient $(\beta)$ as compared to Model 1 (expressed in \%);

\# indicates the difference in the \% attenuation by: HOMA2IR and inflammatory adipokines (model 3b), by HOMA2IR and endothelial dysfunction (model 4b) or by HOMA2IR and nonesterified fatty acids (model 5b) in comparison to the $\%$ mediation by HOMA2IR only (model 2).

\subsubsection{Additional analyses}

In the above-described analyses we used an average z-score for inflammatory adipokines assuming that these markers represent adipose tissue-associated inflammation as often seen in the metabolic syndrome. To exclude that our current results merely represent general low-grade inflammation, the analyses were repeated with C-reactive protein (CRP) as potential mediator. CRP did not essentially mediate the association of the metabolic syndrome with ALT; mediation by CRP was $4.2 \%$, as compared to the $20.7 \%$ that was observed for the inflammatory adipokine score. 
It has been reported that plasma ALT levels may underestimate liver fat content in T2DM patients [29]. The analyses reported above were therefore repeated without subjects with T2DM and the results of these analyses did not materially differ from what was reported above. The analyses were also repeated with a more stringent cutoff value for alcohol consumption i.e. by excluding all subjects who consume $>20 \mathrm{~g}$ alcohol daily, since this is the proposed threshold to distinguish between nonalcoholic and alcoholic FLD [20]. Again, the results of these analyses did not materially differ from what was reported above (data not shown).

\subsection{Discussion}

In this study we showed that among the several metabolic syndrome-associated processes that were investigated, insulin resistance was the strongest mediator of the association between the metabolic syndrome and ALT while inflammatory adipokines, endothelial dysfunction, and NEFA also explained a part of the association between the metabolic syndrome and ALT, but to a lesser extent. Inflammatory adipokines and endothelial dysfunction, however, did not add to the mediation by insulin resistance while approximately half of the mediation by NEFA was additional to that by insulin resistance.

Because of well-known mutual relations between the metabolic syndrome, insulin resistance and steatosis [30], the strong mediation by insulin resistance was largely anticipated. A more interesting finding is that although inflammatory adipokines were by themselves moderate mediators of the association between the metabolic syndrome and ALT, this effect was abolished when insulin resistance was also included in the model. This suggests that these processes, at least partly, represent the same mechanism(s). The overlap between mediation by inflammatory adipokines and insulin resistance may be explained by the fact that compromised insulin signalling and/or hyperinsulinaemia coincide with an altered production of these adipokines. Data in the literature show that the production of leptin by human adipocytes is regulated by hyperinsulinaemia and/or insulin signalling [31] as is the production of adiponectin [32,33] and IL6 [34, 35]. Insulin signalling affects ICAM expression in leucocytes [36] and high insulin concentrations increase the expression of ICAM on endothelial cells [37]. Some of these adipokines may subsequently or perhaps concomitantly worsen (adipose) insulin resistance via activation of inflammatory pathways [38]. Thus, our data suggest that inflammatory adipokines may be responsible for a part of the mediating effect of insulin resistance. Our finding that it is inflammation of adipose tissue rather than general inflammation accentuates the importance of adipose tissue dysfunction in the association of the metabolic syndrome with ALT, and supports the use of the inflammatory adipokine score. 
The observed (modest) mediation by endothelial dysfunction in the association between the metabolic syndrome and ALT also disappeared after concomitant adjustments for insulin resistance. This may be explained by direct effects of hyperinsulinaemia and/or insulin signalling on endothelial dysfunction [39] and additionally by effects of inflammatory adipokines on endothelial dysfunction [40]. Since we have only systemic markers of endothelial dysfunction available and because of the cross-sectional design of our study, currently we can not distinguish between the possibility that local insulin resistance in the liver is causal in the generation of fatty liver and the alternative explanation that an overall status of endothelial dysfunction is merely a reflection of the insulin resistant state. Although this was not the primary aim of our analyses, we did observe that endothelial dysfunction was associated with ALT, independently of insulin resistance and the other potential mediators considered, suggesting that it can also be an operative pathophysiological mechanism linking risk factors other than the metabolic syndrome to ALT. Taken together, although these data must be interpreted with caution, our findings support the possibility that insulin resistance-associated adipose tissue inflammation and hepatic microvascular dysfunction might actually contribute to the development and/or progression of ALT/NAFLD in the metabolic syndrome.

Increased plasma NEFA concentrations are a hallmark of the metabolic syndrome and it is generally accepted that plasma NEFA contribute to hepatic triglyceride accumulation and the development of NAFLD [10]. We had hypothesized that mediation of the association between the metabolic syndrome and ALT by NEFA would be similar to that by inflammatory adipokines because both adipose insulin resistance and adipose inflammation are known to induce fatty acid release from adipose tissue. However, NEFA mediated the association more modestly than inflammatory adipokines $(9.5 \%$ vs. $20.7 \%)$, but despite this and in contrast to the latter, mediation by NEFA was not completely abolished in the presence of concomitant mediation by insulin resistance. Our data suggest that half of the mediation attributable to NEFA may occur via pathways included in the process of adipose tissue insulin resistance and inflammation, while the remaining half most likely occurs via an insulin resistance-independent mechanism. Data in the literature report that in upper-body obese women, upper-body subcutaneous, not visceral, fat was main source of plasma NEFA [41] and systemic NEFA have been reported to be a major source of the extrahepatic fatty acids that accumulate in NAFLD [10]. These NEFA may contribute to hepatic steatosis partly via increased insulin resistance and inflammation as described above and partly via direct uptake into hepatocytes. These latter associations of dietary fatty acids and direct uptake of subcutaneous adipose tissue-derived NEFA may also be reflected by the significant independent contribution of NEFA to plasma ALT levels in the full model (including all potential mediators). 
In this study, we particularly focused on potential mediators that were not included in the metabolic syndrome since these may provide insight in the metabolic pathways that are intermediate between the metabolic syndrome and ALT levels. We did not investigate the extent to which each of the individual components of the metabolic syndrome contributed to the relation between the metabolic syndrome and ALT levels. Some prospective studies showed that plasma concentrations of ALT could predict the metabolic syndrome $[42,43]$. The latter also showed that a substantial part of this association was explained by insulin resistance, which is in line with our current findings.

A limitation of our study may be the use of ALT levels as a measure of hepatic fat accumulation. However, even though ALT levels are not the ideal measure of hepatic fat accumulation the associations of ALT with the metabolic syndrome and with insulin resistance, inflammation, NEFA and endothelial dysfunction, reported herein are in agreement with those reported by others when other measures for hepatic fat accumulation were used $[9,16,44,45]$. Other limitations of our study include its cross-sectional design, which prohibits conclusions about causality, and the fact that we studied middle-aged Caucasians, which excludes extrapolation of our results to other age categories or other ethnicities.

In conclusion, we showed that insulin resistance mediates up to $75-80 \%$ of the association of the metabolic syndrome with ALT. Our findings additionally suggest that insulin resistance, and its associated adipose tissue inflammation and endothelial dysfunction may contribute to the progression of fatty liver disease (measured as ALT), via a common pathophysiological mechanism while the effect of NEFA may be partly independent of insulin resistance. Treatment of insulin resistance, which may concomitantly ameliorate endothelial dysfunction and the amount of circulating inflammatory cytokines, thus remains a main target for interventions aiming at the prevention of NAFLD in these individuals. Circulating levels of NEFA may also constitute an additional treatment target.

\subsection{References}

1 de Alwis NM, Day CP. Non-alcoholic fatty liver disease: the mist gradually clears. J Hepatol 2008;48 Suppl 1(S104-112.

2 Younossi ZM. Review article: current management of non-alcoholic fatty liver disease and nonalcoholic steatohepatitis. Aliment Pharmacol Ther 2008;28(1):2-12.

3 Liangpunsakul S, Chalasani N. Unexplained elevations in alanine aminotransferase in individuals with the metabolic syndrome: results from the third National Health and Nutrition Survey (NHANES III). Am J Med Sci 2005;329(3):111-116.

4 Bellentani S, Saccoccio G, Masutti F, et al. Prevalence of and Risk Factors for Hepatic Steatosis in Northern Italy. Annals of Internal Medicine 2000;132(2):112-117. 
Jeong SK, Kim YK, Park JW, et al. Impact of visceral fat on the metabolic syndrome and nonalcoholic fatty liver disease. J Korean Med Sci 2008;23(5):789-795.

6 Brunt EM, Janney CG, Di Bisceglie AM, et al. Nonalcoholic steatohepatitis: a proposal for grading and staging the histological lesions. Am J Gastroenterol 1999;94(9):2467-2474.

7 Brouwers MC, Cantor RM, Kono N, et al. Heritability and genetic loci of fatty liver in familial combined hyperlipidemia. J Lipid Res 2006;47(12):2799-2807.

8 Siegelman ES, Rosen MA. Imaging of hepatic steatosis. Semin Liver Dis 2001;21(1):71-80.

9 Lonardo A, Lombardini S, Ricchi M, et al. Review article: hepatic steatosis and insulin resistance. Aliment Pharmacol Ther 2005;22 Suppl 2(64-70.

10 Donnelly KL, Smith CI, Schwarzenberg SJ, et al. Sources of fatty acids stored in liver and secreted via lipoproteins in patients with nonalcoholic fatty liver disease. J Clin Invest 2005;115(5):1343-1351.

11 Puri P, Baillie RA, Wiest MM, et al. A lipidomic analysis of nonalcoholic fatty liver disease. Hepatology 2007;46(4):1081-1090.

12 Wellen KE, Hotamisligil GS. Inflammation, stress, and diabetes. J Clin Invest 2005;115(5):1111-1119.

13 Hirosumi J, Tuncman G, Chang L, et al. A central role for JNK in obesity and insulin resistance. Nature 2002;420(6913):333-336.

14 Werner ED, Lee J, Hansen L, et al. Insulin resistance due to phosphorylation of insulin receptor substrate-1 at serine 302. J Biol Chem 2004;279(34):35298-35305.

15 Shoelson SE, Herrero L, Naaz A. Obesity, inflammation, and insulin resistance. Gastroenterology 2007;132(6):2169-2180.

16 Schindhelm RK, Diamant M, Bakker SJ, et al. Liver alanine aminotransferase, insulin resistance and endothelial dysfunction in normotriglyceridaemic subjects with type 2 diabetes mellitus. Eur J Clin Invest 2005;35(6):369-374.

17 Potenza MA, Addabbo F, Montagnani M. Vascular Actions of Insulin with Implications for Endothelial Dysfunction. Am J Physiol Endocrinol Metab 2009;

18 Brock RW, Dorman RB. Obesity, insulin resistance and hepatic perfusion. Microcirculation 2007;14(4-5):339-347.

19 Kruijshoop M, Feskens EJ, Blaak EE, et al. Validation of capillary glucose measurements to detect glucose intolerance or type 2 diabetes mellitus in the general population. Clin Chim Acta 2004;341(1-2):33-40.

20 Neuschwander-Tetri BA, Caldwell SH. Nonalcoholic steatohepatitis: Summary of an AASLD Single Topic Conference. Hepatology 2003;37(5):1202-1219.

21 Grundy SM, Cleeman JI, Daniels SR, et al. Diagnosis and management of the metabolic syndrome: an American Heart Association/National Heart, Lung, and Blood Institute Scientific Statement. Circulation 2005;112(17):2735-2752.

22 WHO. Definition, diagnosis and classification of diabetes mellitus. Report of a WHO Consultation. Part 1: Diagnosis and Classification of Diabetes Mellitus. Geneva, Switzerland: WHO/NCD/NCS/992. 1999; 
23 Jacobs M, van Greevenbroek MM, van der Kallen CJ, et al. Low-grade inflammation can partly explain the association between the metabolic syndrome and either coronary artery disease or severity of peripheral arterial disease: the CODAM study. Eur J Clin Invest 2009;39(6):437-444. Stam F, van Guldener C, Schalkwijk CG, et al. Impaired renal function is associated with markers of endothelial dysfunction and increased inflammatory activity. Nephrol Dial Transplant 2003;18(5):892-898. Brake DK, Smith EO, Mersmann H, et al. ICAM-1 expression in adipose tissue: effects of dietinduced obesity in mice. Am J Physiol Cell Physiol 2006;291(6):C1232-1239. Gustafson B, Hammarstedt A, Andersson CX, et al. Inflamed adipose tissue: a culprit underlying the metabolic syndrome and atherosclerosis. Arterioscler Thromb Vasc Biol 2007;27(11):2276-2283.

Poitou C, Viguerie N, Cancello R, et al. Serum amyloid A: production by human white adipocyte and regulation by obesity and nutrition. Diabetologia 2005;48(3):519-528.

O'Brien KD, Chait A. Serum amyloid A: the "other" inflammatory protein. Curr Atheroscler Rep 2006;8(1):62-68.

29 Kotronen A, Juurinen L, Hakkarainen A, et al. Liver fat is increased in type 2 diabetic patients and underestimated by serum alanine aminotransferase compared with equally obese nondiabetic subjects. Diabetes Care 2008;31(1):165-169.

30 Yki-Jarvinen $\mathrm{H}$, Westerbacka J. The fatty liver and insulin resistance. Curr Mol Med 2005;5(3):287-295.

31 Lee M-J, Fried SK. Integration of hormonal and nutrient signals that regulate leptin synthesis and secretion. Am J Physiol Endocrinol Metab 2009;296(6):E1230-1238.

32 Blümer RME, van Roomen CP, Meijer AJ, et al. Regulation of adiponectin secretion by insulin and amino acids in 3T3-L1 adipocytes. Metabolism 2008;57(12):1655-1662.

33 Sakamoto K, Sakamoto T, Ogawa H. Effects of metabolic risk factors on production of plasminogen activator inhibitor-1 and adiponectin by adipocytes. Circ J 2008;72(5):844-846.

34 Krogh-Madsen R, Plomgaard P, Keller P, et al. Insulin stimulates interleukin-6 and tumor necrosis factor- $\{$ alpha\} gene expression in human subcutaneous adipose tissue. Am J Physiol Endocrinol Metab 2004;286(2):E234-238.

35 LaPensee CR, Hugo ER, Ben-Jonathan N. Insulin Stimulates Interleukin-6 Expression and Release in LS14 Human Adipocytes through Multiple Signaling Pathways. Endocrinology 2008;149(11):5415-5422.

36 Li J, Wu F, Zhang H, et al. Insulin inhibits leukocyte-endothelium adherence via an Akt-NOdependent mechanism in myocardial ischemia/reperfusion. Journal of Molecular and Cellular Cardiology 2009;47(4):512-519.

37 Okouchi M, Okayama N, Omi H, et al. Protective actions of gliclazide on high insulinenhanced neutrophil-endothelial cell interactions through inhibition of mitogen activated protein kinase and protein kinase C pathways. Microvascular Research 2004;67(1):1-8. Hotamisligil GS. Inflammation and metabolic disorders. Nature 2006;444(7121):860-867. 
39 de Jongh RT, Serné EH, Ijzerman RG, et al. Impaired local microvascular vasodilatory effects of insulin and reduced skin microvascular vasomotion in obese women. Microvascular Research 2008;75(2):256-262.

40 Ritchie SA, Ewart M-A, Perry CG, et al. The role of insulin and the adipocytokines in regulation of vascular endothelial function. Clin Sci 2004;107(6):519-532.

41 Martin ML, Jensen MD. Effects of body fat distribution on regional lipolysis in obesity. The Journal of Clinical Investigation 1991;88(2):609-613.

42 Goessling W, Massaro JM, Vasan RS, et al. Aminotransferase levels and 20-year risk of metabolic syndrome, diabetes, and cardiovascular disease. Gastroenterology 2008;135(6):19351944, 1944 e1931.

43 Olynyk JK, Knuiman MW, Divitini ML, et al. Serum alanine aminotransferase, metabolic syndrome, and cardiovascular disease in an Australian population. Am J Gastroenterol 2009;104(7):1715-1722.

44 Lemoine M, Ratziu V, Kim M, et al. Serum adipokine levels predictive of liver injury in nonalcoholic fatty liver disease. Liver Int 2009;

45 Villanova N, Moscatiello S, Ramilli S, et al. Endothelial dysfunction and cardiovascular risk profile in nonalcoholic fatty liver disease. Hepatology 2005;42(2):473-480. 


\section{Chapter 6}

\section{The cross-sectional association between insulin resistance and circulation complement $\mathrm{C} 3$ is partly explained by plasma alanine aminotransferase, independent of central obesity and general inflammation (the CODAM study)}




\begin{abstract}
Background: Complement C3, a central component of the innate immune system is increased in subjects with obesity and type 2 diabetes and is a novel risk factor for cardiovascular disease. We hypothesized that the strong association between insulin resistance and circulating amounts of C3 may be related to hepatic fat accumulation -independent of central obesity itself and of a general low-grade inflammatory response. Research question: To what extent is the association between insulin resistance and C3 explained by plasma levels of alanine aminotransferase (ALT) as a surrogate of hepatic fat accumulation. Methods: Cross-sectional analyses conducted in the Cohort on Diabetes and Atherosclerosis Maastricht (CODAM) study. Analyses were restricted to subjects with none-to-moderate alcohol consumption ( $n=453,61.4 \%$ men). Multiple linear regression analyses were used to investigate the association between HOMA2IR (main determinant) and circulating complement C3 (main outcome), and the mediating role of ALT herein. All analyses were adjusted for age, sex, presence of T2DM or heart disease, use of medication, smoking, alcohol consumption, waist circumference and inflammation. Results: Insulin resistance (estimated as HOMA2IR) was strongly associated with circulating C3 (standardized regression coefficient $\beta 0.40$ (95\% CI: $0.30 ; 0.49))$ and also with ALT $(\beta 0.44(0.34 ; 0.54))$, both adjusted for the above-mentioned covariates. The association between HOMA2IR and C3 was attenuated after further adjustment for ALT ( $\beta$ decreased to $0.34(0.24 ; 0.44)$ ). Concluding remarks: Plasma ALT can explain $14.2 \%$ of the strong association between insulin resistance and circulating concentrations of complement C3, independent of central obesity and general inflammation.
\end{abstract}




\subsection{Introduction}

Complement C3 is an emerging risk factor for cardiometabolic diseases. Positive associations between circulating concentrations C3 and cardiovascular disease have been reported to be independent of traditional risk factors such as smoking, hypertension, cholesterol and diabetes [1-4], although data from a large prospective study suggested this association may actually not be independent of such factors [5]. Complement C3 is substantially increased in the circulation of subjects with the metabolic syndrome [6], is strongly associated with insulin resistance [7], and is a risk factor for development of hypertension and type 2 diabetes mellitus (T2DM) [8,9]. The role of complement C3 with respect to risk of disorders such as, the metabolic syndrome, T2DM and cardiovascular disease has been receiving considerable attention but the metabolic mechanisms that may underlie the increase in circulating concentrations of complement $\mathrm{C} 3$ in these related metabolic disorders are less wellstudied.

Several common aspects of obesity-related metabolic disease may contribute to the increased amounts of circulating C3. Studies on the regulation of C3 production have largely focused on inflammatory and immune processes since complement $\mathrm{C} 3$ is an important component of the innate immune system and C3 production is indeed stimulated by cytokines [10-12]. The association between insulin resistance and C3 may thus be explained as the result of a common antecessor since low-grade inflammation as seen in obesity-related metabolic disorders can induce both insulin resistance and C3 production. Alternatively, this association may, at least in part, be independent of low-grade inflammation since the association between insulin resistance and C3 was shown to be independent of C-reactive protein (CRP) [7]. In this line, some data, although limited, have suggested a direct effect of insulin on complement $\mathrm{C} 3$ production [13].

The larger part of circulating C3 is most likely produced by the liver [14] and Sreekumar et al showed that C3 gene expression is increased in non-alcoholic steatohepatitis (NASH), which is included in the non-alcoholic fatty liver disease (NAFLD) spectrum [15]. Of note, obesity-related metabolic disorders have a very high prevalence of NAFLD, i.e. up to $80 \%[16,17]$ which underlines the potential regulatory role for hepatic fat in the increased levels of circulating C3 in these disorders. In addition, extra hepatic sources such as vascular endothelium $[12,16]$ and adipose tissue $[18,19]$ may also contribute to plasma C3. With respect to the latter, weight loss is associated with a reduction in circulating $\mathrm{C} 3$ concentration, independent of e.g. triglycerides and glucose [20] while C3 by itself may also comprise a risk factor for incurring weight gain [21]. 
Taken together, the evidence suggests that insulin resistance is a major determinant of circulating complement C3. However, the mechanisms through which insulin resistance may affect $\mathrm{C} 3$ levels have not been thoroughly studied. We have therefore investigated to what extent the association between insulin resistance and plasma complement C3 is explained by hepatic fat accumulation (as represented by plasma ALT levels), independent of central obesity and general low-grade inflammation that are generally present in insulin resistance.

\subsection{Methods}

\subsubsection{Study population and design}

The CODAM (Cohort on Diabetes and Atherosclerosis Maastricht) study includes 574 subjects with an elevated risk of T2DM and cardiovascular disease [22]. In short, participants were selected from a large population-based cohort [23]. Inclusion criteria were Caucasian descent and age $>40$ years and, in addition, at least one of the following: body mass index $(\mathrm{BMI})>25 \mathrm{~kg} / \mathrm{m}^{2}$; a positive family history of T2DM; a history of gestational diabetes and/or glucosuria; and use of anti-hypertensive medication. Combinations of these separate inclusion criteria were also allowed. Study participants were extensively characterized with regard to their metabolic, cardiovascular and lifestyle profiles during two visits to the University's research unit. The present study reports on cross-sectional analyses concerning 453 individuals (278 men). Subjects who had missing data on one or more of the markers used in the analyses $(n=26)$, were on insulin therapy $(n=13)$, had self-reported liver disease $(n=7)$ or consumed more than $20 \mathrm{~g} /$ day (women) or $40 \mathrm{~g} /$ day (men) of alcohol i.e. the maximal amount of alcohol consumption that has been accepted in NAFLD [24] $(n=75)$ were excluded from the analyses. The study was approved by the Medical Ethical Committee of the Maastricht University and all subjects gave written informed consent. Reporting of the study conforms to STROBE along with references to STROBE and the broader EQUATOR guidelines [25].

\subsubsection{Laboratory measurements}

Subjects were asked to stop their lipid-lowering medication 14 days before the first visit and all other medication was stopped the day before the visit ( $>80 \%$ adherence). Blood samples were obtained by venipuncture. Serum was allowed to clot at room temperature for $45 \mathrm{~min}$, centrifuged at $3000 \mathrm{rpm}$ for $15 \mathrm{~min}$ and serum aliquots were stored at $-80^{\circ} \mathrm{C}$ until use. Complement C3 was measured in serum by auto analyzer (Hitachi 912) using a Roche kit assay. The interassay variation of the C3 determination was $2.14 \%$ and the average C3 concentration of the reference serum was exactly as reported by the manufacturer (protein Precinorm; C3, $1.36 \mathrm{~g} / \mathrm{L}$ ). Haptoglobin, 
ceruloplasmin and high sensitive CRP were also determined in serum by auto analyzer (Hitachi 912), by using the Tina-Quant Ceruloplasmin assay and by using the CRPLatex assay, respectively (both Roche diagnostics). EDTA blood was collected in precooled tubes on ice, centrifuged at $3000 \mathrm{rpm}$ for $15 \mathrm{~min}$ at $4^{\circ} \mathrm{C}$ and EDTA plasma aliquots were stored at $-80^{\circ} \mathrm{C}$ until use. Plasma insulin concentrations were determined in EDTA plasma with a two-sided immunoradiometric test, using paired monoclonal antibodies (Medgenix Diagnostics). Glucose concentrations were measured in $\mathrm{NaF} / \mathrm{KOx}$ plasma with the hexokinase glucose-6-phosphate dehydrogenase method (HK-G6PD method; ABX Diagnostics Glucose HK 125). Insulin resistance (IR) was estimated according to the homeostasis model assessment (HOMA) using the HOMA2 calculator (www.dtu.ox.ac.uk) [26]. The following variables were also determined in EDTA plasma; HDL-cholesterol (HDL-c) using the HDL-C plus assay, total cholesterol using the CHOD-PAP assay, and triglycerides (TG) using the TG GPO-PAP assay (all Roche diagnostics). High-sensitive interleukin 6 (IL6, R\&D) and serum amyloid A (SAA) and soluble intercellular adhesion molecule (sICAM) (Biosource) were measured using ELISA kits. Alanine aminotransferase (ALT) was measured in EDTA plasma as described before, and used as a measure of hepatic fat accumulation [27].

Waist circumference was measured at the level midway between the lateral lower rib margin and the spina iliaca anterior superior. Height was measured with a stadiometer and weight with a scale with subjects in underwear. BMI was calculated as weight divided by height squared $\left(\mathrm{kg} / \mathrm{m}^{2}\right)$. Blood pressure was measured twice on each visit after 5 minutes of rest with an oscillometric precision blood pressure instrument (Maxi stable 3, Speidel \& Keller) on the right arm with subjects in the supine position. Presence of the metabolic syndrome was determined according to the National Cholesterol Education Program (NCEP) - Adult Treatment Panel (ATP) III definition as modified by the American Heart Association (AHA) / National Heart, Lung and Blood Institute (NHLBI) [28]. Prior cardiovascular disease (CVD) was defined as self-reported myocardial infarction, bypass surgery of the coronary arteries, balloon dilatation, stent placement, stroke or transient ischemic attack (through questionnaires) and/or the presence of signs of myocardial infarction (Minnesota codes 1-1 or 1-2) or ischaemia (Minnesota codes 1-3, 4-1, 4-2, 4-3, 5-1, 5-2, 5-3 or 7-1) on a 12-lead electrocardiogram and (from subjects disease history questionnaire) and/or amputation for non-traumatic reasons and/or ankle-arm-index (AAIx) $<0.9$. For most of the cases ( $>75 \%$ of the subjects with self-reported CVD), the selfreports could be confirmed using available hospital registries. Smoking habits were determined with the help of an extensive questionnaire about current tobacco smoking, smoking in the past, and quitting attempts. Alcohol consumption was assessed by a validated food frequency questionnaire [29]. 


\subsubsection{Statistical analysis}

Variables with a skewed distribution (i.e. insulin, HOMA2IR, ALT, CRP, SAA, IL6 and sICAM) were log-transformed prior to further analyses. Subject characteristics were compared across tertiles of $\mathrm{C} 3$ concentrations by means of ANOVA for continuous variables or by $\mathrm{Chi}^{2}$ tests for categorical variables. We computed a general inflammation score by averaging the z-scores (i.e. (the subject's observed value mean value in the cohort) / cohort SD) of the six markers of low-grade inflammation considered. This composite measure of inflammation was calculated to overcome the problems of multiple testing and misclassification that that could occur when analyzing each marker separately. To be able to directly compare the strengths of all associations, z-scores were also calculated for waist, HOMA2IR, C3 and ALT.

Linear regression analyses were used to determine: firstly, the association between the main determinant, i.e. HOMA2IR, and the main study outcome, i.e. complement C3; secondly, the association between HOMA2IR and ALT; and thirdly, between the ALT and C3. Finally we examined the extent to which the association between HOMA2IR and C3 was explained, i.e. mediated, by ALT in which case magnitude of the linear regression coefficient reflecting the association between HOMA2IR and C3 would be attenuated after further adjustment for ALT. All analyses were adjusted for age, sex, presence of T2DM or prior CVD, use of lipid-lowering, glucose lowering and/or antihypertensive medication, current smoking status (yes/no), alcohol consumption (in $\mathrm{g} /$ day), waist and general inflammation score. All statistical analyses were performed using the SPSS package version 15.0 (SPSS, Chicago, IL, USA) and statistical significance was set at $\mathrm{p}<0.05$.

\subsection{Results}

General characteristics of the study population are shown in Table 6.1. Subjects with higher C3 concentrations were older, more insulin resistant, more obese, had higher circulating concentrations of inflammation-associated factors (i.e. inflammatory cytokines and acute phase response proteins) and more often had CVD, T2DM and the metabolic syndrome. 
Table 6.1: General characteristics of the study population

\begin{tabular}{|c|c|c|c|c|}
\hline & \multicolumn{4}{|c|}{ Tertiles of C3 concentration } \\
\hline & $\begin{array}{l}\text { Lowest } \\
(\mathrm{n}=149)\end{array}$ & $\begin{array}{l}\text { Middle } \\
(\mathrm{n}=155)\end{array}$ & $\begin{array}{l}\text { Highest } \\
(\mathrm{n}=149)\end{array}$ & $\begin{array}{l}\mathrm{P} \text { for } \\
\text { trend }\end{array}$ \\
\hline Age (years) & $58.7 \pm 7.6$ & $60.2 \pm 6.9$ & $59.8 \pm 6.7$ & 0.122 \\
\hline Sex ( $\%$ male $)$ & 65.8 & 61.3 & 57.0 & 0.302 \\
\hline Current smoker & 23.5 & 18.1 & 18.8 & 0.444 \\
\hline BMI $(\mathrm{kg} / \mathrm{m} 2)$ & $26.7 \pm 3.4$ & $28.5 \pm 3.5$ & $30.6 \pm 4.9$ & $<0.001$ \\
\hline Waist (cm) & $93.7 \pm 10.9$ & $99.6 \pm 9.3$ & $104.3 \pm 12.3$ & $<0.001$ \\
\hline Insulin (pM) & $46(38 ; 57)$ & $61(48 ; 83)$ & $85(65 ; 120)$ & $<0.001$ \\
\hline HOMA2IR & $0.88(0.73 ; 1.08)$ & $1.19(0.94 ; 1.62)$ & $1.69(1.25 ; 2.32)$ & $<0.001$ \\
\hline C3 (g/L) & $1.44 \pm 0.13$ & $1.78 \pm 0.10$ & $2.18 \pm 0.21$ & $<0.001$ \\
\hline Alanine aminotransferase $(\mathrm{U} / \mathrm{L})$ & $18.8(14.5 ; 25.0)$ & $22.0(18.0 ; 26.5)$ & $24.6(19.4 ; 32.4)$ & $<0.001$ \\
\hline C-reactive protein $(\mu \mathrm{g} / \mathrm{mL})$ & $1.61(0.96 ; 2.96)$ & $2.76(1.41 ; 4.69)$ & $3.67(2.20 ; 7.14)$ & $<0.001$ \\
\hline Interleukin $6(\mathrm{pg} / \mathrm{mL})$ & $1.18(0.81 ; 1.89)$ & $1.41(0.98 ; 2.11)$ & $1.58(1.03 ; 2.43)$ & 0.002 \\
\hline $\begin{array}{l}\text { Soluble intercellular adhesion molecule } \\
(\mathrm{ng} / \mathrm{mL})\end{array}$ & $323(277 ; 367)$ & $318(283 ; 376)$ & $373(309 ; 420)$ & $<0.001$ \\
\hline Serum amyloid $\mathrm{A}(\mu \mathrm{g} / \mathrm{mL})$ & $6.0(3.6 ; 11.7)$ & $6.5(4.1 ; 13.8)$ & $10.3(5.5 ; 16.9)$ & $<0.001$ \\
\hline Ceruloplasmin $(\mathrm{g} / \mathrm{L})$ & $0.25 \pm 0.04$ & $0.26 \pm 0.04$ & $0.29 \pm 0.07$ & $<0.001$ \\
\hline Haptoglobin $(\mathrm{g} / \mathrm{L})$ & $1.09 \pm 0.45$ & $1.33 \pm 0.46$ & $1.55 \pm 0.55$ & $<0.001$ \\
\hline General inflammation score & $-0.32 \pm 0.57$ & $-0.03 \pm 0.53$ & $0.36 \pm 0.72$ & $<0.001$ \\
\hline Cardiovascular disease $(\%)$ & 20.8 & 25.8 & 40.9 & $<0.001$ \\
\hline Metabolic syndrome (\%) & 26.8 & 57.4 & 81.2 & $<0.001$ \\
\hline Type 2 DM (\%) & 14.8 & 23.2 & 30.9 & 0.004 \\
\hline Use of glucose-lowering medication ( $\%)$ & 8.1 & 12.9 & 15.4 & 0.140 \\
\hline Use of lipid-lowering medication (\%) & 8.7 & 20.6 & 26.8 & $<0.001$ \\
\hline Use of anti-hypertensive medication (\%) & 25.5 & 49.3 & 51.0 & $<0.001$ \\
\hline
\end{tabular}

Data are frequency (in \%), mean \pm SD or median [interquartile-range], as appropriate. Lowest C3 tertile ranged from 1.01 through $1.63 \mathrm{~g} / \mathrm{L}$, middle tertile from 1.64 through $1.94 \mathrm{~g} / \mathrm{L}$ and highest tertile from 1.95 through $3.35 \mathrm{~g} / \mathrm{L}$

The standardized value (z-score) for logHOMA2IR was positively and strongly associated with standardized C3 (standardized regression coefficient $\beta \quad(95 \%$ confidence interval (CI)) for HOMA2IR was 0.55 (0.48; 0.63), $\mathrm{p}<0.001)$. This means that for every $1 \mathrm{SD}$ increase in $\log H O M A 2 \mathrm{IR}$ there was a $0.55 \mathrm{SD}$ increase in 
circulating C3. The positive association between HOMA2IR and C3 was somewhat more pronounced in women than in men (Figure 6.1). This association remained significant after adjustment for age, sex, T2DM, CVD, use of medication, smoking, alcohol consumption, waist, and general inflammation $(\beta(95 \% \mathrm{CI})$ for HOMA2IR was $0.40(0.30 ; 0.49), \mathrm{p}<0.001$, Table 6.2). After adjustment for all these variables, HOMA2IR was also associated with the potential mediator, ALT $(\beta$ (95\% CI) for HOMA2IR $0.44(0.34 ; 0.54) \mathrm{p}<0.001)$. ALT was also associated with C3, after adjustment for the covariates listed above $(\beta$ (95\% CI) for ALT $0.25(0.17 ; 0.33)$ $\mathrm{p}<0.001)$ and remained so after additional adjustment for HOMA2IR (0.13 (0.05; $0.22) \mathrm{p}=0.002)$.

Table 6.2: Independent association between HOMA2IR and complement C3

\begin{tabular}{lllc}
\hline & & \multicolumn{1}{c}{ Complement C3 is outcome } \\
\cline { 3 - 4 } Model & & B HOMA $(95 \%$ CI $)$ & p-value \\
\hline 1 & Crude & $0.552(0.475 ; 0.629)$ & $<0.001$ \\
2 & Model 1 + age, sex & $0.564(0.488 ; 0.640)$ & $<0.001$ \\
3 & Model 2 + T2DM, CAD, use of medication & $0.544(0.462 ; 0.627)$ & $<0.001$ \\
4 & Model 3 + smoking, alcohol consumption & $0.534(0.450 ; 0.618)$ & $<0.001$ \\
5 & Model 4 + waist & $0.435(0.337 ; 0.533)$ & $<0.001$ \\
6 & Model 5 + general inflammation & $0.395(0.302 ; 0.487)$ & $<0.001$ \\
\hline
\end{tabular}

B: standardized regression coefficient represents the increase in circulating C3 (in SD) per 1 SD increase in log; HOMA2IR: adjusted for the variables mentioned in the different models

The associations between HOMA2IR and C3 reported above were attenuated by ALT regardless of additional adjustments for central obesity and/or inflammation (Table 6.3). In the full model (Table 6.3, Model 3) mediation by ALT was $14.2 \%$. Of note, in the final model (Table 6.3, Model $3+$ ALT), besides HOMA2IR and ALT also general inflammation, waist, sex and use of glucose- and lipid lowering medication were significantly associated with C3 while age, T2DM, CVD, use of anti-hypertensive medication, smoking and alcohol consumption were not independently associated with C3. 
Table 6.3: Association between HOMA2IR and C3 and the mediating role of ALT herein, independent of central obesity and inflammation

\begin{tabular}{lccc}
\hline & & C3 is outcome & \\
\cline { 2 - 3 } & B HOMA $(95 \%$ CI $)$ & p-value & Mediation ${ }^{\dagger}$ \\
\hline Model 1 & $0.534(0.450 ; 0.618)$ & $<0.001$ & n.a. \\
Model 1 + ALT & $0.465(0.371 ; 0.559)$ & $<0.001$ & $12.9 \%$ \\
Model 2 & $0.435(0.337 ; 0.533)$ & $<0.001$ & n.a. \\
Model 2 + ALT & $0.377(0.272 ; 0.482)$ & $<0.001$ & $13.3 \%$ \\
Model 3 & $0.395(0.302 ; 0.487)$ & $<0.001$ & n.a. \\
Model 3 + ALT & $0.339(0.241 ; 0.437)$ & $<0.001$ & $14.2 \%$ \\
\hline
\end{tabular}

Model 1: analyses were adjusted for age, sex, T2DM, CVD, use of medication, smoking and alcohol consumption (= model 4 from Table 6.2); Model 2: analyses were adjusted for age, sex, T2DM, CVD, use of medication, smoking, alcohol consumption and waist (= model 5 from Table 6.2); Model 3: analyses were adjusted for age, sex, T2DM, CVD, use of medication, smoking, alcohol consumption, waist and general inflammation score (= model 6 from Table 6.2);

B: standardized regression coefficient represents the increase in circulating C3 (in SD) per 1 SD increase in log HOMA2IR;

$\dagger$ indicates change in the magnitude of the change in regression coefficient $(\beta)$ after inclusion of ALT as compared to Models 1, 2, 3 without ALT, respectively (expressed in \%).

Since we observed that the association between HOMA2IR and C3 was more pronounced in women than in men (Figure 6.1), we also investigated whether the associations between HOMA2IR and C3, and the potential role of ALT herein, differed between men and women. Although the strength of the association between HOMA2IR and C3 was stronger in women $(\beta(95 \% \mathrm{CI})$ for HOMA2IR was 0.53 $(0.35 ; 0.70), \mathrm{p}<0.001$, adjusting for all above-mentioned covariates except sex) than in men $(\beta(95 \% \mathrm{CI})$ for HOMA2IR was $0.29(0.18 ; 0.40), \mathrm{p}<0.001)$, the attenuation of the strength of this association by ALT was similar between the sexes (women 13.9\% and men $15.2 \%)$. 


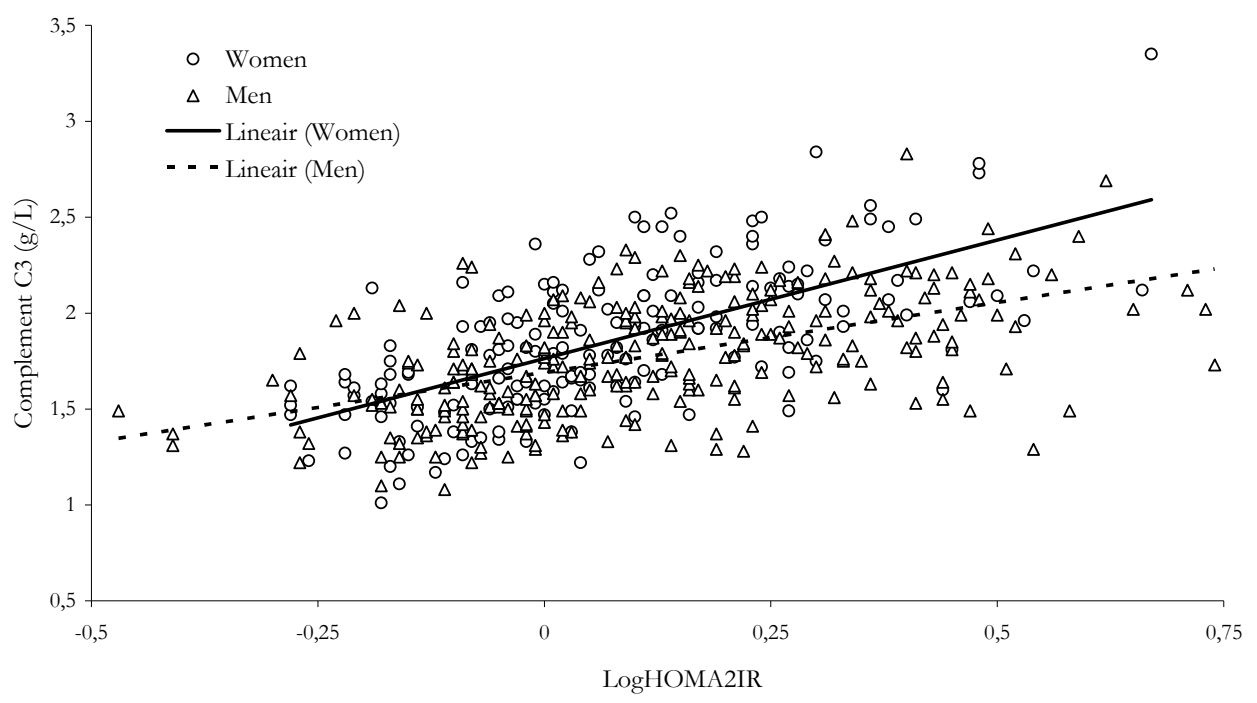

Figure 6.1: In the whole group, the linear regression coefficient $\beta$ for the $\mathrm{Z}$ score of $\log$ HOMA2IR was 0.55 (95\% CI $0.48 ; 0.63)(\mathrm{p}<0.001) \mathrm{n}=453)$. The association between HOMA2IR and C3 was stronger in women $(\beta$ was $0.78(95 \%$ CI $0.64 ; 0.92)(\mathrm{p}<0.001) \mathrm{n}=175)$ than in men $(\beta$ was $0.46(95 \%$ CI $0.37 ; 0.55)(\mathrm{p}<0.001) \mathrm{n}=278)$. This means that for every $1 \mathrm{SD}$ increase in $\log$ HOMA2IR, in men there was a $0.46 \mathrm{SD}$ increase in circulating $\mathrm{C} 3$, while in women the increase was a $0.78 \mathrm{SD}$ (crude associations).

\subsubsection{Additional analyses}

A more stringent cut-off value for alcohol consumption i.e. excluding all subjects who consumed $>20 \mathrm{~g} / \mathrm{d}$ of alcohol, has been proposed as a threshold to distinguish between non-alcoholic and alcoholic fatty liver disease [24]. We therefore repeated the analyses using this more stringent cut-off value. Again, a strong association was observed between HOMA2IR and C3 ( $n=381, \beta$ HOMA2IR was $0.56(0.47 ; 0.64)$, $\mathrm{p}<0.001)$. When ALT was included in the full model, $\beta$ for HOMA2IR decreased from $0.44(0.30 ; 0.52)$ to $0.34(0.24 ; 0.45))$ which equals $16.1 \%$ mediation by ALT.

It has been reported that plasma ALT levels may underestimate liver fat content in T2DM patients [30]. The analyses shown above were therefore also repeated without subjects with T2DM. When using the relaxed cut-off value for alcohol consumption, i.e. exclusion of women who consumed $>20 \mathrm{~g}$ and men who consumed $>40 \mathrm{~g}$ alcohol daily, the results were slightly attenuated compared to what was reported above; mediation by ALT was $8.8 \%(n=349)$. When using the more stringent cut-off value for alcohol consumption, i.e. exclusion of all subjects who consumed $>20 \mathrm{~g}$ alcohol daily, mediation by ALT was $9.5 \%(\mathrm{n}=290)$. 


\subsection{Discussion}

We investigated the mediating role of ALT -as a surrogate marker of fatty liver disease- in the association between insulin resistance (measured as HOMA2IR) and circulating concentrations of complement C3. Our results showed that after taking into account the effects of central obesity and low-grade inflammation and other risk factors, the strength of the association between insulin resistance and complement C3 was indeed additionally attenuated by ALT.

Insulin resistance and obesity-associated metabolic disorders are known to be associated with the amount of complement C3 in the circulation [6-9]. Our current data showed that the positive association between insulin resistance and C3 was independent of various metabolic and other risk factors. After adjustment for several covariates, including central obesity and inflammation, ALT attenuated the strength of this association by approximately $15 \%$. Of note, our finding that mediation by ALT occurred while taking into account general low-grade inflammation using an inflammation score that included CRP, IL6, SAA, sICAM, ceruloplasmin and haptoglobin suggests that this mediation neither represented nor was a consequence of a general inflammatory / acute phase response that is often observed in NAFLD [31], but was specifically related to C3.

The observed mediation suggests that fatty liver and concomitant liver dysfunction, as represented by ALT, may lead to an enhanced production of complement C3. Theoretically, increased amounts of circulating C3 may be due to either an increased production or to a decreased catabolism. However, C3 gene expression is increased in NASH [15], which is included in the NAFLD spectrum, and recent data suggest that, again in NASH, there is an increased rather than a decreased consumption of complement [32]. Therefore it is plausible that, in insulin resistance states, the production of $\mathrm{C} 3$ is increased to a level that (over)compensates for its consumption. There are several possible explanations for a mediating role of ALT. For instance, C3 production may be induced by accumulation of intracellular triglycerides and their metabolites in the hepatocytes. In insulin resistance there is an increased flux of fatty acids from the adipose tissue to the liver [33]. Fatty acids are not only substrate for triglyceride synthesis, but also act as second messengers that induce gene-expression [34]. The same holds true for intermediates of triglyceride synthesis such as diacylglycerols [35]. On the other hand, NAFLD is also associated with compromised cellular metabolism including hypoxia and reduced mitochondrial function which may affect both the (hepatic) C3 expression and its consumption via complement activation [36-38], although this may to some extent reflect general expression of acute phase proteins, rather than being specific for C3.

The final full model in this study explained approximately $40 \%$ of the association between insulin resistance and C3 ( $\beta$ for HOMA2IR was 0.55 in the crude association, compared to 0.34 in the full model), which leaves about $60 \%$ of this association 
currently unexplained. This remaining association can be related to, for instance, additional intrahepatic processes that are not reflected by plasma levels of ALT or to contribution of additional extrahepatic sources such as the vascular endothelium to circulating C3, that are not represented by the currently included covariates. Alternatively, it may be related to a direct effect of insulin on (hepatic) C3 expression and synthesis, that is independent of the intermediate mechanisms considered. This is to some extent corroborated by data showing that the increase in C3 in response to an oral fat load is more pronounced in insulin resistant (i.e. those with the metabolic syndrome) than in insulin sensitive (no metabolic syndrome) subjects [6] and is blunted by an increased post-prandial insulin surge that was induced by addition of glucose to the fat load [13].

A strength of this study is the detailed phenotyping of low-grade inflammation in all subjects, which allowed us to calculate a robust inflammation score that comprised information on 6 individual inflammation markers. Potential limitations lie in the fact that we used ALT levels as a measure of hepatic fat accumulation. However, even though ALT levels are not the ideal measure of hepatic fat accumulation, they can represent an acceptable measure for NALFD in epidemiological studies [27,39] and we did observe significant mediation by ALT, independent of general inflammation. We are aware that ALT levels may be affected by various factors that were not measured in our population, such as specific dietary factors and physical condition [40-42]. However, the effects of such factors will most likely lead to greater (random) variation in the ALT data, which would make our current finding that $\sim 14 \%$ of the association between insulin resistance and C3 is explained by ALT actually, if anything, more likely to be an underestimate of the true value. Other limitations may include that we measured total $\mathrm{C} 3$ concentration and not its activated compound C3a. However, it is the increased plasma concentration of C3 -rather than C3a- that has been identified as an important, independent risk factor for cardiovascular disease, hypertension and type 2 diabetes in cross-sectional as well as prospective studies [1-9], which was why we focused on complement C3 as the primary factor of interest in the current analyses. Lastly, the cross-sectional design of the study prohibits conclusions about causality, and the fact that the CODAM study population consists of middleaged Caucasian individuals with an increased T2DM and CHD risk profile may hinder direct extrapolation and to the general population. We did however adjust our analyses for potential confounders such as sex, smoking, presence of T2DM and CVD, use of medication, obesity and inflammation.

In conclusion, approximately $15 \%$ of the association between insulin resistance and circulating complement C3 was mediated by ALT. The mediation by ALT may be primarily related to increased hepatic $\mathrm{C} 3$ production in NAFLD. 


\subsection{Reference List}

1. Onat A, Hergenç G, Can G, Kaya Z, Yüksel H. Serum complement C3: a determinant of cardiometabolic risk, additive to the metabolic syndrome, in middle-aged population. Metabolism 2009 Nov 13 [Epub ahead of print].

2. Ajjan R, Grant PJ, Futers TS, Brown JM, Cymbalista CM, Boothby M et al. Complement C3 and $\mathrm{C}$-reactive protein levels in patients with stable coronary artery disease. Thromb Haemost. 2005: 94: 1048-1053.

3. Onat A, Uzunlar B, Hergenç G, Yazici M, Sari I, Uyarel H, Can G et al. Cross-sectional study of complement C3 as a coronary risk factor among men and women. Clin Sci (Lond). 2005: 108: 129-135.

4. Muscari A, Bozzoli C, Puddu GM, Sangiorgi Z, Dormi A, Rovinetti C et al. Association of serum C3 levels with the risk of myocardial infarction. Am J Med. 1995: 98: 357-364.

5. Engström G, Hedblad B, Janzon L, Lindgärde F. Complement C3 and C4 in plasma and incidence of myocardial infarction and stroke: a population-based cohort study. Eur J Cardiovasc Prev Rehabil. 2007:14: 392-397.

6. van Oostrom AJ, Alipour A, Plokker TW, Sniderman AD, Cabezas MC. The metabolic syndrome in relation to complement component 3 and postprandial lipemia in patients from an outpatient lipid clinic and healthy volunteers. Atherosclerosis. 2007: 190: 167-173.

7. Muscari A, Antonelli S, Bianchi G, Cavrini G, Dapporto S, Ligabue A et al. Serum C3 is a stronger inflammatory marker of insulin resistance than C-reactive protein, leukocyte count, and erythrocyte sedimentation rate: comparison study in an elderly population. Diabetes Care. 2007: 30: 2362-2368.

8. Engström G, Hedblad B, Berglund G, Janzon L, Lindgärde F et al. Plasma levels of complement C3 is associated with development of hypertension: a longitudinal cohort study. J Hum Hypertens. 2007: 21: 276-282.

9. Engström G, Hedblad B, Eriksson KF, Janzon L, Lindgärde F. Complement C3 is a risk factor for the development of diabetes: a population-based cohort study. Diabetes 2005: 54: 570-575.

10. Andrews E, Feldhoff P, Feldhoff R, Lassiter H. Comparative effects of cytokines and cytokine combinations on complement component C3 secretion by HepG2 cells. Cytokine. 2003: 2003: 23:164-169.

11. Stapp JM, Sjoelund V, Lassiter HA, Feldhoff RC, Feldhoff PW. Recombinant rat IL-1beta and IL-6 synergistically enhance C3 mRNA levels and complement component C3 secretion by H35 rat hepatoma cells. Cytokine. 2005: 30: 78-85.

12. Kawakami Y, Watanabe Y, Yamaguchi M, Sakaguchi H, Kono I, Ueki A. TNF-alpha stimulates the biosynthesis of complement $\mathrm{C} 3$ and factor B by human umbilical cord vein endothelial cells. Cancer Lett. 1997: 116: 21-26.

13. van Oostrom AJ, van Dijk H, Verseyden C, Sniderman AD, Cianflone K, Rabelink TJ et al. Addition of glucose to an oral fat load reduces postprandial free fatty acids and prevents the postprandial increase in complement component 3. Am J Clin Nutr. 2004: 79: 510-515. 
14. Alper CA, Johnson AM, Birtch AG, Moore FD. Human C'3: evidence for the liver as the primary site of synthesis. Science. 1969: 163: 286-288.

15. Sreekumar R, Rosado B, Rasmussen D, Charlton M. Hepatic gene expression in histologically progressive nonalcoholic steatohepatitis. Hepatology. 2003: 38: 244-251.

16. Bellentani S, Saccoccio G, Masutti F, Crocè LS, Brandi G, Sasso F et al. Prevalence of and risk factors for hepatic steatosis in Northern Italy. Ann Intern Med. 2000: 132: 112-117.

17. Jeong SK, Kim YK, Park JW, Shin YJ, Kim DS. Impact of visceral fat on the metabolic syndrome and nonalcoholic fatty liver disease. J Korean Med Sci. 2008: 23: 789-798.

18. Choy LN, Rosen BS, Spiegelman BM. Adipsin and an endogenous pathway of complement from adipose cells. J Biol Chem. 1992: 267: 12736-12741.

19. Peake PW, O'Grady S, Pussell BA, Charlesworth JA. Detection and quantification of the control proteins of the alternative pathway of complement in 3T3-L1 adipocytes. Eur J Clin Invest. 1997: 27: 922-927.

20. Muscari A, Sbano D, Bastagli L, Poggiopollini G, Tomassetti V, Forti P et al. Effects of weight loss and risk factor treatment in subjects with elevated serum C3, an inflammatory predictor of myocardial infarction. Int J Cardiol. 2005: 100: 217-223.

21. Engström G, Hedblad B, Janzon L, Lindgärde F. Weight gain in relation to plasma levels of complement factor 3: results from a population-based cohort study. Diabetologia 2005: 48: 2525-2531.

22. Kruijshoop M, Feskens EJ, Blaak EE, de Bruin TW. Validation of capillary glucose measurements to detect glucose intolerance or type 2 diabetes mellitus in the general population. Clin Chim Acta. 2004: 341: 33-40.

23. van Dam RM, Boer JM, Feskens EJ, Seidell JC. Parental history of diabetes modifies the association between abdominal adiposity and hyperglycemia. Diabetes Care. 2001: 24: 14541459.

24. Neuschwander-Tetri BA, Caldwell SH. Nonalcoholic steatohepatitis: summary of an AASLD Single Topic Conference. Hepatology. 2003: 37: 1202-1209.

25. Simera I, Moher D, Hoey J, Schulz KF, Altman DG. A catalogue of reporting guidelines for health research. Eur J Clin Invest. 2010: 40: 35-53.

26. Levy J, Matthews DR, Hermans MP. Correct homeostasis model assessment (HOMA) evaluation uses the computer program (Letter). Diabetes Care 1998: 21: 2191-2192.

27. Brouwers MC, Cantor RM, Kono N, Yoon JL, van der Kallen CJ, Bilderbeek-Beckers MA et al. Heritability and genetic loci of fatty liver in familial combined hyperlipidemia. J Lipid Res. 2006: 47: 2799-2807.

28. Grundy SM, Cleeman JI, Daniels SR, Donato KA, Eckel RH, Franklin BA et al. Diagnosis and management of the metabolic syndrome: an American Heart Association/National Heart, Lung, and Blood Institute Scientific Statement. Circulation. 2005: 112: 2735-2752.

29. Ocké MC, Bueno-de-Mesquita HB, Goddijn HE, Jansen A, Pols MA, van Staveren WA, et al. The Dutch EPIC food frequency questionnaire. I. Description of the questionnaire, and relative validity and reproducibility for food groups. Int J Epidemiol. 1997: 26: S37-S48. 
30. Kotronen A, Juurinen L, Hakkarainen A, Westerbacka J, Cornér A, Bergholm R et al. Liver fat is increased in type 2 diabetic patients and underestimated by serum alanine aminotransferase compared with equally obese nondiabetic subjects. Diabetes Care. 2008: 31: 165-169.

31. Tilg $\mathrm{H}$, Moschen AR. Insulin resistance, inflammation, and non-alcoholic fatty liver disease. Trends Endocrinol Metab. 2008: 19: 371-379.

32. Rensen SS, Slaats Y, Driessen A, Peutz-Kootstra CJ, Nijhuis J, Steffensen R et al. Activation of the complement system in human nonalcoholic fatty liver disease. Hepatology. 2009: 50: 18091917.

33. Donnelly KL, Smith CI, Schwarzenberg SJ, Jessurun J, Boldt MD, Parks EJ. Sources of fatty acids stored in liver and secreted via lipoproteins in patients with nonalcoholic fatty liver disease. J Clin Invest. 2005: 115: 1343-1351.

34. Staudinger JL, Lichti K. Cell signaling and nuclear receptors: new opportunities for molecular pharmaceuticals in liver disease. Mol Pharm. 2008: 5: 17-24.

35. Newton AC: Lipid activation of protein kinases. J Lipid Res. 2009: 50: S266-S271.

36. Mantena SK, Vaughn DP, Andringa KK, Eccleston HB, King AL, Abrams GA et al. High fat diet induces dysregulation of hepatic oxygen gradients and mitochondrial function in vivo. Biochem J. 2009: 417: 183-193.

37. Wenger RH, Rolfs A, Marti HH, Bauer C, Gassmann M. Hypoxia, a novel inducer of acute phase gene expression in a human hepatoma cell line. J Biol Chem. 1995: 270: 27865-27870.

38. Collard CD, Agah A, Stahl GL. Complement activation following reoxygenation of hypoxic human endothelial cells: role of intracellular reactive oxygen species, NF-kappaB and new protein synthesis. Immunopharmacology. 1998: 39: 39-50.

39. Westerbacka J, Cornér A, Tiikkainen M, Tamminen M, Vehkavaara S, Häkkinen AM, et al. Women and men have similar amounts of liver and intra-abdominal fat, despite more subcutaneous fat in women: implications for sex differences in markers of cardiovascular risk. Diabetologia. 2004: 47: 1360-1369.

40. Frelut ML, Emery-Fillon N, Guilland JC, Dao HH, de Courcy GP. Alanine amino transferase concentrations are linked to folate intakes and methylenetetrahydrofolate reductase polymorphism in obese adolescent girls. J.Pediatr Gastroenterol Nutr. 2006: 43: 234-239.

41. Le Couteur DG, Blyth FM, Creasey HM, Handelsman DJ, Naganathan V, Sambrook PN, et al. The association of alanine transaminase with aging, frailty, and mortality. J Gerontol A Biol Sci Med Sci. 2010: 65: 712-717.

42. Nosaka K, Clarkson PM, Apple FS. Time course of serum protein changes after strenuous exercise of the forearm flexors. J Lab Clin Med. 1992: 119: 183-188. 



\title{
Chapter 7
}

\author{
Summary and Discussion
}





\subsection{The CODAM Study}

The primary aims of the Cohort on Diabetes and Atherosclerosis Maastricht (CODAM) study are to evaluate the progression of atherosclerosis and cardiovascular disease (CVD) in subjects with a relatively high risk of type 2 diabetes mellitus (T2DM) and to evaluate the development of T2DM in subjects who were selected for specific T2DM risk factors. To be able to study these questions the cohort will be seen every 7 years to assure a regular follow-up. This thesis focused on the first primary aim. Since only the baseline measurements of this cohort were available by the time the investigations described in this thesis were done, data from crosssectional analyses are presented.

\subsection{The metabolic syndrome in CODAM}

Presence of the metabolic syndrome is a "common denominator" in subjects with high risk of CVD and T2DM [1-4]. The metabolic syndrome defines a clustering of risk factors including abdominal obesity, hypertension, elevated plasma triglyceride concentration, reduced high-density lipoprotein (HDL)-cholesterol concentration and elevated fasting blood glucose levels [5, 6]. Given the selection criteria for the CODAM study, the metabolic syndrome was likely to be a frequent finding in CODAM participants. Prevalence of the metabolic syndrome according to national cholesterol education program 2005 [7] was in subjects with normal glucose tolerance $33.2 \%$, with impaired glucose metabolism $69.3 \%$ and, with T2DM $86.3 \%$ in the CODAM participants. Cross-sectional and prospective studies have shown that the metabolic syndrome is associated with CVD and that people with the metabolic syndrome have an increased risk for future CVD [8-11]. However the exact mechanisms that might explain this association are not entirely clear. Therefore we investigated cross-sectionally which mechanisms/processes might underlie the association between the metabolic syndrome and CVD [12-14]. In these investigations we focused on several metabolic markers and potential intermediates that are of specific interest in the relation between the metabolic syndrome and CVD, including insulin resistance, inflammation, endothelial dysfunction, complement C3 and nonalcoholic fatty liver disease (NAFLD).

\subsection{Central and peripheral artery disease in CODAM}

\subsubsection{The metabolic syndrome and coronary and peripheral artery disease}

We first set out to investigate how the association between the metabolic syndrome and different aspects of cardiovascular disease were affected by intermediate processes 
in CVD, i.e. inflammation and endothelial dysfunction. In these investigations we specifically focused on two distinct aspect of CVD i.e. the coronary and the peripheral vasculature, since these are two different vascular beds that might be affected in a different manner by the metabolic syndrome.

We showed that low-grade inflammation explained, statistically, part of the association between the metabolic syndrome and coronary artery disease (CAD) (up to 26\%) and the severity of peripheral artery disease (PAD) (up to 29\%, Chapter 2). The most studied marker of systemic low-grade inflammation in association with the metabolic syndrome and CVD is C-reactive protein $[15,16]$. However, more markers of inflammation exist, including interleukin 6, soluble intercellular adhesion molecules, soluble vascular cell adhesion molecules and serum amyloid $A$. These have been investigated before [15, 17-19], but only as separate markers. In our investigations we combined several inflammatory markers into a composed score that represented systemic low-grade inflammation what is a more robust measure for inflammation than the markers separately with the additional advantage of less misclassification [20]. Since the association between the metabolic syndrome and CAD and the severity of PAD was only partly explained by inflammation we subsequently investigated (Chapter 3) whether endothelial dysfunction could explain an additional part of this association. As a measure for endothelial dysfunction we also used a robust measure of biomarkers that were combined into an endothelial dysfunction score [20]. We found that endothelial dysfunction explained about $19 \%$ of the association between the metabolic syndrome and the severity of PAD, low-grade inflammation explained $28 \%$. Together endothelial dysfunction and low-grade inflammation explained $36 \%$, so $8 \%$ was additional to low-grade inflammation (Figure 7.1). This implies that inflammation and endothelial dysfunction partly represent the same pathway in the relation between the metabolic syndrome and PAD but can have individual contributions as well. On the other hand, endothelial dysfunction did not explain any part of the association between the metabolic syndrome and CAD. Together with the former this shows that the pathogenesis of peripheral and coronary artery disease differ in this respect. It could be argued that it would have been better to use functional measure of endothelial dysfunction, like flow-mediated dilatation instead of biomarkers [21]. However compared to such a more direct measure of endothelial function, the use of biomarkers would only underestimate our findings which make our results unlikely to be a spurious finding. 


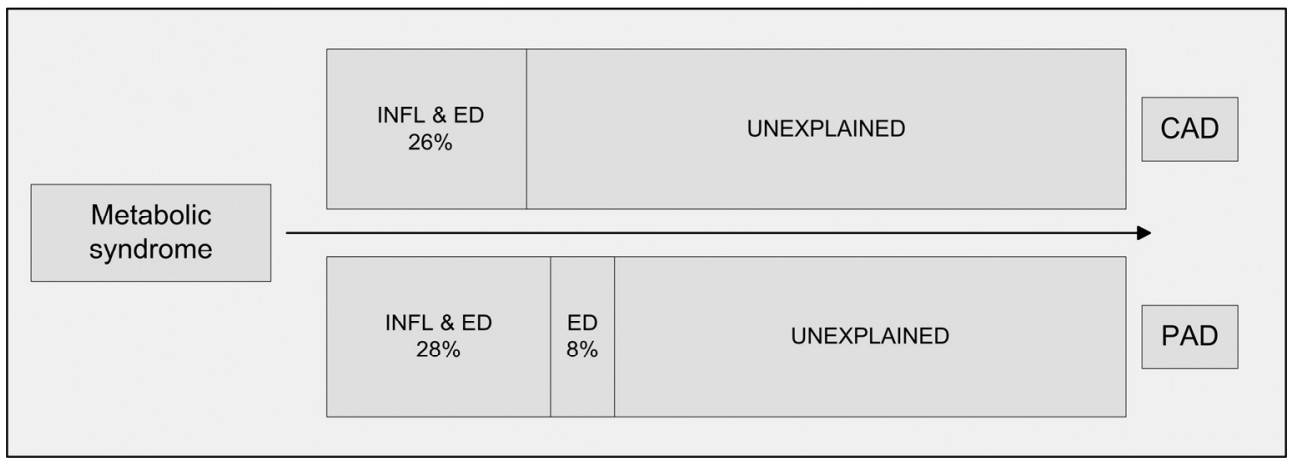

Figure 7.1: The association between the metabolic syndrome and coronary artery disease (CAD) and peripheral artery disease (PAD), adjusted for age, sex and lifetime smoking, is partly mediated by inflammation (INFL) and endothelial dysfunction (ED)

\subsubsection{The complement system and coronary heart disease}

The complement factor $\mathrm{C} 3$ has been established as a cardiovascular risk factor in both cross-sectional and longitudinal studies $[22,23]$ and is substantially increased in the circulation of subjects with the metabolic syndrome [24].

In the CODAM population (Chapter 4), we confirmed that human serum complement factor $\mathrm{C} 3$ was indeed cross-sectionally associated with coronary heart disease (CHD) as was previously shown by others [25-27]. However, based on previous in vitro data that cigarette smoke can activate the complement system by modifying complement C3 [28-30] we hypothesized that smoking may enhance the effect of complement of the risk of CVD. Indeed, closer examination of the association between complement $\mathrm{C} 3$ and $\mathrm{CHD}$ learned that this was mainly present in the group of heavy smokers. This association was independent of cardiometabolic risk factors like insulin resistance, systemic inflammation and the metabolic syndrome. The overall association of $\mathrm{C} 3$ with $\mathrm{CHD}$ we found in the whole CODAM population corresponds with the associations between $\mathrm{C} 3$ and prevalence of $\mathrm{CHD}$, independent of age, sex and some additional risk factors reported by several other studies [23, 25, 31, 32]. There are several possible explanations how smoking might increase the risk of CVD associated with C3 in these cross-sectional evaluations. A first explanation is that the activation of complement C3 by (heavy) smoking might be one of the mechanisms by which smoking can enhance the process of inflammation in the atherosclerotic plaque and thereby increasing the risk of CVD. Another possibility is that smoking would not directly affect the complement system but rather induce oxidative stress and inflammation in the atherosclerotic plaque, which might affect the expression and/or activation of the complement system. 


\subsection{Metabolic risk factors in CODAM}

\subsubsection{The metabolic syndrome and non-alcoholic fatty liver disease}

Non-alcoholic fatty liver disease is a highly prevalent finding (70\% to $80 \%)$ in subjects with the metabolic syndrome $[33,34]$ and has a wide histological spectrum ranging from simple steatosis and non-alcoholic steatohepatitis to more progressive forms such as fibrosis and eventually cirrhosis. Alanine aminotransferase (ALT) in the circulation is a simple measure for fatty liver disease. Several metabolic syndromerelated processes might affect the development of NAFLD.

We showed in Chapter 5 that insulin resistance was, statistically, the strongest mediator of the association between the metabolic syndrome and ALT while other metabolic syndrome related processes like inflammatory adipokines, endothelial dysfunction and non-esterified fatty acids (NEFA) also explained a part of the association, but to a lesser extent, Figure 7.2. The strong mediation by insulin resistance was not really surprising because of well-known relations between the metabolic syndrome, insulin resistance and steatosis [35]. The mediation by inflammatory adipokines and endothelial dysfunction did not add to the mediation by insulin resistance, which implies that these processes overlap and supports the notion that -in the metabolic syndrome- insulin resistance-associated adipose tissue inflammation and endothelial dysfunction might actually contribute to the development and/or progression of NAFLD, as reflected by ALT. NEFA on the other hand did add to the mediation by insulin resistance; about half of the mediation by NEFA was additional to insulin resistance. This suggests that half of the mediation by NEFA may occur via pathways included in the process of adipose tissue inflammation and insulin signaling, while the other half occurs via other processes like dietary fatty acids and direct uptake by hepatocytes of adipose tissue derived NEFA.

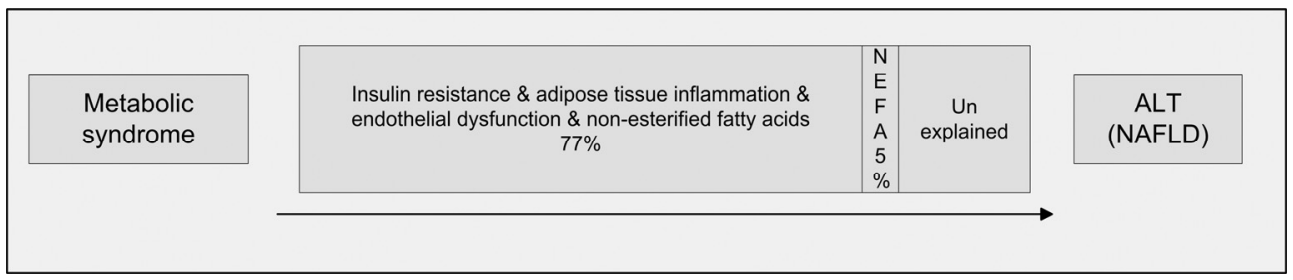

Figure 7.2: The association between the metabolic syndrome and non-alcoholic fatty liver disease (NAFLD; measured as ALT), adjusted for age, sex, smoking, alcohol consumption and the use of lipid-lowering and anti-hypertensive medication, is for a very large part mediated by insulin resistance, with no independent contribution of adipose tissue inflammation, endothelial dysfunction. The mediation by non-esterified fatty acids (NEFA) is partly independent of insulin resistance 


\subsubsection{Fatty liver as an intermediate in the relation between insulin resistance and complement $\mathrm{C3}$}

As described above (paragraph 7.3.2), increased levels of circulating complement C3 are a frequent finding in subjects with the metabolic syndrome. Several aspects of the metabolic syndrome, including insulin resistance, may contribute to increased amounts of circulating complement factor $\mathrm{C} 3$.

In the CODAM population, insulin resistance was, as expected based on literature [36, 37], indeed associated with circulating concentrations of complement C3. We now showed that NAFLD, measured by ALT, partly explained this association, independent of obesity and low-grade inflammation (Chapter 6). Given the substantial production of $\mathrm{C} 3$ by the liver, the observed 14\% mediation appeared, at first sight, to be quite low. However estimations of the contribution of the liver to systemic C3 are primarily based on data from subjects who received a liver transplantation [38] and such individuals can metabolically not be compared to the subjects in the CODAM study. Moreover, we did not study the association between ALT and circulating C3 but evaluated to what extent ALT could explain the association between C3 and insulin resistance.

Taking all the above together, our studies showed that several processes explain part of the association between the metabolic syndrome and (metabolic intermediates of) cardiovascular disease. These processes include low-grade inflammation, insulin resistance, endothelial dysfunction, complement and non-esterified fatty acids, but our investigations showed that these processes overall are partly overlapping and partly independent from each other.

\subsection{Methodological considerations}

\subsubsection{Internal validity}

In the manuscripts presented in this thesis, we have used a range of biomarkers as proxy measures of general inflammation, adipose tissue inflammation and endothelial dysfunction. Different sets of markers were combined into scores to obtain more robust measures of these above-mentioned processes with the benefit of less misclassification and reduction of the problem of multiple testing. An important advantage of the use of biomarkers is that they are relatively easy obtainable in large epidemiological cohorts. A disadvantage might be that they are estimates of underlying (patho-)physiological processes but do not directly measure them.

We have used several markers for low-grade inflammation and made a distinction between general and adipose tissue-related low-grade inflammation. C-reactive protein 
is a marker for inflammation that is mostly produced by the liver and therefore a marker for general inflammation. Adiponectin and leptin on the other hand are markers that are exclusively produced by adipocytes and therefore representative for adipose-tissue related inflammation. The other markers we used (i.e. IL6, sICAM, SAA) are not unique produced in one cell type. So it is more difficult for these markers to assign them to a specific type of inflammation and therefore it is possible that both inflammation scores include markers that are not unique for that score.

We also used biomarkers to estimate endothelial dysfunction. Functional measures for endothelial function have been used in other studies, however until now there is not a gold standard to measure endothelial dysfunction. The most used functional measure is endothelium-dependent flow-mediated vasodilatation. Unfortunately, this measure was not available. By using a combined score for endothelial dysfunction instead of one or more individual markers we, again, tried to reduce the problem of misclassification and multiple testing.

The gold standard for diagnosing and staging NAFLD is a liver biopsy. This is an invasive procedure with risk of postinterventional bleeding and is therefore ethically not acceptable without clinical indication. Other techniques that are frequently used in epidemiological studies include imaging techniques like ultrasound or by measurement of circulating markers such as alanine-aminotransferase. However, previous studies have shown that ALT is a reasonable predictor of NAFLD as detected by ultrasound $[39,40]$.

Coronary artery disease was defined as self-reported myocardial infarction, bypass surgery of the coronary arteries, balloon dilatation or stent placement (through questionnaires) and/or the presence of signs of myocardial infarction or ischemia on a 12-lead electrocardiogram. For more than $75 \%$ of the subjects, the self-reported CAD could be confirmed using available hospital registries. The ankle-arm index was measured for PAD, the most used method. Since only 28 of 574 subjects had an ankle-arm index (AAIx) $<0.9$ (the traditional criterion to define PAD), we lacked power to use PAD as a categorical variable and we therefore used the AAIx as a continuous measure for the severity of PAD.

\subsubsection{External validity}

Subjects who were selected into CODAM had a relatively high risk of T2DM and CVD. Generalizability of our current data is therefore restricted to subjects who have a comparable risk profile of obesity, (slightly) disturbed glucose metabolism and/or high blood pressure. Subjects who fulfill these criteria are present in our Western society with a high prevalence and they are the ones who have the highest risk to develop cardiovascular and/or macrovascular complications. The data presented in this thesis can largely be generalized to those high-risk subjects and these data contribute to our understanding of development of disease in this particular group of 
subjects. We should, however, be cautious with generalization of our results to highrisk subjects of different ethnicity or of (much) younger age, since these were not included in our cohort.

For more generalizable data, our investigations should be repeated in other populations.

All studies presented in this thesis were done cross-sectionally which precludes conclusions on causality.

\subsection{Future research within CODAM}

From 2005 until 2009 all participants of the CODAM baseline measurement were invited to participate in the first follow-up evaluation (CODAM 2). Of the original 574 participants, 491 (>85\%) visited our laboratory twice for these 7 years follow-up evaluations. During these visits all measurements that were performed during CODAM 1 were repeated to assure that longitudinal analysis can be performed.

During the first visit anthropometric measures were taken (length, weight, waist and hip circumference, skin-fold measures) and blood pressure was measured in both arms in sitting and in supine position. Besides in the arms, blood pressure was also measured in both legs, to be able to calculate the ankle-arm index. In supine position also an ECG was taken so signs of CAD could be recognized. Then fasting blood samples were taken to measure markers like among others glucose, HDL-cholesterol, triglycerides, markers of low-grade inflammation, complement C3 and endothelial dysfunction. To define whether the participants had T2DM we performed an oral glucose tolerance test, during which the participants completed the same questionnaires as were filled in during CODAM 1.

The second visit was mainly used to perform vascular measurements and additional measurements on body composition and fat distribution. Ultrasound was used to measure intima-media thickness in both carotid arteries. Also the distension was measured in both carotid arteries as well as in the brachial and femoral arteries, as a measure of stiffness. Other measures of stiffness were the augmentation index and pulse wave velocity (central: carotid artery - femoral artery and peripheral: femoral artery - ankle). Since in CODAM 1 we only had the biomarker ALT as a measure for NAFLD, we now used ultrasound to have a more direct/better measure for fatty liver disease. Finally an intravenous glucose tolerance test was done.

With the results of CODAM 2 together with those of CODAM 1, we will be able to study various aspects and causes of the development and progression of T2DM, atherosclerosis and CVD in these high-risk subjects. 


\subsection{References}

1 Malik S, Wong ND, Franklin SS, et al. Impact of the metabolic syndrome on mortality from coronary heart disease, cardiovascular disease, and all causes in United States adults. Circulation 2004;110(10):1245-1250.

2 McNeill AM, Rosamond WD, Girman CJ, et al. The metabolic syndrome and 11-year risk of incident cardiovascular disease in the atherosclerosis risk in communities study. Diabetes Care 2005;28(2):385-390.

3 Wannamethee SG. The metabolic syndrome and cardiovascular risk in the British Regional Heart Study. Int J Obes (Lond) 2008;32 Suppl 2(S25-29.

4 Wilson PW, Meigs JB. Cardiometabolic risk: a Framingham perspective. Int J Obes (Lond) 2008;32 Suppl 2(S17-20.

5 Alberti KG, Eckel RH, Grundy SM, et al. Harmonizing the metabolic syndrome: a joint interim statement of the International Diabetes Federation Task Force on Epidemiology and Prevention; National Heart, Lung, and Blood Institute; American Heart Association; World Heart Federation; International Atherosclerosis Society; and International Association for the Study of Obesity. Circulation 2009;120(16):1640-1645.

6 Reaven GM. Banting lecture 1988. Role of insulin resistance in human disease. Diabetes 1988;37(12):1595-1607.

7 Grundy SM, Cleeman JI, Daniels SR, et al. Diagnosis and management of the metabolic syndrome: an American Heart Association/National Heart, Lung, and Blood Institute Scientific Statement. Circulation 2005;112(17):2735-2752.

8 Alexander CM, Landsman PB, Teutsch SM, et al. NCEP-defined metabolic syndrome, diabetes, and prevalence of coronary heart disease among NHANES III participants age 50 years and older. Diabetes 2003;52(5):1210-1214.

9 Hunt KJ, Williams K, Hazuda HP, et al. The metabolic syndrome and the impact of diabetes on coronary heart disease mortality in women and men: the San Antonio Heart Study. Ann Epidemiol 2007;17(11):870-877.

10 Olijhoek JK, van der Graaf Y, Banga JD, et al. The metabolic syndrome is associated with advanced vascular damage in patients with coronary heart disease, stroke, peripheral arterial disease or abdominal aortic aneurysm. Eur Heart J 2004;25(4):342-348.

11 Pischon T, Hu FB, Rexrode KM, et al. Inflammation, the metabolic syndrome, and risk of coronary heart disease in women and men. Atherosclerosis 2008;197(1):392-399.

12 Jacobs M, van Greevenbroek MM, van der Kallen CJ, et al. The association between the metabolic syndrome and peripheral, but not coronary, artery disease is partly mediated by endothelial dysfunction: the CODAM study. Eur J Clin Invest 41(2):167-175.

13 Jacobs M, van Greevenbroek MM, van der Kallen CJ, et al. Low-grade inflammation can partly explain the association between the metabolic syndrome and either coronary artery disease or severity of peripheral arterial disease: the CODAM study. Eur J Clin Invest 2009;39(6):437-444. 
van Greevenbroek MM, Jacobs M, van der Kallen CJ, et al. Human plasma complement C3 is independently associated with coronary heart disease, but only in heavy smokers (the CODAM study). Int J Cardiol

15 Kressel G, Trunz B, Bub A, et al. Systemic and vascular markers of inflammation in relation to metabolic syndrome and insulin resistance in adults with elevated atherosclerosis risk. Atherosclerosis 2009;202(1):263-271.

$16 \mathrm{Vu} \mathrm{JD}, \mathrm{Vu} \mathrm{JB}$, Pio JR, et al. Impact of C-reactive protein on the likelihood of peripheral arterial disease in United States adults with the metabolic syndrome, diabetes mellitus, and preexisting cardiovascular disease. Am J Cardiol 2005;96(5):655-658.

17 Bataille R, Klein B. C-reactive protein levels as a direct indicator of interleukin-6 levels in humans in vivo. Arthritis Rheum 1992;35(8):982-984.

18 Haught WH, Mansour M, Rothlein R, et al. Alterations in circulating intercellular adhesion molecule-1 and L-selectin: further evidence for chronic inflammation in ischemic heart disease. Am Heart J 1996;132(1 Pt 1):1-8.

19 Leinonen E, Hurt-Camejo E, Wiklund O, et al. Insulin resistance and adiposity correlate with acute-phase reaction and soluble cell adhesion molecules in type 2 diabetes. Atherosclerosis 2003;166(2):387-394.

20 Schram MT, Chaturvedi N, Schalkwijk C, et al. Vascular risk factors and markers of endothelial function as determinants of inflammatory markers in type 1 diabetes: the EURODIAB Prospective Complications Study. Diabetes Care 2003;26(7):2165-2173.

21 Henry RM, Ferreira I, Dekker JM, et al. The metabolic syndrome in elderly individuals is associated with greater muscular, but not elastic arterial stiffness, independent of low-grade inflammation, endothelial dysfunction or insulin resistance-The Hoorn Study. J Hum Hypertens 2009;

22 Engstrom G, Hedblad B, Janzon L, et al. Complement C3 and C4 in plasma and incidence of myocardial infarction and stroke: a population-based cohort study. Eur J Cardiovasc Prev Rehabil 2007;14(3):392-397.

23 Muscari A, Bozzoli C, Puddu GM, et al. Association of serum C3 levels with the risk of myocardial infarction. Am J Med 1995;98(4):357-364.

24 van Oostrom AJ, Alipour A, Plokker TW, et al. The metabolic syndrome in relation to complement component 3 and postprandial lipemia in patients from an outpatient lipid clinic and healthy volunteers. Atherosclerosis 2007;190(1):167-173.

25 Ajjan R, Grant PJ, Futers TS, et al. Complement C3 and C-reactive protein levels in patients with stable coronary artery disease. Thromb Haemost 2005;94(5):1048-1053.

26 Carter AM, Prasad UK, Grant PJ. Complement C3 and C-reactive protein in male survivors of myocardial infarction. Atherosclerosis 2009;203(2):538-543.

27 Onat A, Uzunlar B, Hergenc G, et al. Cross-sectional study of complement C3 as a coronary risk factor among men and women. Clin Sci (Lond) 2005;108(2):129-135.

28 Kew RR, Ghebrehiwet B, Janoff A. Characterization of the third component of complement (C3) after activation by cigarette smoke. Clin Immunol Immunopathol 1987;44(2):248-258. 
29 Perricone R, de Carolis C, de Sanctis G, et al. Complement activation by cigarette smoke condensate and tobacco infusion. Arch Environ Health 1983;38(3):176-179.

30 Robbins RA, Nelson KJ, Gossman GL, et al. Complement activation by cigarette smoke. Am J Physiol 1991;260(4 Pt 1):L254-259.

31 Szeplaki G, Prohaszka Z, Duba J, et al. Association of high serum concentration of the third component of complement (C3) with pre-existing severe coronary artery disease and new vascular events in women. Atherosclerosis 2004;177(2):383-389.

32 Verseyden C, Meijssen S, van Dijk H, et al. Effects of atorvastatin on fasting and postprandial complement component 3 response in familial combined hyperlipidemia. J Lipid Res 2003;44(11):2100-2108.

33 Bellentani S, Saccoccio G, Masutti F, et al. Prevalence of and risk factors for hepatic steatosis in Northern Italy. Ann Intern Med 2000;132(2):112-117.

34 Jeong SK, Kim YK, Park JW, et al. Impact of visceral fat on the metabolic syndrome and nonalcoholic fatty liver disease. J Korean Med Sci 2008;23(5):789-795.

35 Yki-Jarvinen $\mathrm{H}$, Westerbacka $\mathrm{J}$. The fatty liver and insulin resistance. Curr Mol Med 2005;5(3):287-295.

36 Engstrom G, Hedblad B, Eriksson KF, et al. Complement C3 is a risk factor for the development of diabetes: a population-based cohort study. Diabetes 2005;54(2):570-575.

37 Muscari A, Antonelli S, Bianchi G, et al. Serum C3 is a stronger inflammatory marker of insulin resistance than $\mathrm{C}$-reactive protein, leukocyte count, and erythrocyte sedimentation rate: comparison study in an elderly population. Diabetes Care 2007;30(9):2362-2368.

38 Alper CA, Johnson AM, Birtch AG, et al. Human C'3: evidence for the liver as the primary site of synthesis. Science 1969;163(864):286-288.

39 Brouwers MC, Cantor RM, Kono N, et al. Heritability and genetic loci of fatty liver in familial combined hyperlipidemia. J Lipid Res 2006;47(12):2799-2807.

40 Siegelman ES, Rosen MA. Imaging of hepatic steatosis. Semin Liver Dis 2001;21(1):71-80. 
Samenvatting 

Het metabool syndroom is een verschijnsel dat vaak voorkomt bij mensen met een hoog risico op type 2 diabetes mellitus en hart- en vaatziekten en is een cluster van risicofactoren, waaronder abdominale obesitas, hoge bloeddruk, verhoogde concentratie van triglyceriden en verlaagde concentratie van HDL-cholesterol in het bloed en verhoogde bloedglucose waardes. Als gevolg van de selectiecriteria die gebruikt zijn om te komen tot onze onderzoekspopulatie (Cohort Onderzoek naar Diabetes en Atherosclerose Maastricht; CODAM), werd het metabool syndroom vaak gevonden bij de deelnemers. Het metabool syndroom kwam bij 33.2\% voor van de personen met een normale glucose tolerantie, bij 69.3\% van de personen met een verminderde glucose tolerantie en $86.3 \%$ bij personen met type 2 diabetes.

Diverse andere studies hebben al aangetoond dat het metabool syndroom geassocieerd is met hart- en vaatziekten en dat personen met het metabool syndroom ook een verhoogd risico hebben op hart- en vaatziekten in de toekomst. De precieze mechanismen die deze associatie kunnen verklaren zijn nog niet bekend. Daarom hebben wij gekeken naar de mechanismen/processen die hier aan ten grondslag kunnen liggen en hebben ons hierbij gefocust op insuline resistentie, ontsteking, endotheel dysfunctie, complement C3 en niet-alcoholische leververvetting.

In hoofdstuk 2 en 3 hebben we gekeken naar de associatie van het metabool syndroom met hart- en vaatziekten en hoe deze mogelijk verklaard kan worden. We hebben ons gefocussed op twee verschillende aspecten van hart- en vaatziekten, namelijk coronair vaatlijden en perifeer vaatlijden omdat deze twee vaatbedden waarschijnlijk op een andere manier door het metabool syndroom beïnvloed worden.

We laten in hoofdstuk 2 zien dat laaggradige ontsteking statistisch gezien een deel kan verklaren van de associatie die gevonden is tussen het metabool syndroom en coronair vaatlijden (tot $26 \%$ ) en van de associatie tussen het metabool syndroom en de ernst van perifeer vaatlijden (tot wel 29\%). Diverse markers die in het bloed gemeten kunnen worden geven een maat voor laaggradige ontsteking, maar er is niet één marker die 'uniek' is. Daarom hebben we in ons onderzoek meerdere markers gebruikt voor het berekenen van een gecombineerde score die representatief is voor algehele laaggradige ontsteking. Deze score is een robuustere maat voor ontsteking dan de afzonderlijke markers met tevens het voordeel dat de kans op misclassificatie veel kleiner is.

Aangezien laaggradige ontsteking de associatie tussen het metabool syndroom en verschillende aspecten van hart- en vaatziekten slechts voor een deel kan verklaren hebben we in hoofdstuk 3 gekeken of endotheel dysfunctie een extra deel van deze associatie kan verklaren. Voor endotheel dysfunctie hebben we, net als voor laaggradige ontsteking, een gecombineerde score berekend van diverse markers. Endotheel dysfunctie kon 19\% en laaggradige ontsteking $28 \%$ van de associatie tussen het metabool syndroom en de ernst van perifeer vaatlijden verklaren. Samen 
verklaarden ze $36 \%$, dus endotheel dysfunctie voegde $8 \%$ toe aan de verklaring die gevonden werd door laaggradige ontsteking. Het lijkt er dus op dat laaggradige ontsteking en endotheel dysfunctie deels dezelfde route representeren, maar ook ieder hun eigen bijdrage hebben. Verder bleek dat endotheel dysfunctie niets verklaard van de associatie tussen het metabool syndroom en coronair vaatlijden. Dit laat, samen met het voorgaande, zien dat de pathogenese van perifeer en coronair vaatlijden verschillend is.

In hoofdstuk 4 hebben we laten zien dat complement C3 cross-sectioneel geassocieerd is met coronair vaatlijden. Dit hebben we uitgebreider bekeken en zagen dat deze associatie voornamelijk naar voren kwam in de groep personen die veel rookt, onafhankelijk van cardiometabole risicofactoren zoals insuline resistentie, laaggradige ontsteking en het metabool syndroom.

Niet-alcoholische leververvetting in verschillende gradaties wordt vaak gezien bij mensen met het metabool syndroom. Omdat leververvetting niet heel eenvoudig vastgesteld kan worden, hebben wij daarvoor de niveaus van alanine aminotransferase (ALT) in het bloed bepaald wat een vereenvoudigde maat is voor niet-alcoholische leververvetting. We laten in hoofdstuk 5 zien dat insuline resistentie de grootste verklaring gaf voor de associatie tussen het metabool syndroom en ALT. Andere processen die gerelateerd zijn an het metabool syndroom verklaarden ook een deel van de associatie, maar waren niet zo sterk. Ontstekingsmarkers afkomstig uit vetcellen voegden niets toe aan de verklaring gevonden door insuline resistentie $(77 \%)$, terwijl de helft van de verklaring door niet-geësterificeerde vetzuren (NEFA; $5 \%$ wel extra was bovenop insuline resistentie. Dit suggereert dat de helft van de mediatie door NEFA gebeurt volgens routes die samenvallen met processen als ontsteking in het vetweefsel en insuline signalering terwijl de andere helft gebeurt volgens andere processen.

Eerder hebben we al laten zien dat een verhoogde concentratie van complement C3 in het bloed vaak gevonden wordt bij personen met het metabool syndroom. Verschillende aspecten van het metabool syndroom, inclusief insuline resistentie kunnen bijdragen aan verhoogde concentraties van C3 in het bloed. Insuline resistentie is geassocieerd met de concentratie C3 in het bloed in de CODAM populatie, zoals te zien is in hoofdstuk 6 . We hebben tevens laten zien dat nietalcoholische leververvetting, gemeten als ALT, een deel van de associatie kan verklaren, onafhankelijk van obesitas en laaggradige ontsteking.

Met alle bovenstaande bevindingen uit onze onderzoeken laten we zijn dat diverse processen een deel kunnen verklaren van de associatie tussen het metabool syndroom en (metabole intermediairen van) cardiovasculaire ziektes. Met de processen bedoelen 
we laaggradige ontsteking, insuline resistentie, endotheel dysfunctie, complement en niet-geësterificeerde vetzuren. Verder hebben we laten zien dat deze processen deels overlappend zijn en deels onafhankelijk van elkaar. 

Dankwoord 

Eindelijk, het is zover! Het dankwoord kan geschreven worden na vier jaar full-time onderzoek en anderhalf jaar alles afronden in eigen tijd. Misschien verbaast het mezelf nog wel het meest dat het zover gekomen is. Het tot stand komen van dit proefschrift was nooit gelukt zonder de hulp en steun van heel veel mensen.

Als eerste wil ik alle proefpersonen van CODAM bedanken: iedereen die de stap heeft genomen om alle onderzoeken te ondergaan ten tijde van CODAM 1 (1999-2001) en speciaal iedereen die het aandurfde om in de periode 2006-2009 nog een keer terug te komen om twee ochtenden nuchter allerlei onderzoeken te ondergaan voor CODAM 2. Het praktische werk maakte voor mij het promoveren leuk omdat ik met mensen kon werken, verschillende (levens-)verhalen hoorde en niet alleen maar 'achter de computer' hoefde te zitten.

Daarna is mijn promotieteam aan de beurt om bedankt te worden. Promotor Prof. Dr. Stehouwer, beste Coen, bedankt voor al je hulp bij mijn promotie, het lezen van alle artikelen en de opmerkingen die er voor zorgden dat het betere manuscripten werden. Ook wil ik je bedanken voor je steun. In het eerste jaar kwam het bericht dat de externe financiering weg zou vallen en jouw eerste reactie was dat in ieder geval mijn promotietraject veilig was en door moest gaan. Daarvoor ben ik je zeer dankbaar.

Marleen en Carla, mijn co-promotores, ook jullie ben ik veel dank verschuldigd. Jullie hebben mij intensief geholpen om CODAM 2 en ook dit proefschrift tot een succes te brengen. De deur stond altijd open voor al mijn vragen en ook jullie hadden er geen moeite mee om proefpersoon te zijn als er weer eens metingen geoefend moesten worden.

Isabel, je was altijd weer bereidt om een geschreven paper te lezen en van het nodige commentaar te voorzien. Het was niet altijd even leuk om ze van je terug te krijgen, maar de kwaliteit verbeterde er wel door. Verder heb je in het begin veel geholpen door mij te leren hoe ik vaatecho's moet maken, een belangrijk onderdeel van CODAM 2.

CODAM 2 is zo succesvol verlopen dankzij de hulp die ik van velen gekregen heb.

Josephine, jij hebt echt een engelengeduld! De tijd die jij erin gestopt hebt om alle proefpersonen te bellen en te overtuigen om weer aan de onderzoeken mee te doen. Geweldig, zonder jou waren het er veel minder geweest. Dany, ook zonder jouw hulp hadden we niet zo veel personen kunnen includeren. Jij hebt veel voorbereidingswerk gedaan (o.a. stickeren van buisjes) en ook vele metingen heb jij voor je rekening genomen. Eigenlijk moet ik hier iedereen van het lab bedanken, omdat iedereen wel iets heeft gedaan. Vicky en Margee bedankt voor al het pipetteren; Johanna, Lian en 
Roel bedankt voor jullie hulp bij de visites/metingen; Petra en Marjo, bedankt voor het (ont)stickeren van buisjes. Verder is iedereen van de afdeling wel eens of meerdere keren proefpersoon geweest, voor het oefenen en valideren van alle metingen.

Het maken van buikecho's heb jij, Martijn, mij geleerd, dank daarvoor en Frans bedankt voor het beoordelen van de leverecho's. Jos bedankt voor het helpen bij het maken de buikecho's en de IVGTT's en Arne bedankt voor het beoordelen van de ECG's. Jeroen bedankt voor het regelen van de onderzoeksruimte en de houder die ik gebruikte bij de vaatecho's.

Natuurlijk zijn ook de studenten die hun wetenschappelijke stage bij CODAM hebben gedaan belangrijk: Petra, Mieke, Jesse en Mirjam, bedankt voor jullie hulp. En ook de studenten die vele buisjes bloed gepipetteerd hebben, bedankt.

Dan mijn oud-kamergenoten. Steven, we hebben niet heel lang samen op één kamer gezeten, omdat jouw promotie zich in de afrondende fase bevond, maar het was wel een leuke tijd. Katrien we zijn tegelijk begonnen aan onze promotie en Olaf jij ruim een half jaar later. Het waren onderzoeken met verschillende onderwerpen, maar toch kon er genoeg gediscussieerd worden en waren er zeker ook genoeg gezellige gesprekken. Jullie hebben jullie promotie inmiddels allebei al afgerond, super! Veel succes met jullie post-doc onderzoeken. Bas, jij bracht nog een nieuw onderwerp in de kamer: voeding. $\mathrm{Al}$ snel was je aangepast en discussieerde en kletste je gezellig mee in de kamer. Succes verder met jouw promotie, dit geldt ook voor Johanna, Lian, Roel, Marcelle en Nordin.

Ook mijn huidige (oud-)kamergenoten/collega's van de afdeling O\&I van de Inspectie voor de Gezondheidszorg wil ik bedanken voor de interesse die jullie regelmatig toonden in de afronding van mijn promotie.

Tijdens een onderzoeksperiode is ook ontspanning heel erg belangrijk. Ellen, Karin, Anke en Karolien bedankt voor de regelmatige ontzettend gezellige etentjes, uitjes, verjaardagsfeestjes en dergelijke, die zorgden voor een welkome afwisseling. Ellen, nadat je zelf al gepromoveerd bent, mag je nu ook nog meemaken hoe het is om paranimf te zijn. Echt heel leuk dat je deze taak op je wilt nemen. Ook het jaarlijkse ME-weekendje met Karin, Ellen, Cathryn, Karolien, Michiel, Ruud, Anne, Vera, Maud en natuurlijk Egon waren een zeer welkome afwisseling. Helaas kunnen Egon en ik dit jaar zeer waarschijnlijk niet mee, maar volgend jaar zijn we zeker weer van de partij!

Suus en Roy, lieve zus en (schoon)broer, ook jullie hebben voor veel steun, ontspanning en plezier gezorgd. Twee keer per week gingen we gedurende die vier jaar promotieonderzoek samen sporten en konden we gezellig bijkletsen. Afleiding kwam ook door de komst van mijn lieve nichtje Milou en neefje Chris, die er voor zorgden 
dat ik nog vaker bij jullie over de vloer kom. Suus, bedankt dat je mij ook vandaag wilt steunen door mijn paranimf te zijn.

Pap en mam, bedankt voor alles, voor jullie steun, jullie hulp en jullie vertrouwen. Jullie stonden en staan nog steeds altijd voor me klaar en dat is een heerlijk gevoel. En het motto: "het komt allemaal goed", houden we er in.

Egon, we hebben uitgerekend hoeveel kilometers we hebben moeten reizen om tijdens mijn promotietijd in de weekenden bij elkaar te kunnen zijn: $70592 \mathrm{~km}$, oftewel 1.76 keer de aarde rond! Maar het is zeker wel de moeite waard geweest. De weekenden samen zorgden voor de nodige ontspanning in mijn "buitenhuisje" in Amsterdam of in jouw "buitenhuisje" in Meijel. Ook kon ik je bellen als ik weer eens even mijn verhaal kwijt moest. Je was er dag en nacht om me te steunen. Super fijn dat je me geholpen hebt met de laatste zware loodjes van dit proefschrift en nu op naar ons eigen huis, waar we hopelijk snel de sleutel van krijgen en samen heel veel jaren kunnen wonen.

Marjon 



\section{Curriculum Vitae}


Marjon Jacobs werd op 12 augustus 1982 geboren in Meijel. Het VWO diploma behaalde zij in 2000 aan het Bouwens van der Boye College te Panningen, waarna zij begon aan de Bachelor Biomedische Technologie aan de Technische Universiteit Eindhoven waarvan het diploma werd behaald in 2004. De daarop volgende de Master Medical Engineering die werd verzorgd door de Technische Universiteit Eindhoven in combinatie met het Academisch Ziekenhuis Maastricht / Universiteit Maastricht. Een stage werd afgerond op de afdeling radiologie en het afstudeeronderzoek werd verricht bij het radiotherapeutisch instituut MAASTRO waarvan ze de resultaten mocht presenteren op het ESTRO congres te Lissabon en later ook werden gepubliceerd. In de zomer van 2005 behaalde ze het Masterdiploma. Vanaf januari 2006 werkte zij gedurende vier jaar aan haar promotieonderzoek bij het laboratorium voor metabolisme en vasculaire geneeskunde van de Universiteit Maastricht, waarvan de resultaten zijn beschreven in dit proefschrift. Gedurende haar onderzoeksperiode bezocht zij congressen over diabetes in Amsterdam (Nederland), Rome (Italië) en Wenen (Oostenrijk). Sinds april 2010 werkt zij als stafmedewerker op de afdeling Onderzoek en Innovatie van de Inspectie voor de Gezondheidszorg. 
Publications 
Jacobs M, van Greevenbroek MM, van der Kallen CJ, et al. Low-grade inflammation can partly explain the association between the metabolic syndrome and either coronary artery disease or severity of peripheral arterial disease: the CODAM study. Eur J Clin Invest 2009;39(6):437-444.

Jacobs M, van Greevenbroek MM, van der Kallen CJ, et al. The association between the metabolic syndrome and peripheral, but not coronary, artery disease is partly mediated by endothelial dysfunction: the CODAM study. Eur J Clin Invest 2011;41(2):167-175.

Van Greevenbroek MM, Jacobs M, van der Kallen CJ, et al. Human plasma complement C3 is independently associated with coronary heart disease, but only in heavy smokers (the CODAM study). Int J Cardiol ( doi:10.1016/j.ijcard.2010.09.017).

Jacobs M, van Greevenbroek MM, van der Kallen CJ, et al. The association between the metabolic syndrome and alanine amino transferase is mediated by insulin resistance via related metabolic intermediates (the Cohort on Diabetes and Atherosclerosis Maastricht [CODAM] study). Metabolism 2011;60(7):969-975.

Van Greevenbroek MM, Jacobs M, Van Der Kallen CJ, et al. The cross-sectional association between insulin resistance and circulating complement C3 is partly explained by plasma alanine aminotransferase, independent of central obesity and general inflammation (the CODAM study). Eur J Clin Invest 2011;41(4):372-379.

Nijsten SM, van Elmpt WJ, Jacobs M, et al. A global calibration model for a-Si EPIDs used for transit dosimetry. Med Phys 2007;34(10):3872-3884. 


\section{Abbreviations}




\begin{tabular}{|c|c|}
\hline AAIx & ankle-arm index \\
\hline AHA & American Health Association \\
\hline ALT & alanine aminotransferase \\
\hline CAD & coronary artery disease \\
\hline $\mathrm{CHD}$ & coronary heart disease \\
\hline CODAM & Cohort on Diabetes and Atherosclerosis Maastricht \\
\hline CRP & C-reactive protein \\
\hline CVD & cardiovascular disease \\
\hline ECG & electrocardiogram \\
\hline EGIR & European Group for Study of Insulin Resistance \\
\hline HDL & high-density lipoprotein \\
\hline ICAM & intercellular adhesion molecule \\
\hline IDF & International Diabetes Federation \\
\hline IFG & impaired fasting glucose \\
\hline IGM & impaired glucose metabolism \\
\hline IGT & impaired glucose tolerance \\
\hline IL & interleukin \\
\hline IRS & insulin receptor substrate \\
\hline LDL & low-density lipoprotein \\
\hline MCP & monocyte chemoattractant protein \\
\hline NAFLD & non-alcoholic fatty liver disease \\
\hline NCEP-ATP III & $\begin{array}{l}\text { National Cholesterol Education Program Adult Treatment } \\
\text { Panel III }\end{array}$ \\
\hline NEFA & non-esterified fatty acids \\
\hline NGT & normal glucose tolerance \\
\hline NHLBI & National Heart Lung and Blood Institute \\
\hline OGTT & oral glucose tolerance test \\
\hline PAD & peripheral arterial disease \\
\hline PAI & plasminogen activator inhibitor \\
\hline PKC & protein kinase $\mathrm{C}$ \\
\hline SAA & serum amyloid A \\
\hline T2DM & type 2 diabetes mellitus \\
\hline $\mathrm{TNF} \alpha$ & tumour necrosis factor $\alpha$ \\
\hline VCAM & vascular cell adhesion molecule \\
\hline VLDL & very-low-density lipoprotein \\
\hline vWF & von Willebrand factor \\
\hline WHO & World Health Organisation \\
\hline
\end{tabular}



